\title{
Construction of gem-Difluoroenol Esters through Catalytic $O$-Selective Addition of Difluoroenoxysilanes to Ketenes
}

\author{
Yang Shi, ${ }^{a}$ Bo-Wen Pan, ${ }^{* a}$ Jun-Xiong He, ${ }^{\mathrm{b}}$ Ying Zhou, ${ }^{* a}$ Jian Zhou, ${ }^{\mathrm{b}, \mathrm{c}}$ and Jin-Sheng $\mathrm{Yu}^{* \mathrm{~b}}$ \\ ${ }^{a}$ College of Pharmacy, Guizhou University of Traditional Chinese Medicine, Guiyang 550025, China. \\ ${ }^{b}$ Shanghai Engineering Research Center of Molecular Therapeutics and New Drug Development, and Shanghai Key \\ Laboratory of Green Chemistry and Chemical Processes, East China Normal University, Shanghai 200062, China. \\ ${ }^{c}$ State Key Laboratory of Organometallic Chemistry, Shanghai Institute of Organic Chemistry, Shanghai 200032, China. \\ E-mail: bwpan@pku.edu.cn for B.-W.P.; yingzhou71@126.com for Y.Z.; jsyu@ @chem.ecnu.edu.cn for J.-S.Y.
}

\begin{tabular}{|l|c|}
\hline \multicolumn{1}{|c|}{ Table of Contents } & Page \\
\hline 1. X-ray crystallographic data of $\mathbf{3 b}$ & S2-S6 \\
\hline $2 .{ }^{1} \mathrm{H},{ }^{13} \mathrm{C}$, and ${ }^{19} \mathrm{~F}$ NMR spectra & S7-S81 \\
\hline
\end{tabular}




\section{X-ray crystallographic data of $3 b^{1}$}

Data intensity of 3b was collected using a 'XtaLAB AFC12 (RINC)' diffractometer at 169.99(10) K. Data collection and reduction were done by using Olex2 and the structure was solved with the ShelXT structure solution program using Intrinsic Phasing and refined with the XL refinement package using Least Squares minimisation. Crystal data for $\mathbf{3 b}$ : $\mathrm{C}_{18} \mathrm{H}_{15} \mathrm{ClF}_{2} \mathrm{O}_{2}, T=169.99(10) \mathrm{K}$, triclinic, $\mathrm{P}-1, a=5.6942(2) \AA, b=7.8143(2) \AA, c=18.9954(4) \AA, \alpha=78.225(2)^{\circ}, \beta=84.514(2)^{\circ}, \gamma=$ $86.118(2)^{\circ}, V=822.64(4) \AA^{3} . \mathrm{Z}=2, \rho_{\text {calc }}=1.359 \mathrm{~g} / \mathrm{cm}^{3} .7847$ reflections collected, $2898\left[\mathrm{R}_{\text {int }}=\right.$ $\left.0.0304, \mathrm{R}_{\text {sigma }}=0.0292\right]$ independent reflections, $\mathrm{R}_{1}=0.0410, \mathrm{wR}_{2}=0.1039(I>2 \sigma(I)$, final $), \mathrm{R}_{1}=$ $0.0434, \mathrm{wR}_{2}=0.1060$ (all data), $\mathrm{GOF}=1.039$, and 209 parameters.

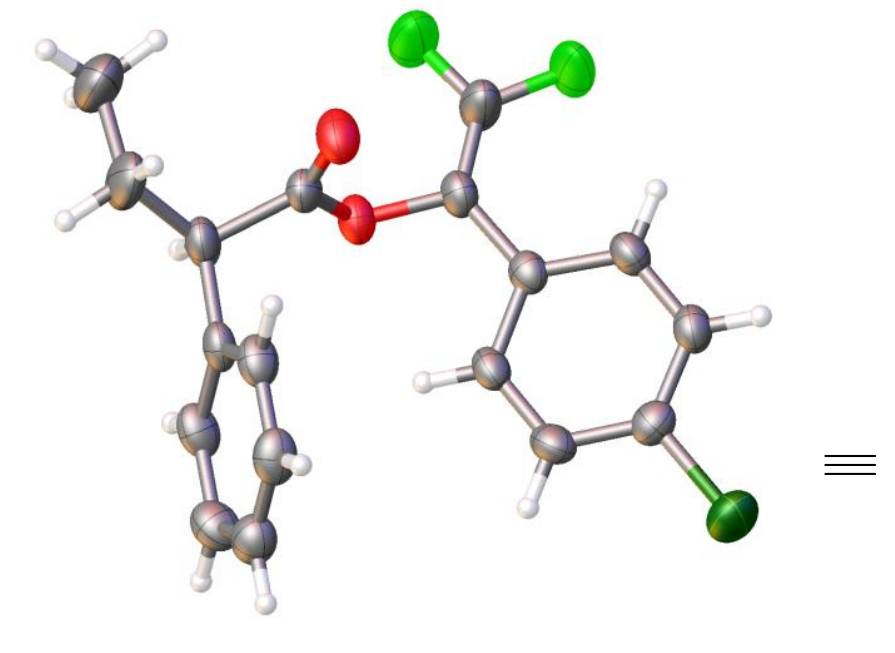

X-Ray of $3 \mathbf{b}$ (ORTEP, ellipsoids set at $50 \%$ probability)<smiles>CCC(C(=O)OC(=C(F)F)c1ccc(Cl)cc1)c1ccccc1</smiles>

3b

Table S1. Crystal data and structure refinement for $\mathbf{3 b}$.

Identification code

Empirical formula

Formula weight

Temperature/K

Crystal system

Space group

a/A

b/A

c/Å 3b

$\mathrm{C}_{18} \mathrm{H}_{15} \mathrm{ClF}_{2} \mathrm{O}_{2}$

336.75

169.99(10)

triclinic

P-1

5.6942(2)

7.8143(2)

18.9954(4)

1 Supplementary crystallographic data have been deposited at Cambridge Crystallographic Data Center (CCDC number: 2060148). 


\begin{tabular}{|c|c|}
\hline$\alpha /{ }^{\circ}$ & $78.225(2)$ \\
\hline$\beta /{ }^{\circ}$ & $84.514(2)$ \\
\hline$\gamma /{ }^{\circ}$ & $86.118(2)$ \\
\hline Volume $/ \AA^{3}$ & $822.64(4)$ \\
\hline $\mathrm{Z}$ & 2 \\
\hline$\rho_{\text {calc }} \mathrm{g} / \mathrm{cm}^{3}$ & 1.359 \\
\hline$\mu / \mathrm{mm}^{-1}$ & 2.303 \\
\hline $\mathrm{F}(000)$ & 348.0 \\
\hline Crystal size $/ \mathrm{mm}^{3}$ & $0.42 \times 0.38 \times 0.36$ \\
\hline Radiation & $\mathrm{CuK} \alpha(\lambda=1.54184)$ \\
\hline $2 \Theta$ range for data collection ${ }^{\circ}$ & 9.546 to 134.12 \\
\hline Index ranges & $-6 \leq \mathrm{h} \leq 6,-9 \leq \mathrm{k} \leq 9,-14 \leq 1 \leq 22$ \\
\hline Reflections collected & 7847 \\
\hline Independent reflections & $2898\left[\mathrm{R}_{\mathrm{int}}=0.0304, \mathrm{R}_{\text {sigma }}=0.0292\right]$ \\
\hline Data/restraints/parameters & 2898/0/209 \\
\hline Goodness-of-fit on $\mathrm{F}^{2}$ & 1.039 \\
\hline Final $R$ indexes $[I>=2 \sigma(I)]$ & $\mathrm{R}_{1}=0.0410, \mathrm{wR}_{2}=0.1039$ \\
\hline Final $\mathrm{R}$ indexes [all data] & $\mathrm{R}_{1}=0.0434, w \mathrm{R}_{2}=0.1060$ \\
\hline Largest diff. peak/hole / e $\AA^{-3}$ & $0.25 /-0.27$ \\
\hline
\end{tabular}

Table S2. Fractional Atomic Coordinates $\left(\times 10^{4}\right)$ and Equivalent Isotropic Displacement Parameters $\left(\AA^{2} \times 10^{3}\right)$ for $3 \mathbf{b} . \mathrm{U}_{\text {eq }}$ is defined as $1 / 3$ of of the trace of the orthogonalised $U_{\text {IJ }}$ tensor.

$\begin{array}{lllll}\text { Atom } & \boldsymbol{x} & \boldsymbol{y} & \boldsymbol{z} & \mathbf{U}(\mathbf{e q}) \\ \mathrm{C} 1 & 3106(3) & 8134(2) & 7029.6(9) & 41.3(4) \\ \mathrm{C} 2 & 3403(3) & 8608(2) & 6286.8(10) & 44.9(4) \\ \text { C3 } & 5459(3) & 8120(2) & 5916.7(10) & 46.5(4) \\ \text { C4 } & 7213(3) & 7161(2) & 6295.9(10) & 47.8(4) \\ \text { C5 } & 6925(3) & 6683(2) & 7035.6(10) & 42.6(4) \\ \text { C6 } & 4874(3) & 7148.5(18) & 7417.4(9) & 36.5(3) \\ \text { C7 } & 4572(3) & 6558(2) & 8231.9(9) & 43.0(4) \\ \text { C8 } & 2454(3) & 5431(2) & 8401.2(8) & 36.5(3) \\ \text { C9 } & 1256(3) & 2681(2) & 8309.2(9) & 39.3(4) \\ \text { C10 } & 439(4) & 1830(3) & 8941.5(10) & 59.8(5) \\ \text { C11 } & 577(3) & 2586.2(18) & 7594.0(8) & 33.2(3) \\ \text { C12 } & -1452(3) & 1772(2) & 7496.7(9) & 38.7(3) \\ \text { C13 } & -2053(3) & 1722(2) & 6818.3(9) & 40.1(4) \\ \text { C14 } & -653(3) & 2515(2) & 6223.6(9) & 39.9(4)\end{array}$




$\begin{array}{lllll}\text { C15 } & 1354(3) & 3333(2) & 6297.6(9) & 40.9(4) \\ \text { C16 } & 1961(3) & 3363.6(19) & 6980.8(8) & 36.0(3) \\ \text { C17 } & 4271(5) & 8071(3) & 8631.9(11) & 69.3(6) \\ \text { C18 } & 4330(5) & 7480(4) & 9443.8(12) & 85.4(8) \\ \text { C11 } & -1467.3(10) & 2491.1(7) & 5366.3(2) & 64.68(18) \\ \text { F1 } & 1139(3) & 2006(2) & 9561.1(6) & 84.0(4) \\ \text { F2 } & -1203(3) & 659.5(18) & 9051.6(6) & 86.3(5) \\ \text { O1 } & 465(2) & 5885.3(16) & 8553.1(7) & 51.7(3) \\ \text { O2 } & 3118.9(18) & 3766.1(14) & 8318.0(6) & 39.6(3)\end{array}$

Table S3. Anisotropic Displacement Parameters $\left(\AA^{2} \times 10^{3}\right)$ for $\mathbf{3 b}$. The Anisotropic displacement factor exponent takes the form: $-2 \pi^{2}\left[h^{2} a^{* 2} U_{11}+2 h k a * b * U_{12}+\ldots\right]$.

$\begin{array}{lllllll}\text { Atom } & \mathbf{U}_{11} & \mathbf{U}_{\mathbf{2 2}} & \mathbf{U}_{\mathbf{3 3}} & \mathbf{U} \mathbf{2 3} & \mathbf{U} 13 & \mathbf{U}_{\mathbf{1 2}} \\ \text { C1 } & 31.7(8) & 36.3(8) & 57.9(10) & -12.8(7) & -7.0(7) & -1.0(6) \\ \text { C2 } & 43.3(9) & 37.1(8) & 55.0(10) & -4.7(7) & -18.5(7) & 0.1(7) \\ \text { C3 } & 51.4(10) & 42.6(9) & 46.2(9) & -6.9(7) & -7.0(7) & -8.2(7) \\ \text { C4 } & 39.3(9) & 45.2(9) & 57.1(10) & -9.3(8) & 2.8(7) & -1.8(7) \\ \text { C5 } & 31.0(8) & 37.1(8) & 58.1(10) & -2.8(7) & -9.3(7) & -2.5(6) \\ \text { C6 } & 33.1(8) & 31.2(7) & 46.9(9) & -6.5(6) & -8.6(6) & -10.6(6) \\ \text { C7 } & 38.9(9) & 44.3(9) & 48.0(9) & -7.3(7) & -9.3(7) & -16.8(7) \\ \text { C8 } & 33.7(8) & 41.6(8) & 36.5(8) & -9.6(6) & -5.1(6) & -10.8(6) \\ \text { C9 } & 40.7(8) & 35.3(8) & 43.5(9) & -8.1(6) & -2.4(6) & -13.3(6) \\ \text { C10 } & 80.0(14) & 62.4(11) & 40.6(10) & -6.8(8) & -6.5(9) & -38.0(10) \\ \text { C11 } & 32.6(7) & 26.9(7) & 40.3(8) & -8.2(6) & -0.6(6) & -3.3(6) \\ \text { C12 } & 35.3(8) & 37.8(8) & 42.3(8) & -6.0(6) & 3.1(6) & -11.6(6) \\ \text { C13 } & 34.3(8) & 39.6(8) & 48.8(9) & -12.4(7) & -3.4(7) & -8.2(6) \\ \text { C14 } & 44.0(9) & 39.3(8) & 38.6(8) & -13.1(6) & -4.0(7) & -1.7(7) \\ \text { C15 } & 39.4(8) & 42.5(8) & 39.9(8) & -9.8(7) & 8.8(6) & -7.3(7) \\ \text { C16 } & 29.0(7) & 34.9(7) & 45.5(9) & -12.5(6) & 3.9(6) & -6.8(6) \\ \text { C17 } & 96.5(17) & 60.6(12) & 60.5(12) & -23.2(10) & -4.4(11) & -42.1(12) \\ \text { C18 } & 109(2) & 102.9(19) & 57.4(13) & -32.4(13) & -5.8(13) & -47.1(16) \\ \text { C11 } & 79.5(4) & 78.3(4) & 40.8(3) & -14.2(2) & -10.0(2) & -22.6(3) \\ \text { F1 } & 114.8(11) & 102.2(10) & 39.1(6) & -3.0(6) & -13.1(6) & -59.1(8) \\ \text { F2 } & 122.5(12) & 94.3(9) & 44.7(6) & 1.5(6) & -1.9(7) & -78.6(9) \\ \text { O1 } & 39.8(7) & 51.6(7) & 69.0(8) & -24.0(6) & -0.3(6) & -8.3(5) \\ \text { O2 } & 35.7(6) & 36.7(6) & 48.4(6) & -9.6(5) & -6.1(4) & -9.0(4)\end{array}$


Table S4. Bond Lengths for $\mathbf{3 b}$.

\begin{tabular}{|c|c|c|c|c|c|}
\hline Atom & Atom & Length/Å & Atom & Atom & Length/Å \\
\hline $\mathrm{C} 1$ & $\mathrm{C} 2$ & $1.381(2)$ & C9 & $\mathrm{C} 11$ & $1.465(2)$ \\
\hline $\mathrm{C} 1$ & C6 & $1.398(2)$ & C9 & $\mathrm{O} 2$ & $1.4052(18)$ \\
\hline $\mathrm{C} 2$ & $\mathrm{C} 3$ & $1.379(3)$ & $\mathrm{C} 10$ & F1 & $1.314(2)$ \\
\hline $\mathrm{C} 3$ & $\mathrm{C} 4$ & $1.376(3)$ & $\mathrm{C} 10$ & $\mathrm{~F} 2$ & $1.325(2)$ \\
\hline $\mathrm{C} 4$ & $\mathrm{C} 5$ & $1.375(3)$ & C11 & $\mathrm{C} 12$ & $1.401(2)$ \\
\hline $\mathrm{C} 5$ & $\mathrm{C} 6$ & $1.382(2)$ & C11 & $\mathrm{C} 16$ & $1.395(2)$ \\
\hline C6 & C7 & $1.518(2)$ & $\mathrm{C} 12$ & $\mathrm{C} 13$ & $1.373(2)$ \\
\hline $\mathrm{C} 7$ & $\mathrm{C} 8$ & $1.512(2)$ & $\mathrm{C} 13$ & C14 & $1.380(2)$ \\
\hline $\mathrm{C} 7$ & $\mathrm{C} 17$ & $1.523(3)$ & C14 & $\mathrm{C} 15$ & $1.379(2)$ \\
\hline $\mathrm{C} 8$ & $\mathrm{O} 1$ & $1.1909(19)$ & C14 & $\mathrm{Cl} 1$ & $1.7392(16)$ \\
\hline $\mathrm{C} 8$ & $\mathrm{O} 2$ & $1.3658(19)$ & C15 & C16 & $1.380(2)$ \\
\hline C9 & $\mathrm{C} 10$ & $1.308(3)$ & $\mathrm{C} 17$ & $\mathrm{C} 18$ & $1.520(3)$ \\
\hline
\end{tabular}

Table S5. Bond Angles for 3b.

\begin{tabular}{|c|c|c|c|c|c|c|c|}
\hline Atom & Atom & Atom & Angle $/^{\circ}$ & Atom & Atom & Atom & Angle $/^{\circ}$ \\
\hline $\mathrm{C} 2$ & $\mathrm{C} 1$ & C6 & $120.66(15)$ & $\mathrm{O} 2$ & C9 & $\mathrm{C} 11$ & $115.84(13)$ \\
\hline $\mathrm{C} 3$ & $\mathrm{C} 2$ & $\mathrm{C} 1$ & $120.31(15)$ & C9 & $\mathrm{C} 10$ & F1 & $124.84(16)$ \\
\hline $\mathrm{C} 4$ & $\mathrm{C} 3$ & $\mathrm{C} 2$ & $119.29(17)$ & C9 & $\mathrm{C} 10$ & $\mathrm{~F} 2$ & $125.11(16)$ \\
\hline $\mathrm{C} 5$ & $\mathrm{C} 4$ & $\mathrm{C} 3$ & $120.64(16)$ & F1 & $\mathrm{C} 10$ & $\mathrm{~F} 2$ & $110.05(15)$ \\
\hline $\mathrm{C} 4$ & $\mathrm{C} 5$ & C6 & $121.08(15)$ & $\mathrm{C} 12$ & $\mathrm{C} 11$ & C9 & $122.54(14)$ \\
\hline $\mathrm{C} 1$ & C6 & $\mathrm{C} 7$ & $121.98(15)$ & C16 & $\mathrm{C} 11$ & C9 & $119.38(13)$ \\
\hline $\mathrm{C} 5$ & C6 & $\mathrm{C} 1$ & $118.01(15)$ & C16 & $\mathrm{C} 11$ & $\mathrm{C} 12$ & $118.07(14)$ \\
\hline $\mathrm{C} 5$ & C6 & $\mathrm{C} 7$ & $119.99(14)$ & $\mathrm{C} 13$ & $\mathrm{C} 12$ & $\mathrm{C} 11$ & $121.10(14)$ \\
\hline C6 & $\mathrm{C} 7$ & $\mathrm{C} 17$ & $113.30(15)$ & $\mathrm{C} 12$ & $\mathrm{C} 13$ & C14 & $119.29(14)$ \\
\hline $\mathrm{C} 8$ & C7 & C6 & $106.90(12)$ & $\mathrm{C} 13$ & $\mathrm{C} 14$ & $\mathrm{Cl} 1$ & $118.97(12)$ \\
\hline $\mathrm{C} 8$ & $\mathrm{C} 7$ & $\mathrm{C} 17$ & $111.07(15)$ & C15 & $\mathrm{C} 14$ & $\mathrm{C} 13$ & $121.29(15)$ \\
\hline $\mathrm{O} 1$ & $\mathrm{C} 8$ & $\mathrm{C} 7$ & $127.17(15)$ & C15 & $\mathrm{C} 14$ & $\mathrm{Cl} 1$ & $119.73(13)$ \\
\hline $\mathrm{O} 1$ & $\mathrm{C} 8$ & $\mathrm{O} 2$ & $123.09(14)$ & C14 & $\mathrm{C} 15$ & $\mathrm{C} 16$ & $119.13(14)$ \\
\hline $\mathrm{O} 2$ & $\mathrm{C} 8$ & $\mathrm{C} 7$ & $109.66(13)$ & $\mathrm{C} 15$ & $\mathrm{C} 16$ & $\mathrm{C} 11$ & $121.11(14)$ \\
\hline $\mathrm{C} 10$ & C9 & C11 & $128.79(15)$ & $\mathrm{C} 18$ & $\mathrm{C} 17$ & $\mathrm{C} 7$ & $113.05(19)$ \\
\hline $\mathrm{C} 10$ & C9 & $\mathrm{O} 2$ & $115.29(15)$ & $\mathrm{C} 8$ & $\mathrm{O} 2$ & C9 & $115.38(12)$ \\
\hline
\end{tabular}

Table S6. Hydrogen Atom Coordinates $\left(\AA \times 10^{4}\right)$ and Isotropic Displacement Parameters $\left(\AA^{2} \times 10^{3}\right)$ for $3 b$. 


$\begin{array}{lllll}\text { Atom } & \boldsymbol{x} & \boldsymbol{y} & \boldsymbol{z} & \mathbf{U}(\mathbf{e q}) \\ \text { H1 } & 1716.58 & 8474.76 & 7274.38 & 50 \\ \text { H2 } & 2210.87 & 9258.65 & 6034.74 & 54 \\ \text { H3 } & 5658.51 & 8435.07 & 5416.26 & 56 \\ \text { H4 } & 8606.67 & 6832.74 & 6049.46 & 57 \\ \text { H5 } & 8130.28 & 6036.54 & 7283.05 & 51 \\ \text { H7 } & 5973.47 & 5833.46 & 8388.14 & 52 \\ \text { H12 } & -2408.44 & 1257.62 & 7898.1 & 46 \\ \text { H13 } & -3387.96 & 1158.61 & 6760.28 & 48 \\ \text { H15 } & 2286.01 & 3857.83 & 5892.4 & 49 \\ \text { H16 } & 3315.18 & 3911.5 & 7033.46 & 43 \\ \text { H17A } & 5519.98 & 8871.93 & 8452.12 & 83 \\ \text { H17B } & 2776.26 & 8702.39 & 8530.19 & 83 \\ \text { H18A } & 5772.49 & 6805.09 & 9547.94 & 128 \\ \text { H18B } & 3008.98 & 6775.67 & 9632.36 & 128 \\ \text { H18C } & 4247.08 & 8486.99 & 9664.07 & 128\end{array}$




\section{2. ${ }^{1} \mathrm{H},{ }^{13} \mathrm{C}$, and ${ }^{19} \mathrm{~F}$ NMR spectra}

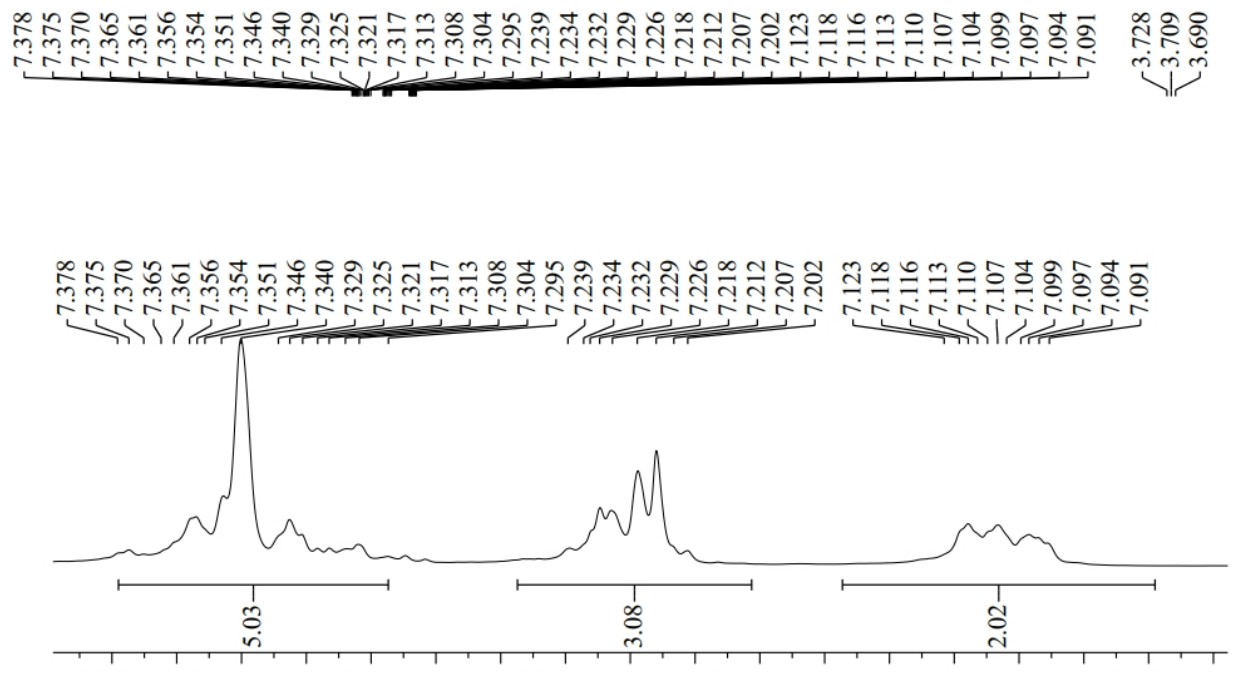

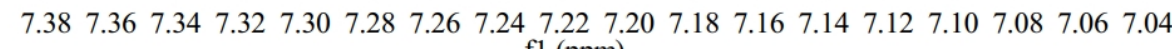
f1 (ppm)
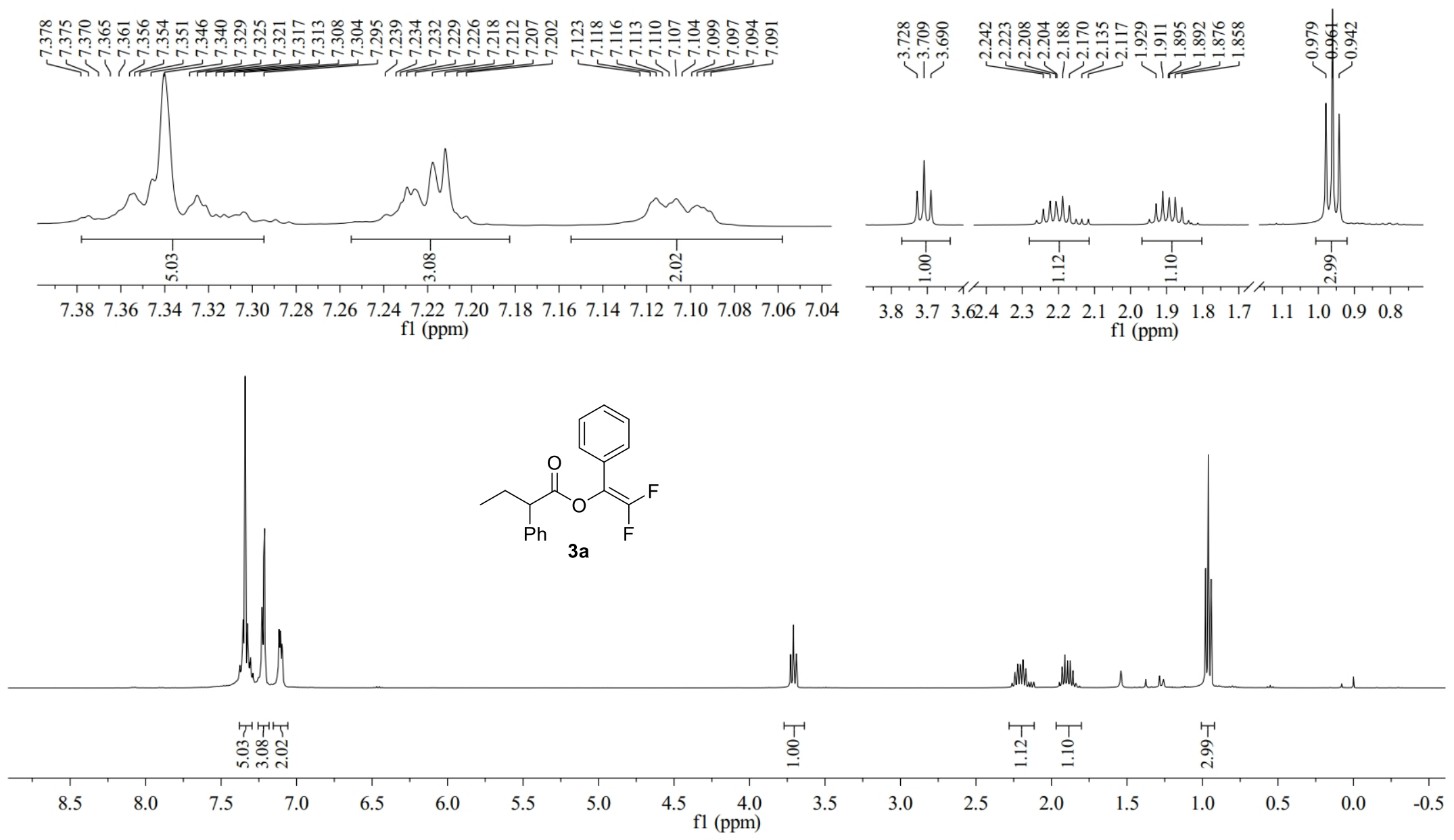

${ }^{1} \mathrm{H}$ NMR of Compound $\mathbf{3 a}\left(400 \mathrm{MHz}, \mathrm{CDCl}_{3}\right)$ 

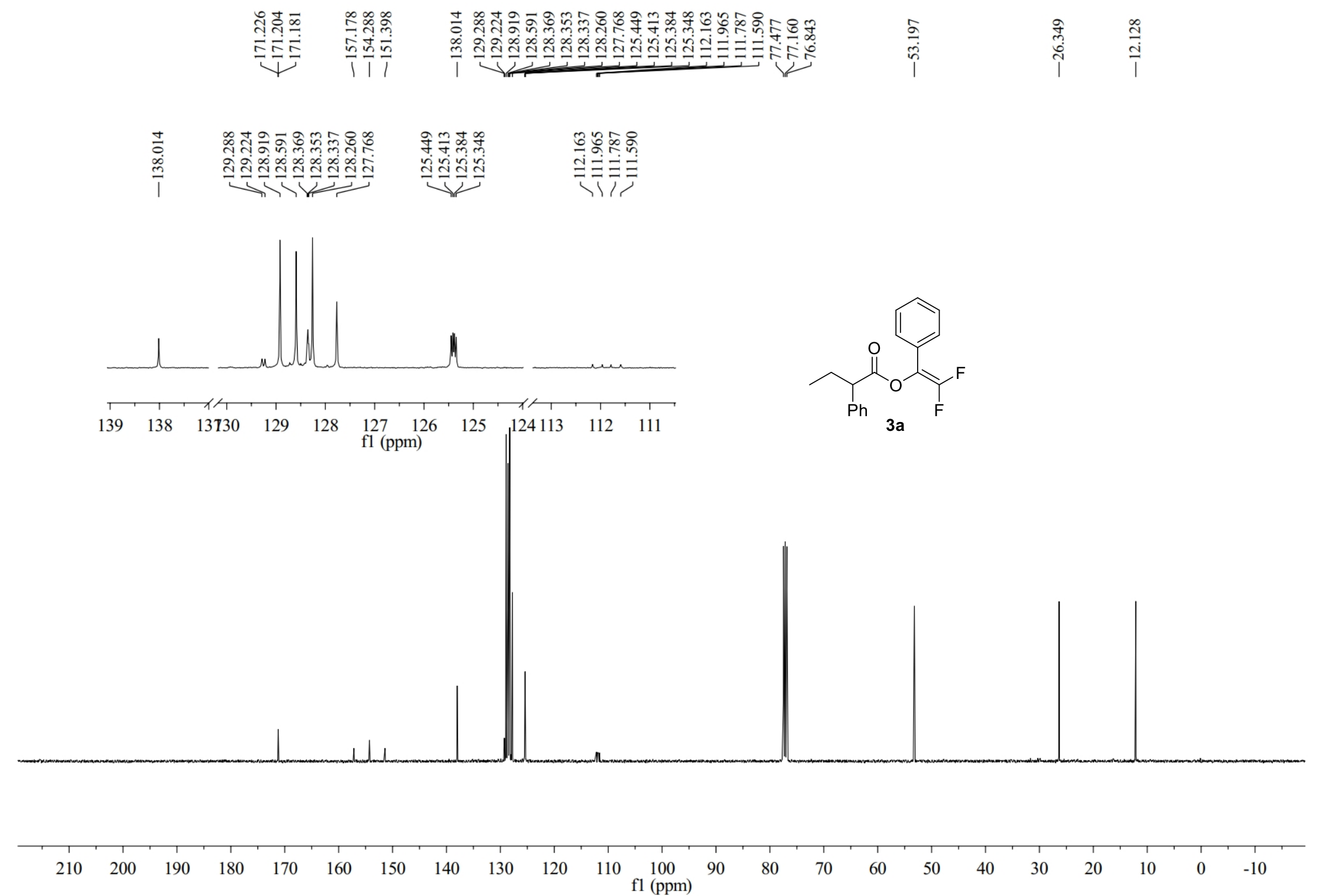

${ }^{13} \mathrm{C}$ NMR of Compound $\mathbf{3 a}\left(100 \mathrm{MHz}, \mathrm{CDCl}_{3}\right)$ 


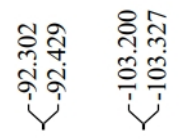

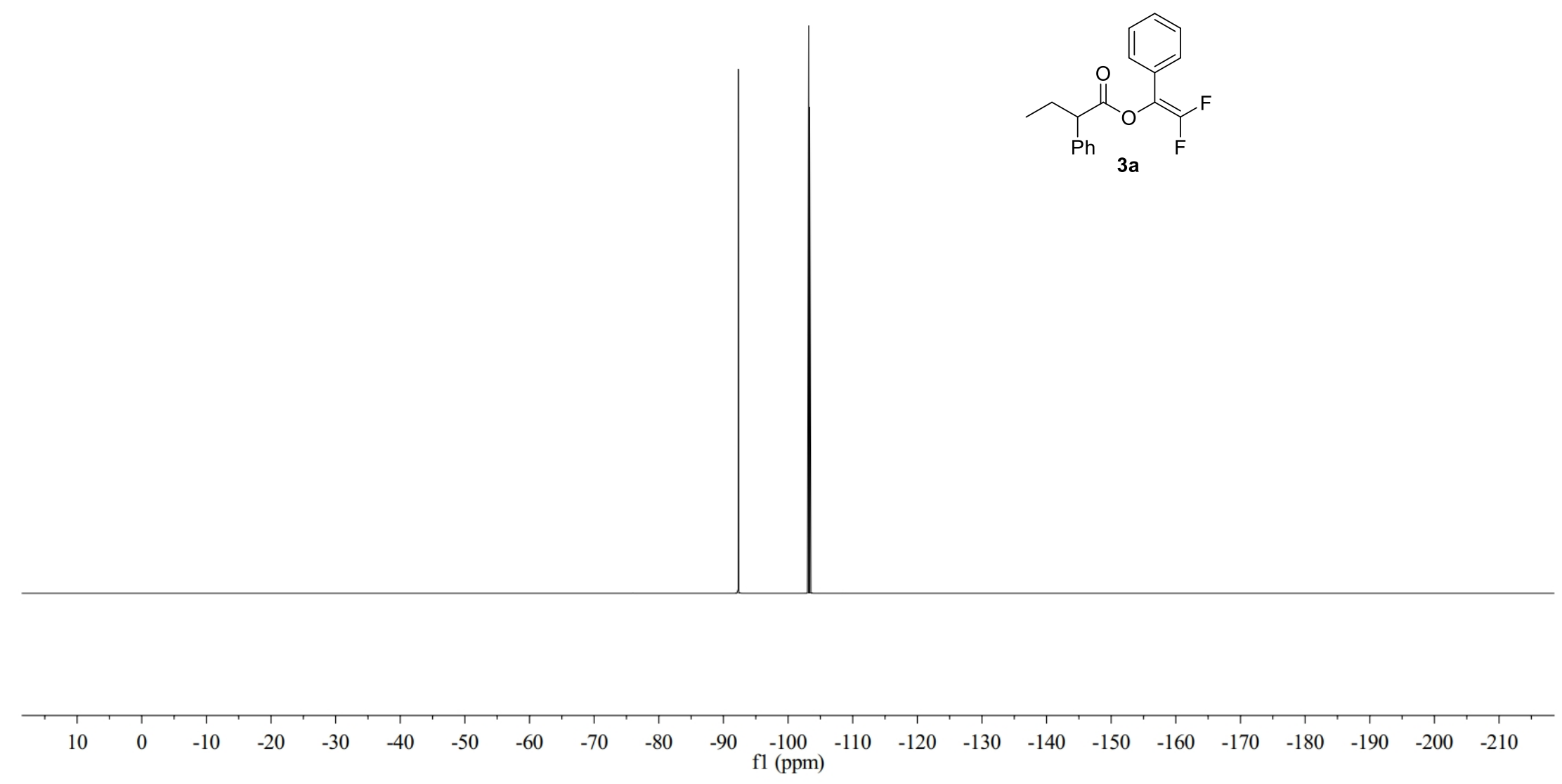

${ }^{19} \mathrm{~F}$ NMR of Compound 3a (376 MHz, $\left.\mathrm{CDCl}_{3}\right)$ 

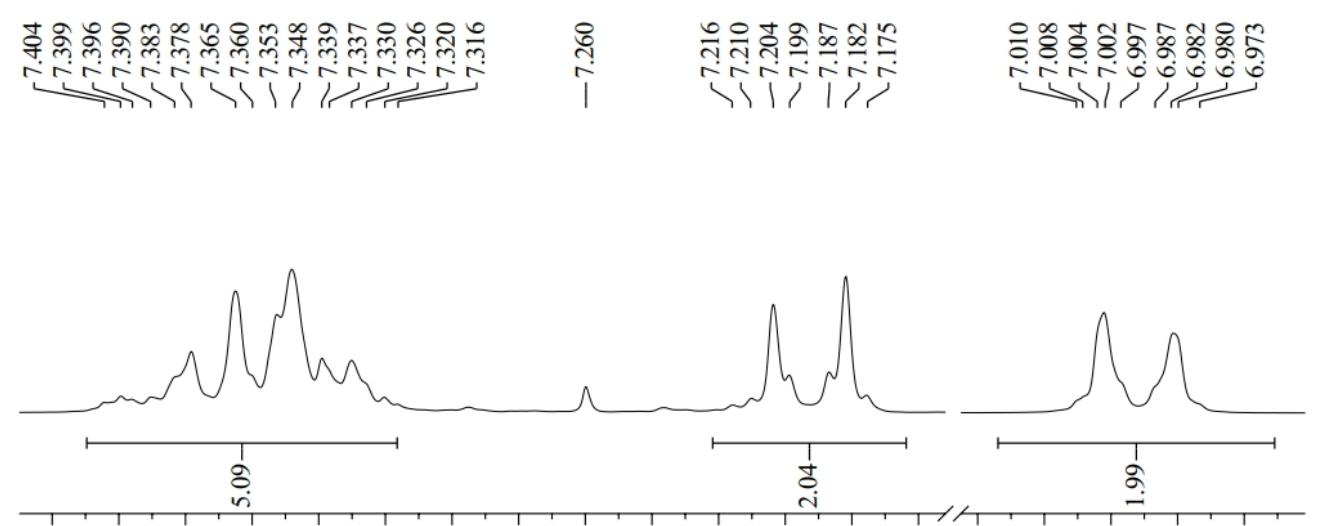

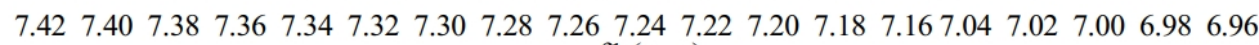

f1 (ppm)
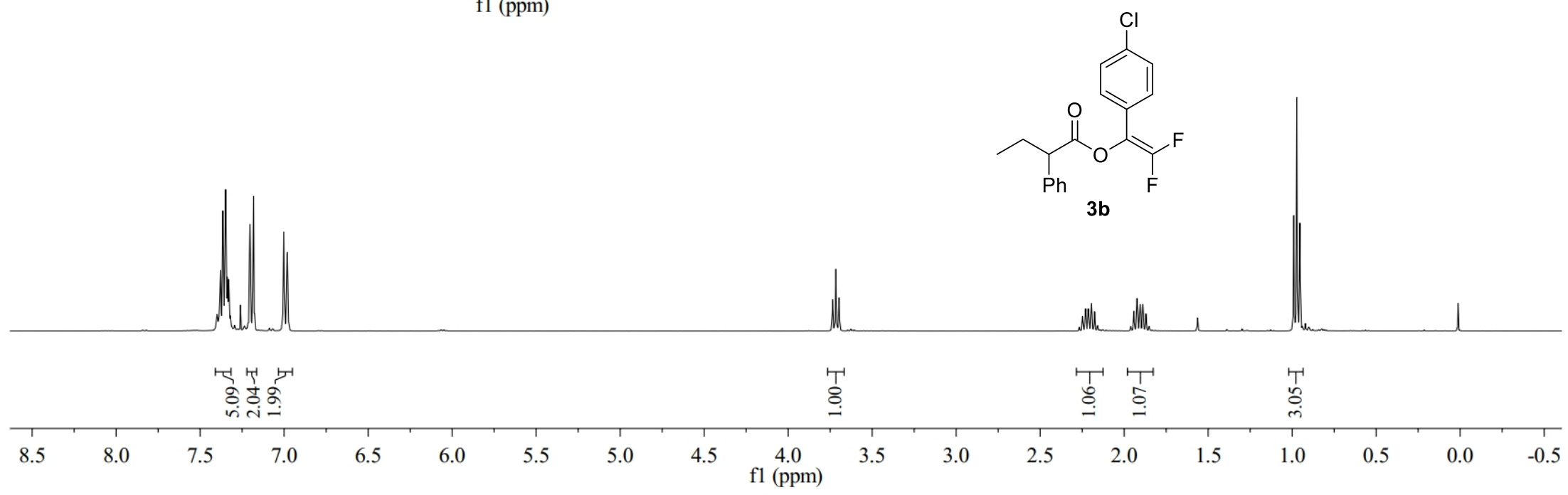

${ }^{1} \mathrm{H}$ NMR of Compound $\mathbf{3 b}\left(400 \mathrm{MHz}, \mathrm{CDCl}_{3}\right)$ 


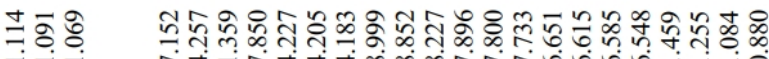

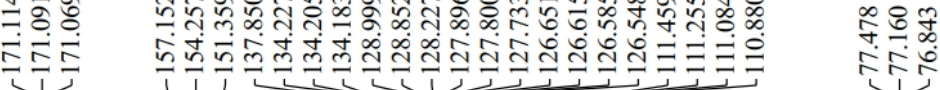

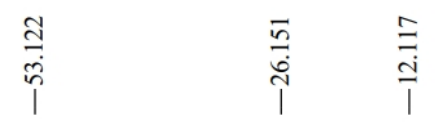

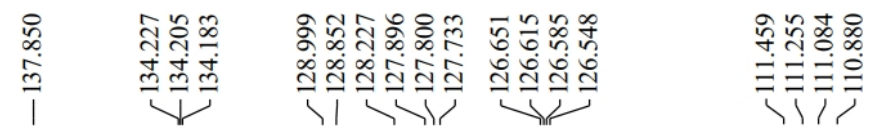
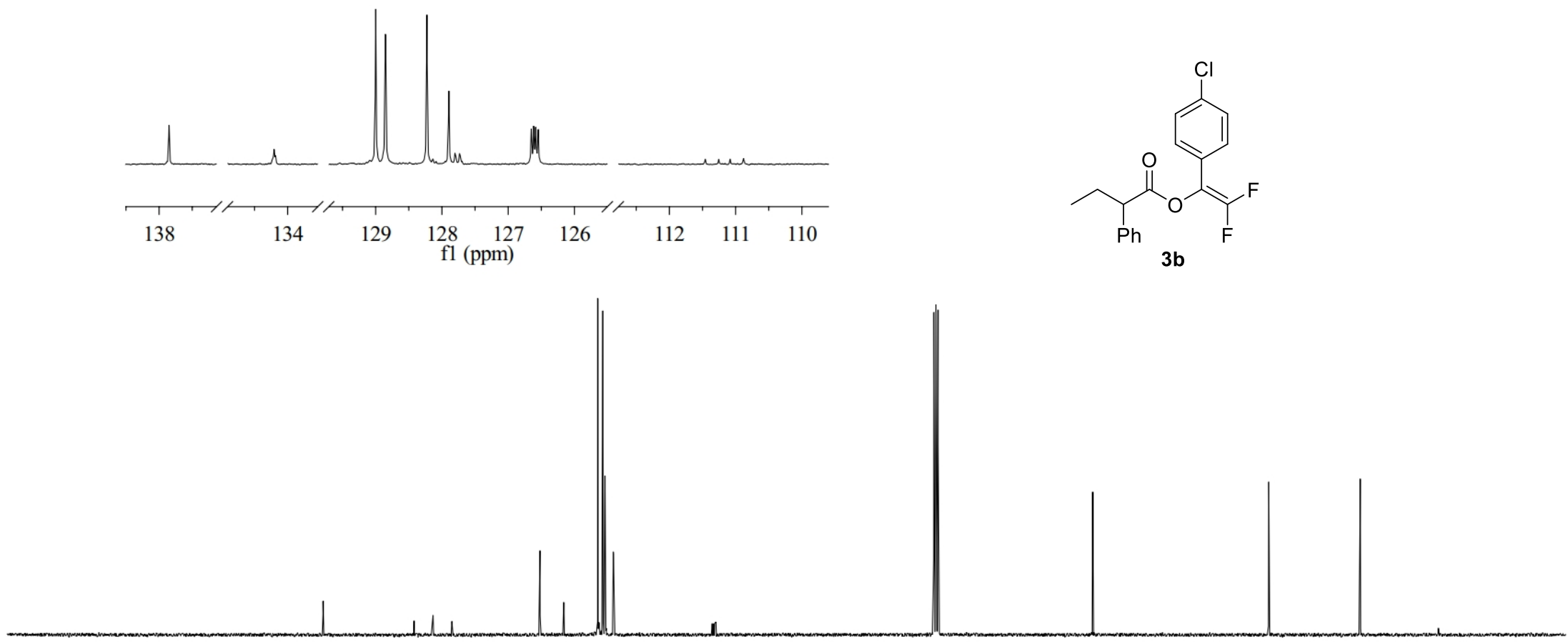

$200 \quad 190$

$180 \quad 170$

160150

140

130

$\begin{array}{llc}120 & 110 & 100 \\ \mathrm{fl}(\mathrm{ppm})\end{array}$

80

$70 \quad 60$

$60 \quad 50$

40

$30 \quad 20$

10

${ }^{13} \mathrm{C}$ NMR of Compound 3b (100 MHz, $\left.\mathrm{CDCl}_{3}\right)$ 


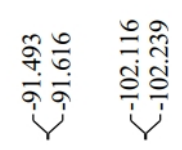

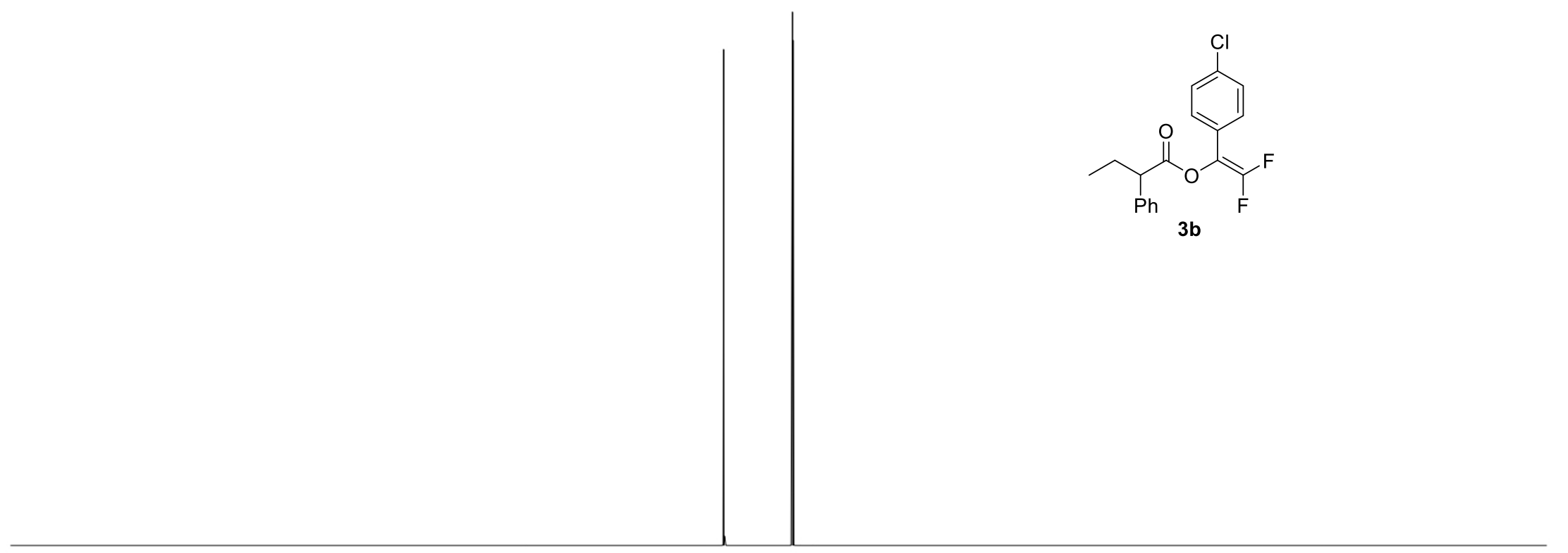
fl (ppm)

${ }^{19} \mathrm{~F}$ NMR of Compound 3b $\left(376 \mathrm{MHz}, \mathrm{CDCl}_{3}\right)$ 

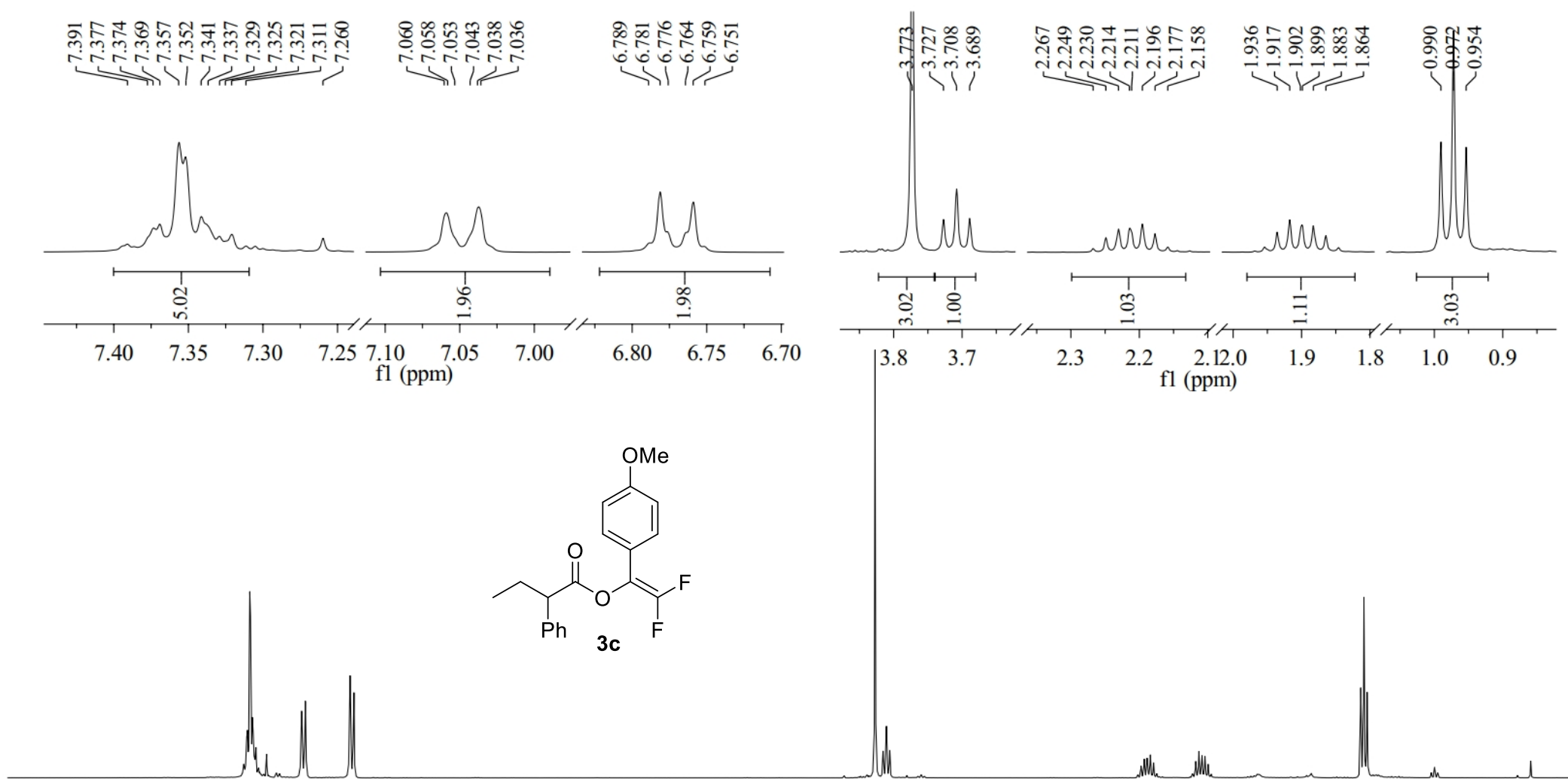

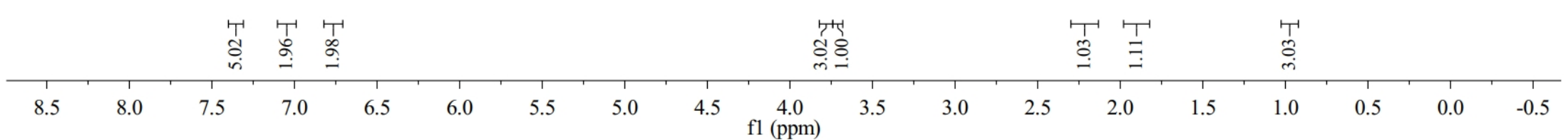

${ }^{1} \mathrm{H}$ NMR of Compound $\mathbf{3 c}\left(400 \mathrm{MHz}, \mathrm{CDCl}_{3}\right)$ 

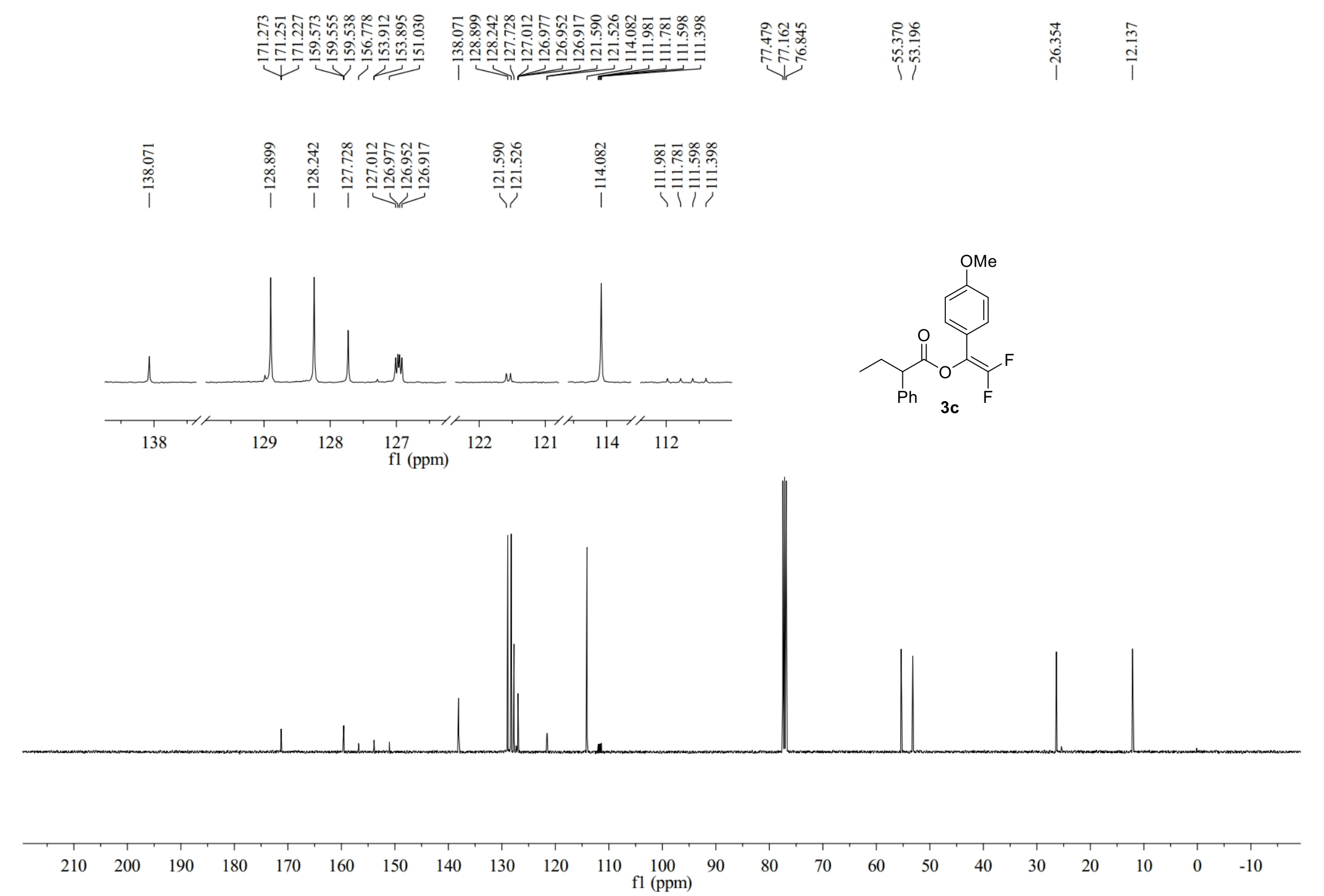

${ }^{13} \mathrm{C}$ NMR of Compound $3 \mathrm{c}\left(100 \mathrm{MHz}, \mathrm{CDCl}_{3}\right)$ 

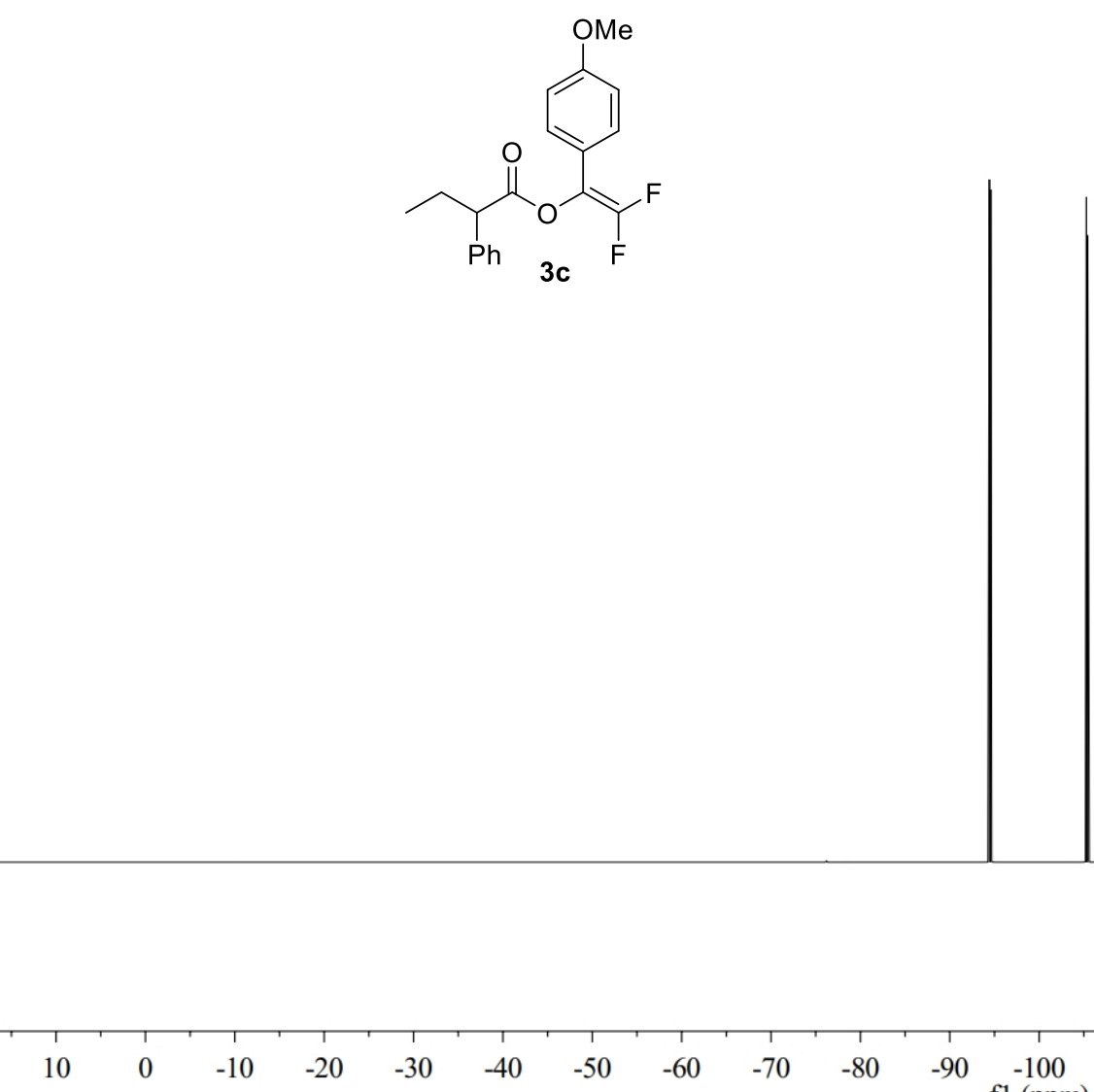

$\begin{array}{lll}-10 & -20 & -30\end{array}$

$-40$

$-50-60$

$-70 \quad-80$

$-90-100$

\begin{tabular}{llllllllllll}
\hline & -110 & -120 & -130 & -140 & -150 & -160 & -170 & -180 & -190 & -200 & -210
\end{tabular}

${ }^{19} \mathrm{~F}$ NMR of Compound $\mathbf{3 c}\left(376 \mathrm{MHz}, \mathrm{CDCl}_{3}\right)$ 


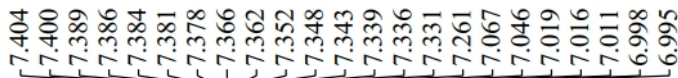
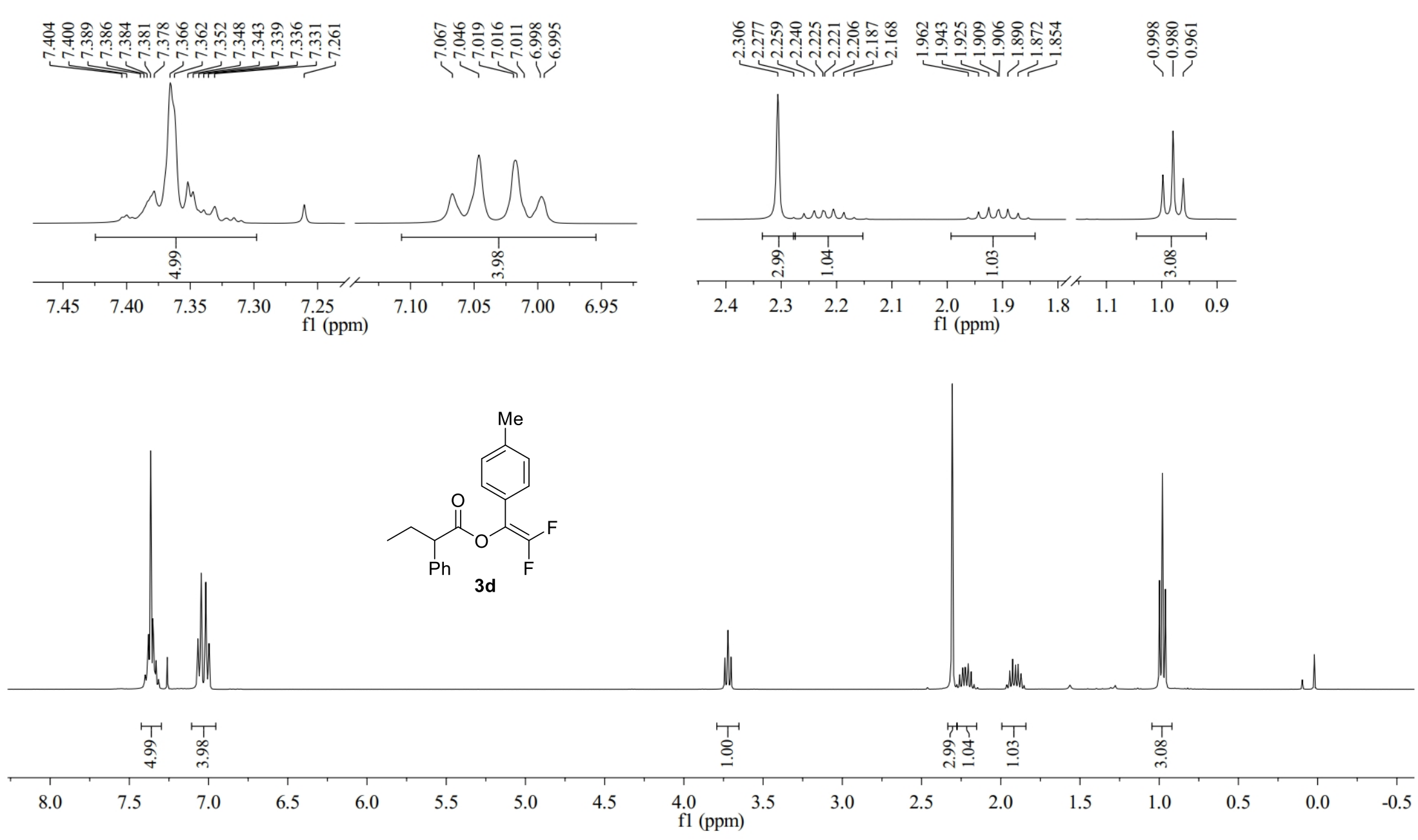

${ }^{1} \mathrm{H}$ NMR of Compound 3d (400 MHz, $\mathrm{CDCl}_{3}$ ) 

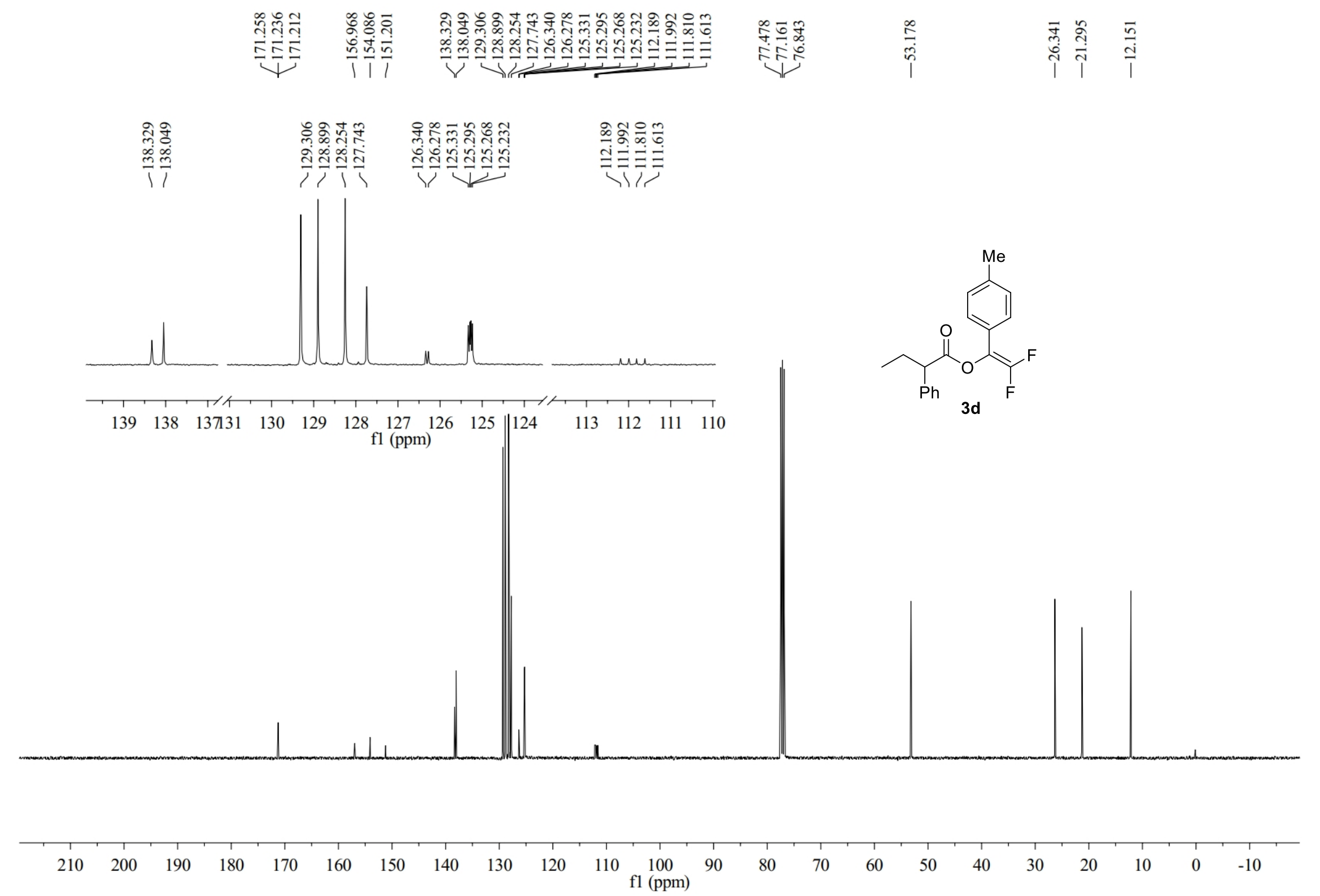

${ }^{13} \mathrm{C}$ NMR of Compound $\mathbf{3 d}\left(100 \mathrm{MHz}, \mathrm{CDCl}_{3}\right)$ 


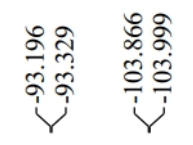

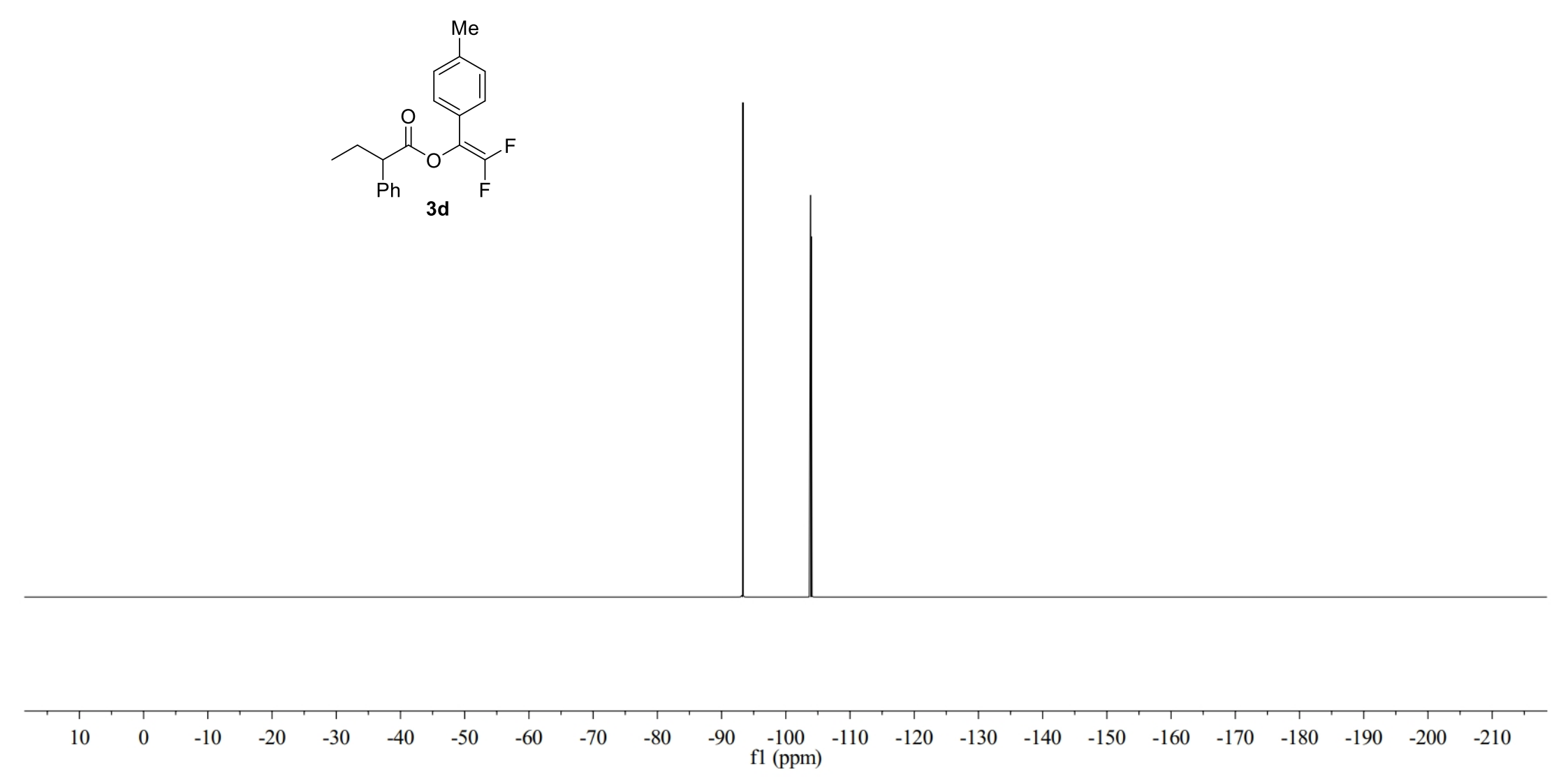

${ }^{19} \mathrm{~F}$ NMR of Compound 3d (376 MHz, $\left.\mathrm{CDCl}_{3}\right)$ 

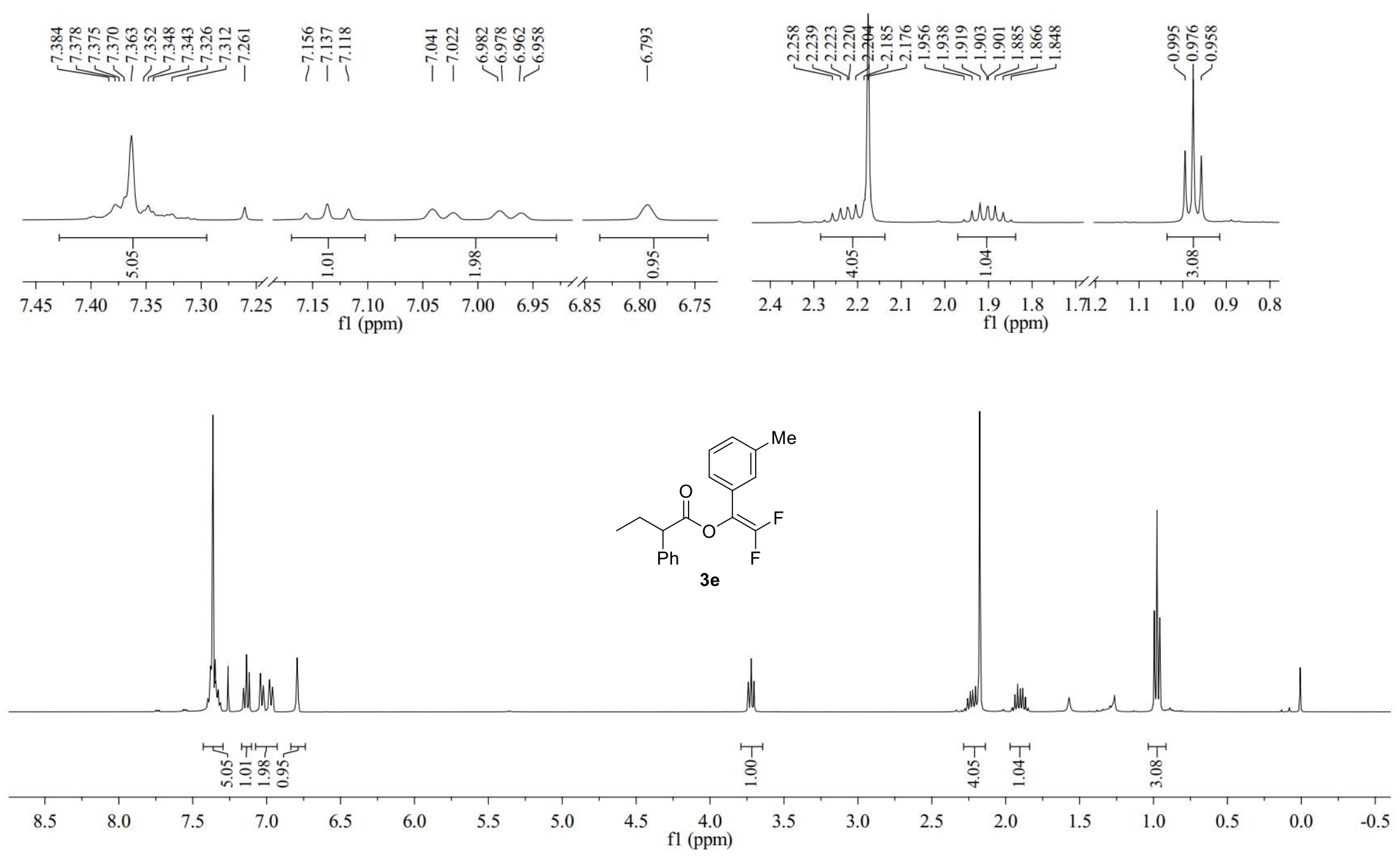

${ }^{1} \mathrm{H}$ NMR of Compound $3 \mathbf{e}\left(400 \mathrm{MHz}, \mathrm{CDCl}_{3}\right)$ 


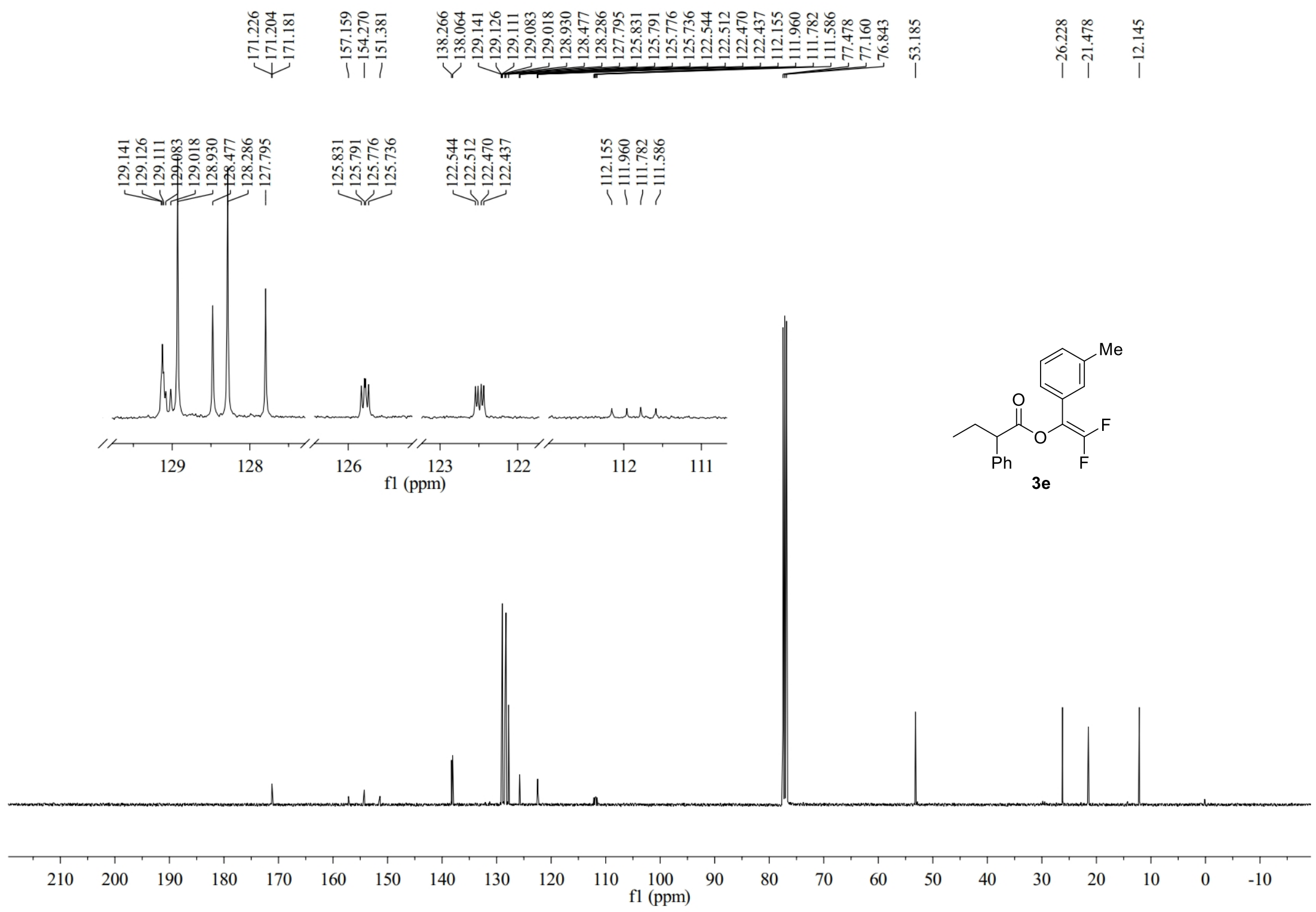

${ }^{13} \mathrm{C}$ NMR of Compound $3 e\left(100 \mathrm{MHz}, \mathrm{CDCl}_{3}\right)$ 


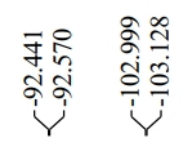

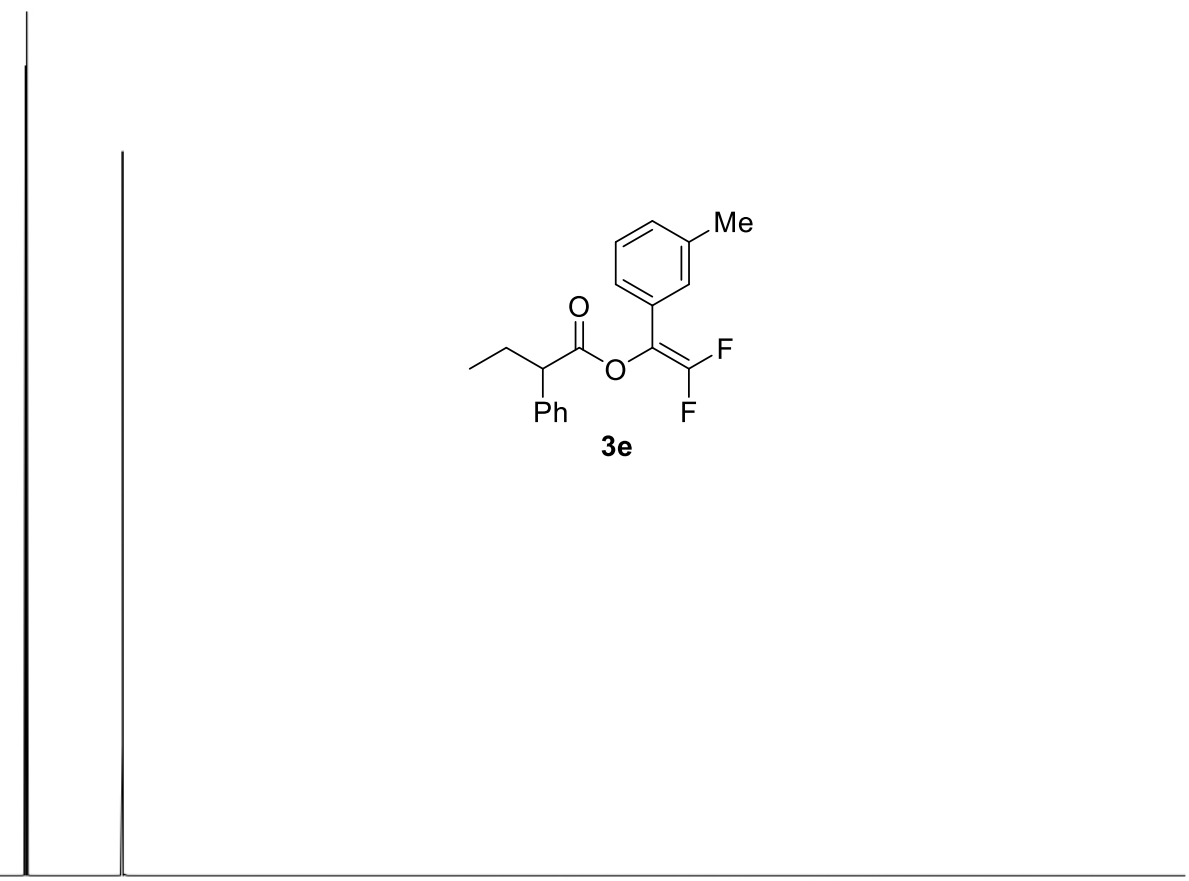

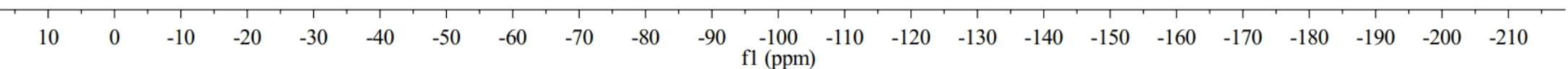

${ }^{19} \mathrm{~F}$ NMR of Compound $\mathbf{3 e}\left(376 \mathrm{MHz}, \mathrm{CDCl}_{3}\right)$ 


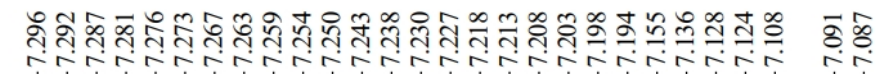

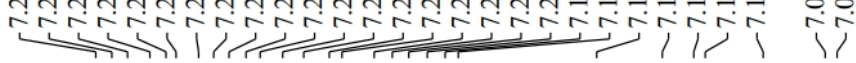

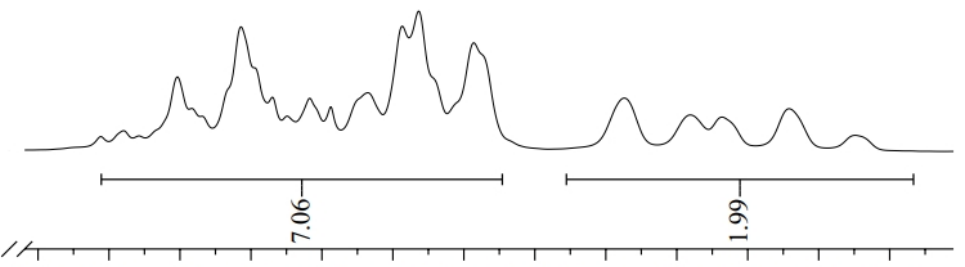

$\begin{array}{lllllllllllllllll}7.32 & 7.30 & 7.28 & 7.26 & 7.24 & 7.22 & 7.20 & 7.18 & 7.16 & 7.14 & 7.12 & 7.10 & 7.08\end{array}$ f1 (ppm)
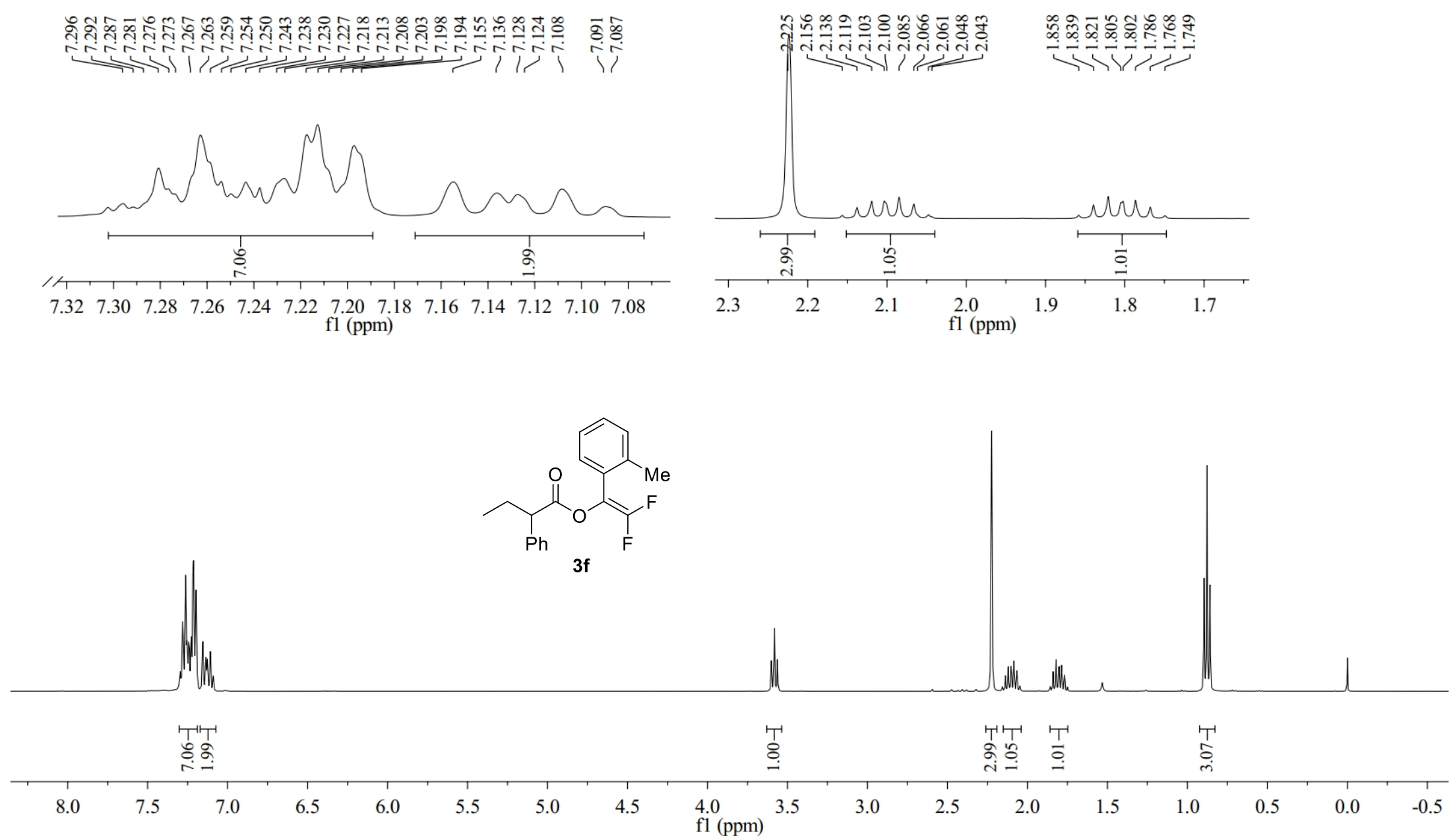

${ }^{1} \mathrm{H}$ NMR of Compound $\mathbf{3 f}\left(400 \mathrm{MHz}, \mathrm{CDCl}_{3}\right)$ 

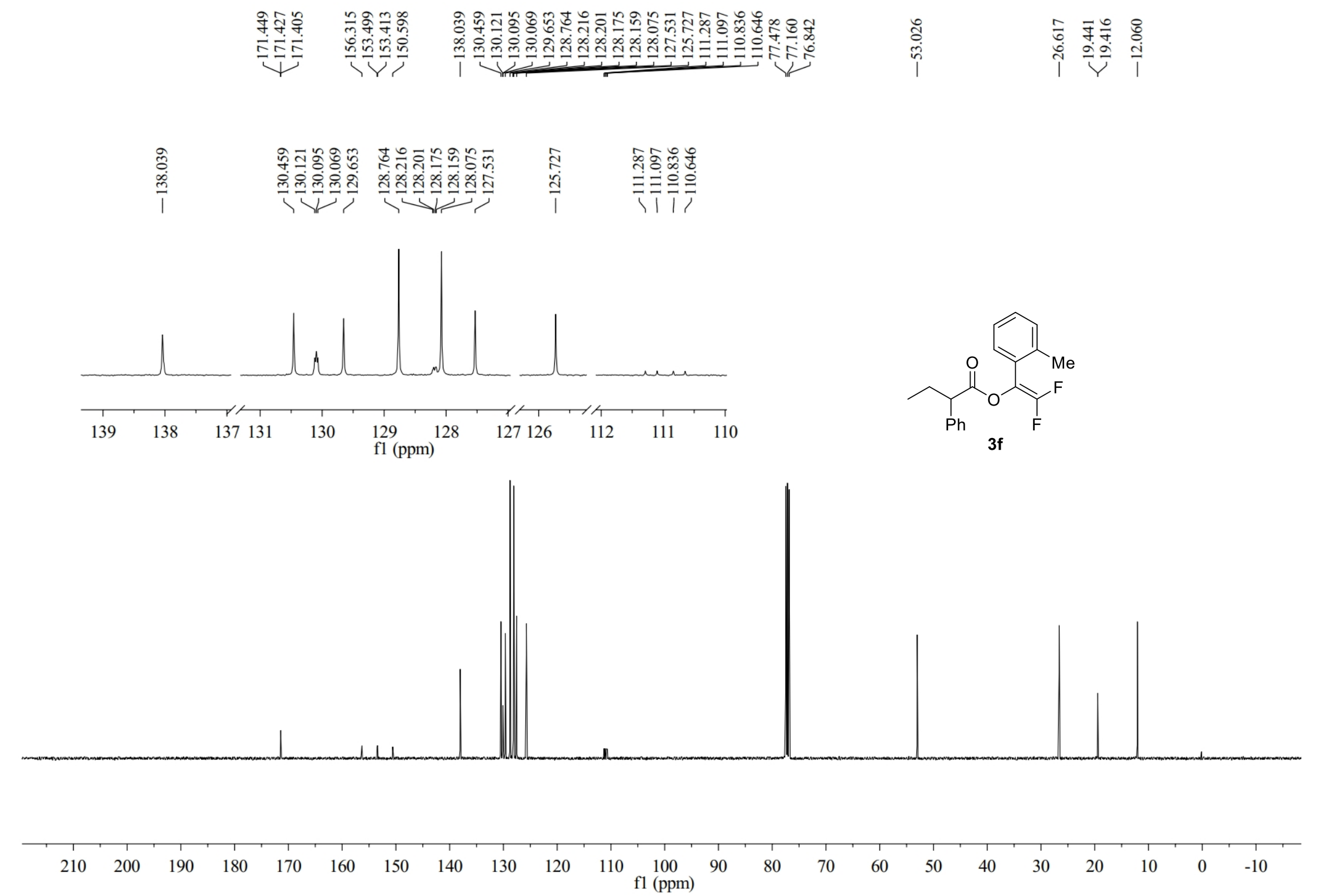

${ }^{13} \mathrm{C}$ NMR of Compound $\mathbf{3 f}\left(100 \mathrm{MHz}, \mathrm{CDCl}_{3}\right)$ 


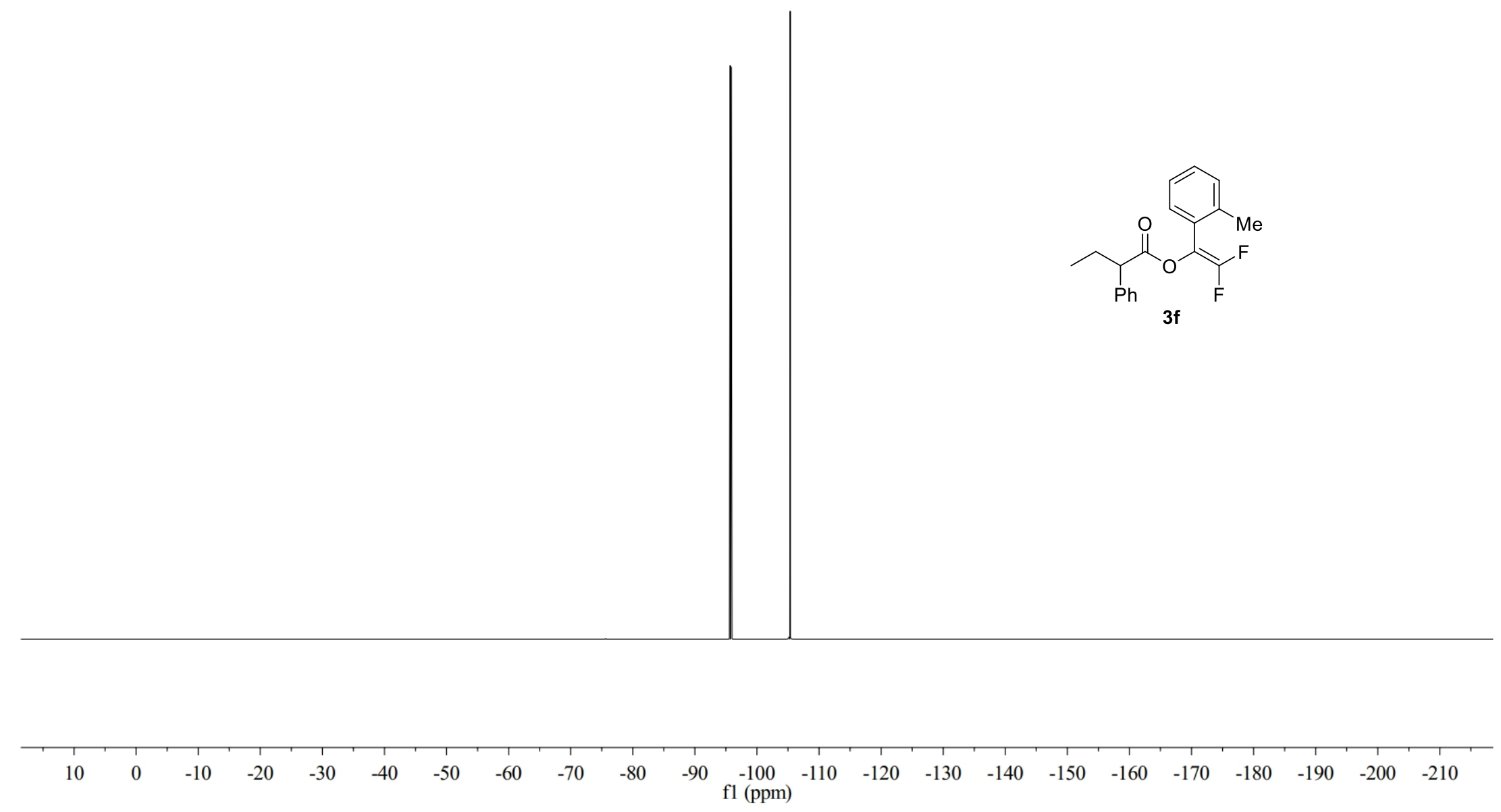

${ }^{19} \mathrm{~F}$ NMR of Compound $\mathbf{3 f}\left(376 \mathrm{MHz}, \mathrm{CDCl}_{3}\right)$ 


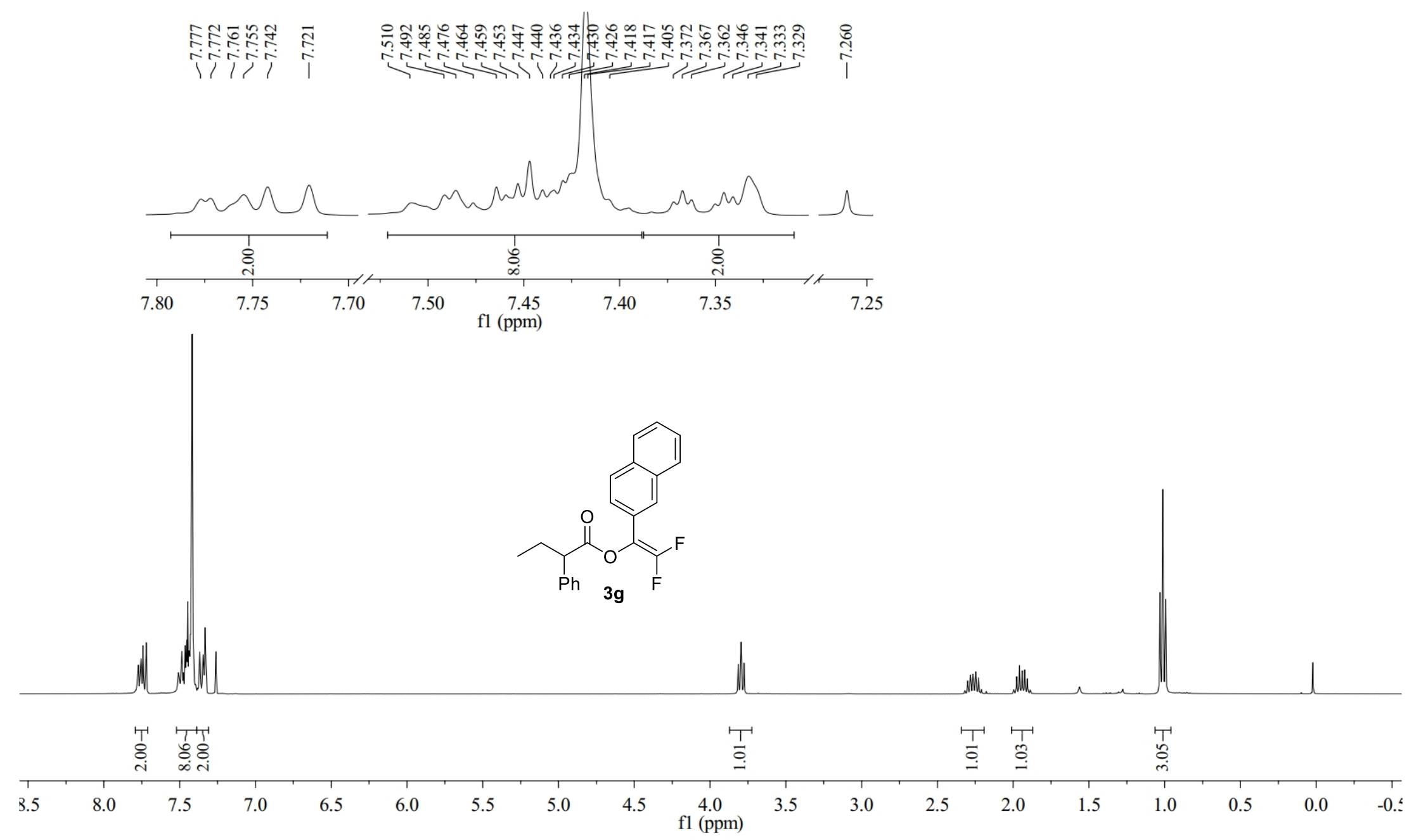

${ }^{1} \mathrm{H}$ NMR of Compound $\mathbf{3 g}\left(400 \mathrm{MHz}, \mathrm{CDCl}_{3}\right)$ 


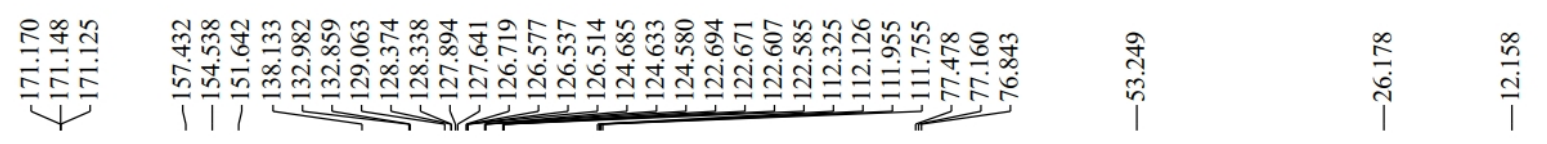

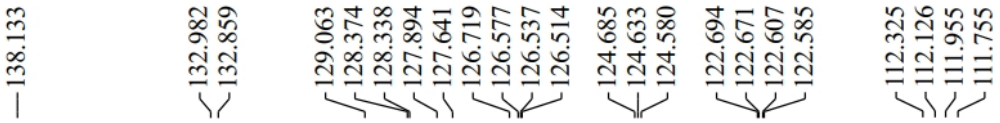

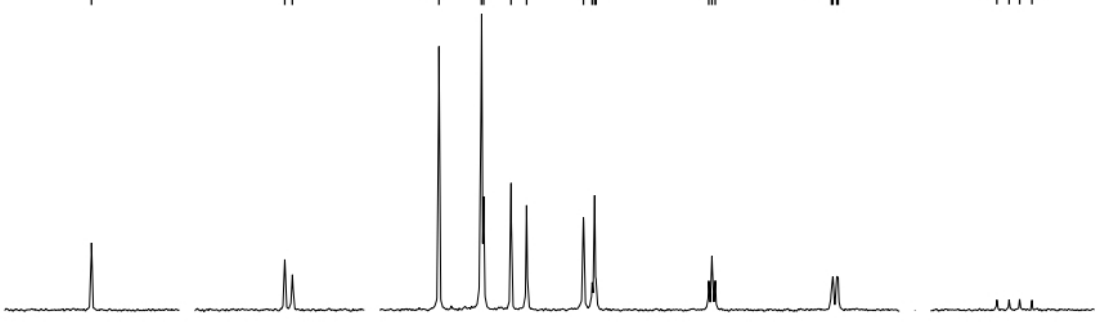

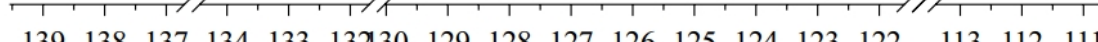

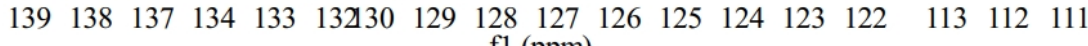
f1 (ppm)
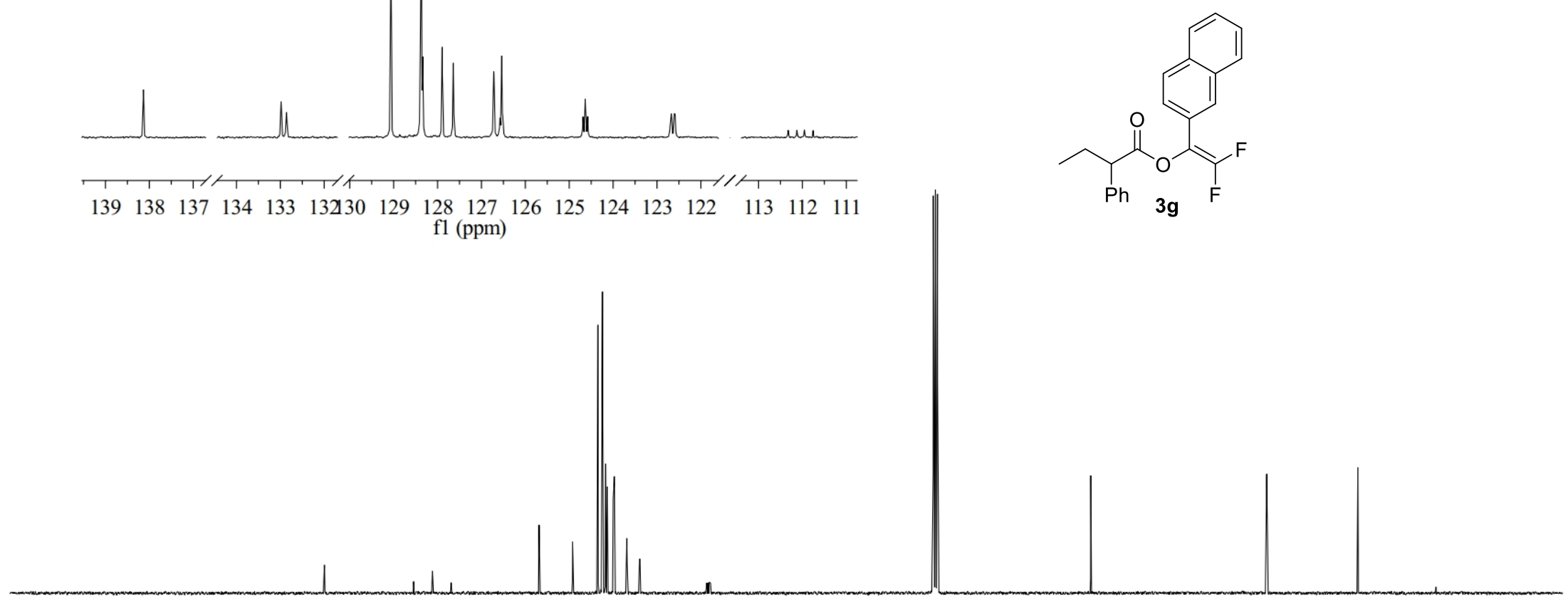

210

140

130

120

$110 \begin{aligned} & 100 \\ & \mathrm{fl}(\mathrm{ppm})\end{aligned}$

80

$70 \quad 60$

50

40

$30 \quad 20$

10

${ }^{13} \mathrm{C}$ NMR of Compound 3g (100 MHz, $\left.\mathrm{CDCl}_{3}\right)$ 


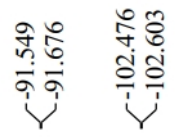

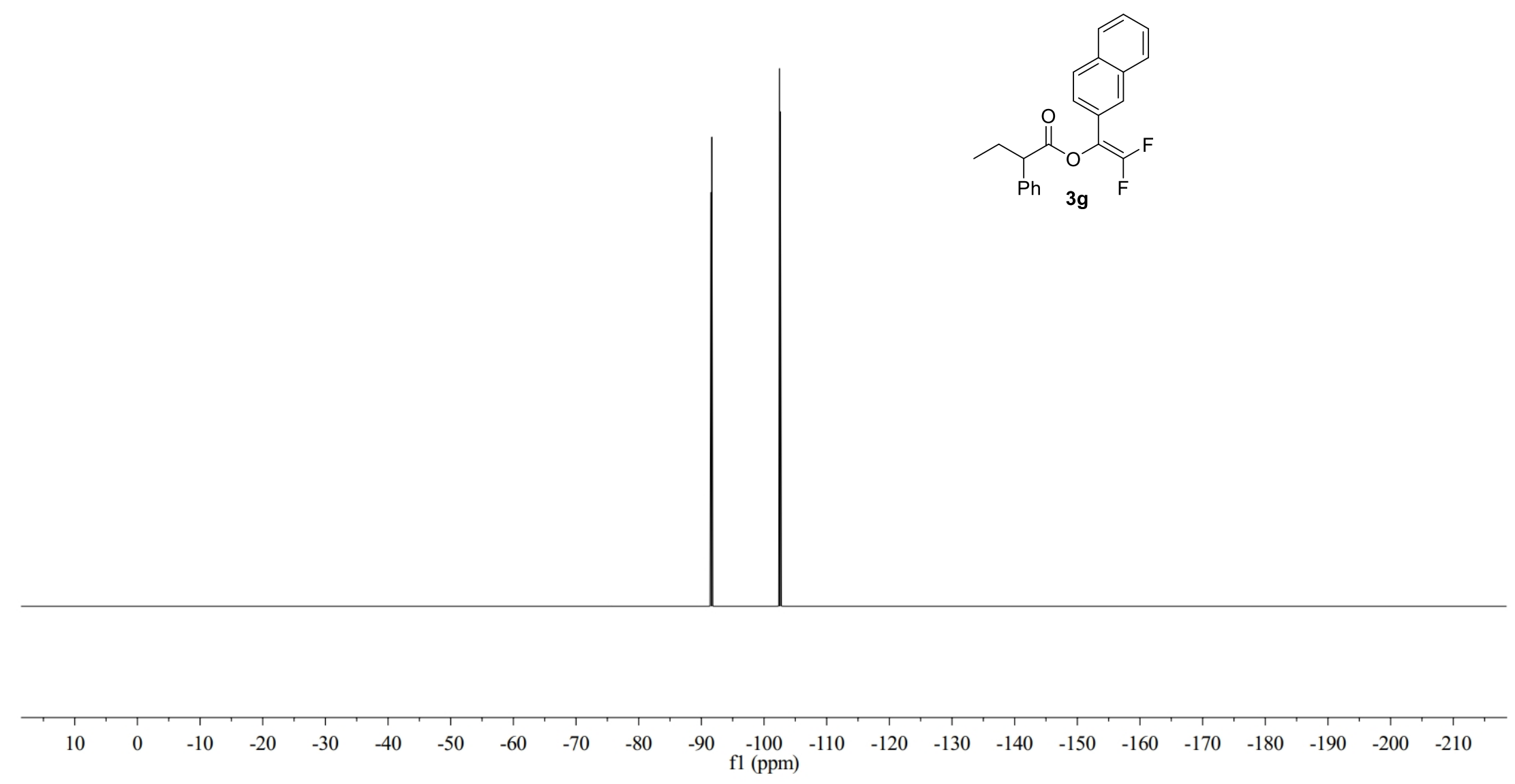

${ }^{19} \mathrm{~F}$ NMR of Compound $3 \mathrm{~g}$ (376 MHz, $\mathrm{CDCl}_{3}$ ) 


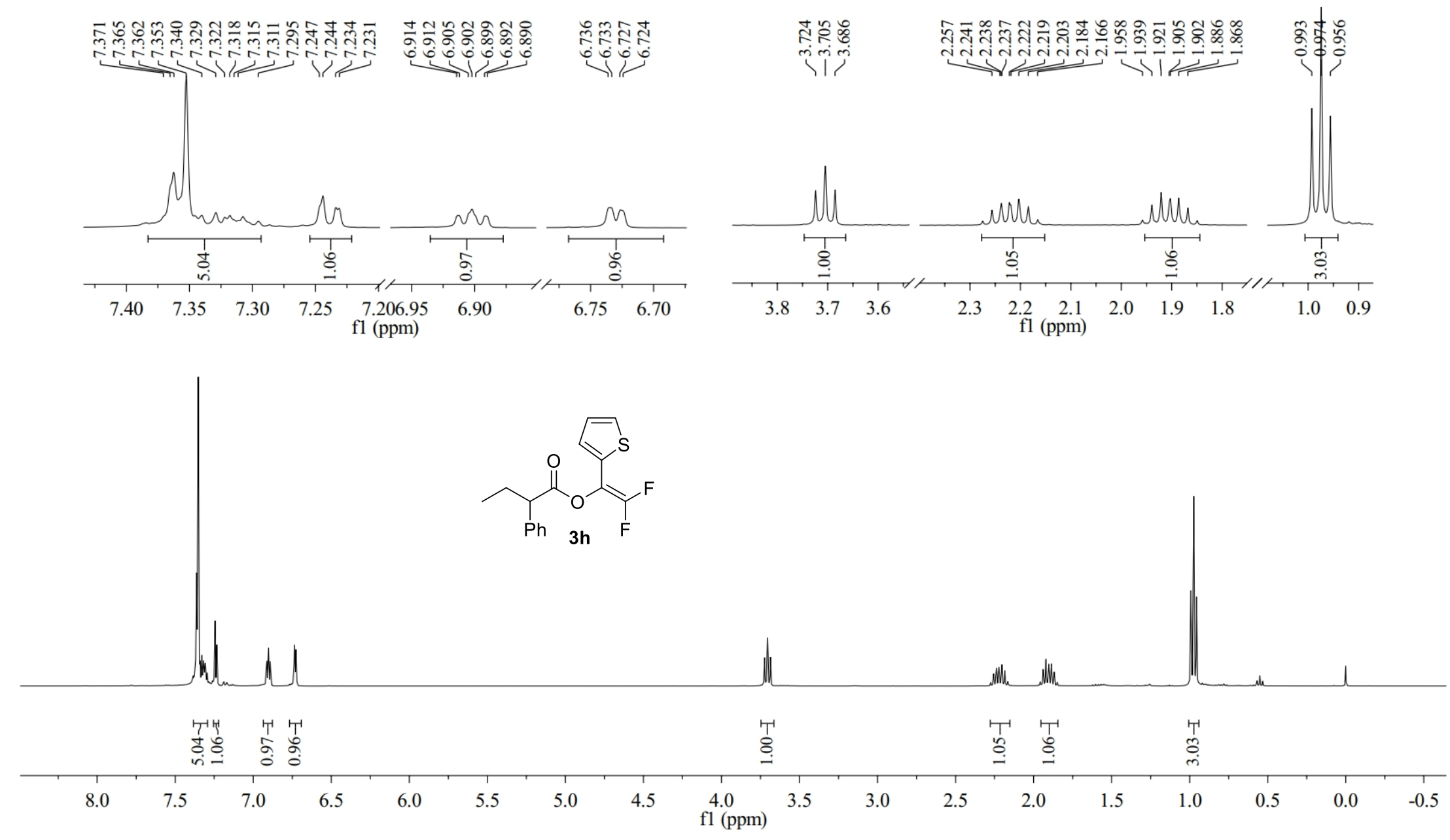

${ }^{1} \mathrm{H}$ NMR of Compound $\mathbf{3 h}\left(400 \mathrm{MHz}, \mathrm{CDCl}_{3}\right)$ 

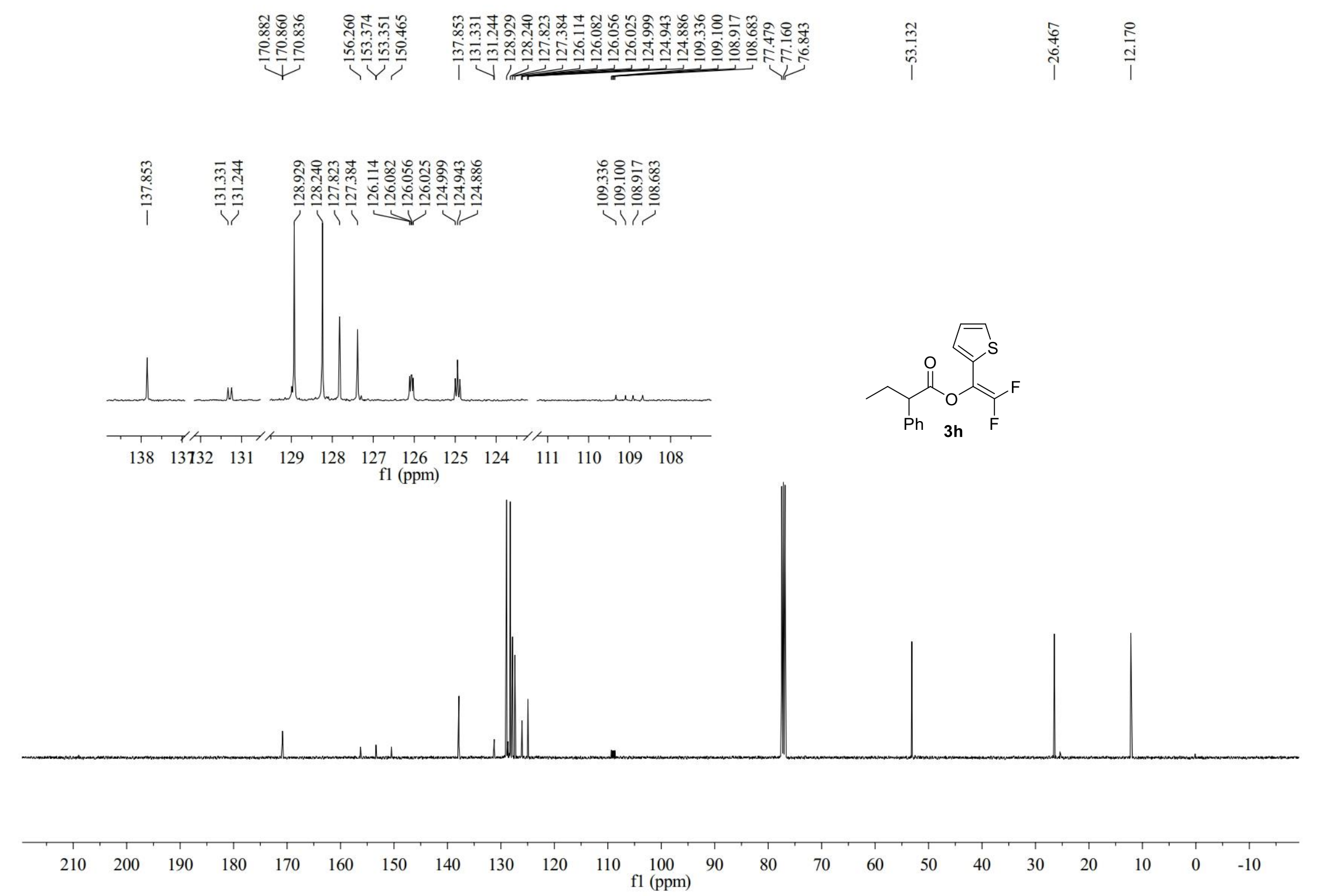

${ }^{13} \mathrm{C}$ NMR of Compound $\mathbf{3 h}\left(100 \mathrm{MHz}, \mathrm{CDCl}_{3}\right)$ 


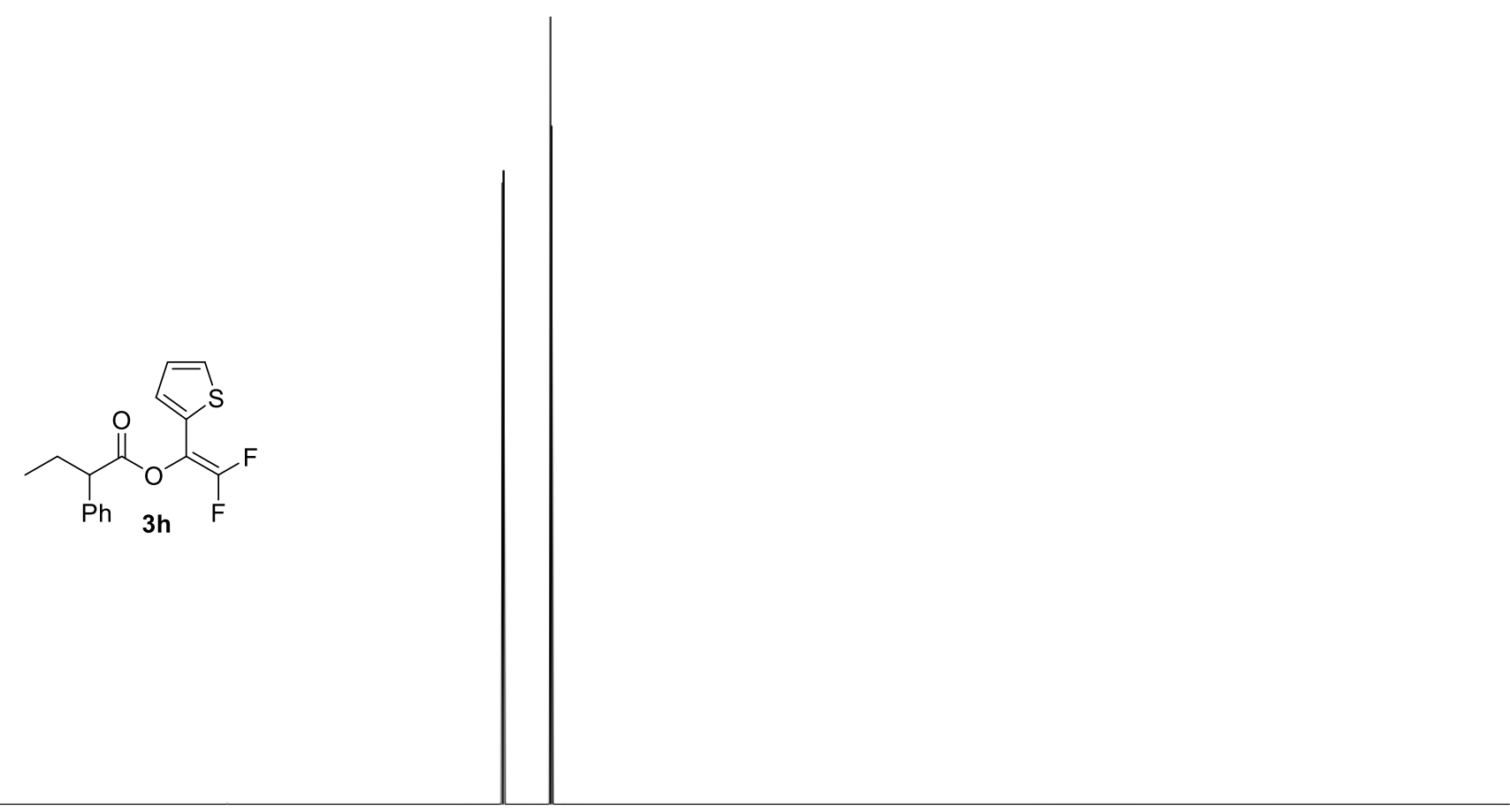

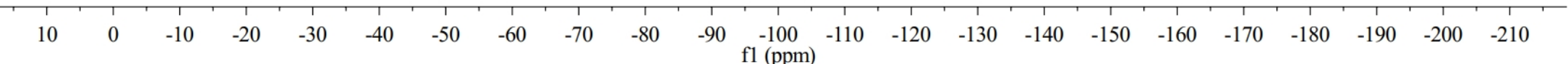

${ }^{19} \mathrm{~F}$ NMR of Compound $\mathbf{3 h}\left(376 \mathrm{MHz}, \mathrm{CDCl}_{3}\right)$ 

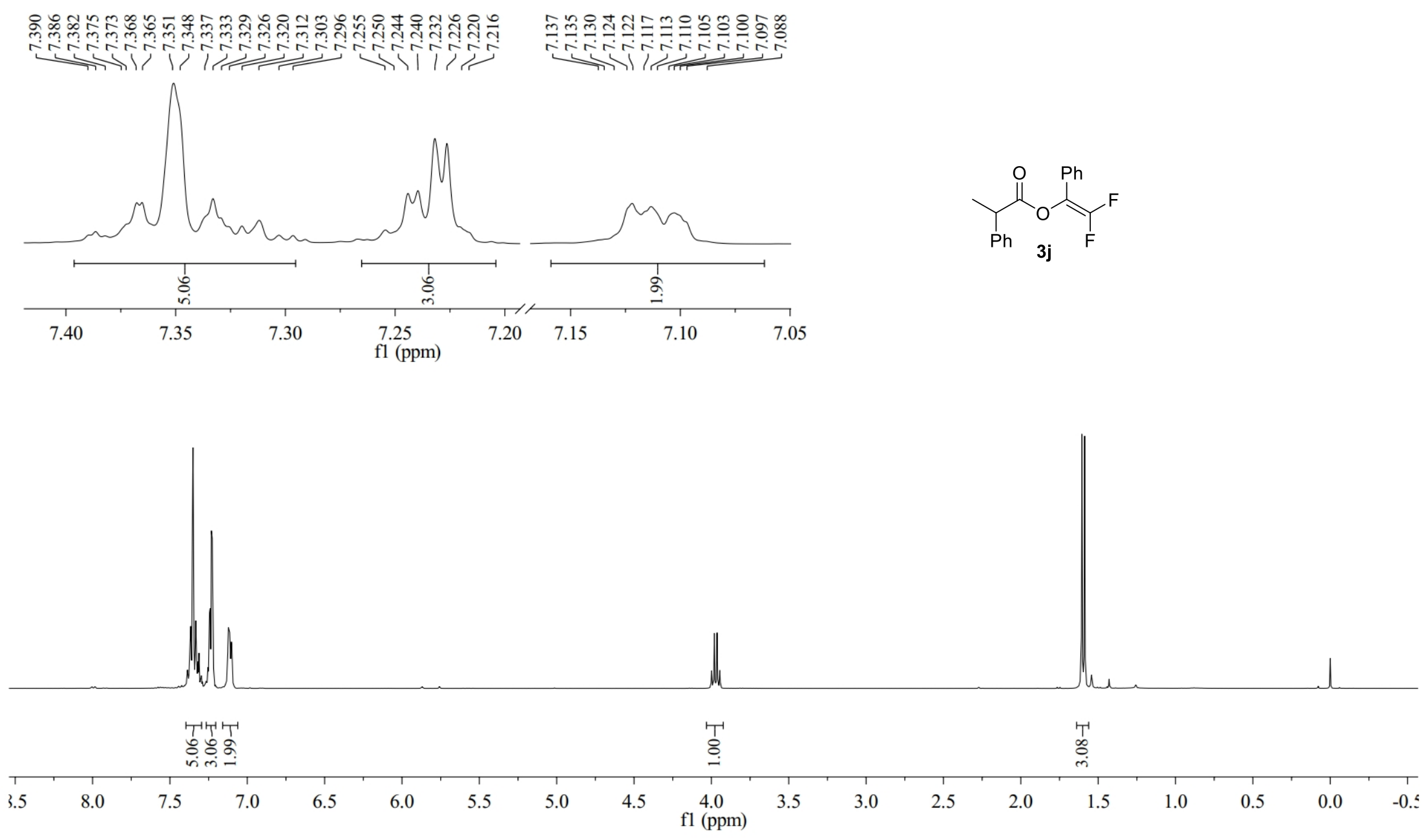

${ }^{1} \mathrm{H}$ NMR of Compound $\mathbf{3 j}$ (400 MHz, $\mathrm{CDCl}_{3}$ ) 

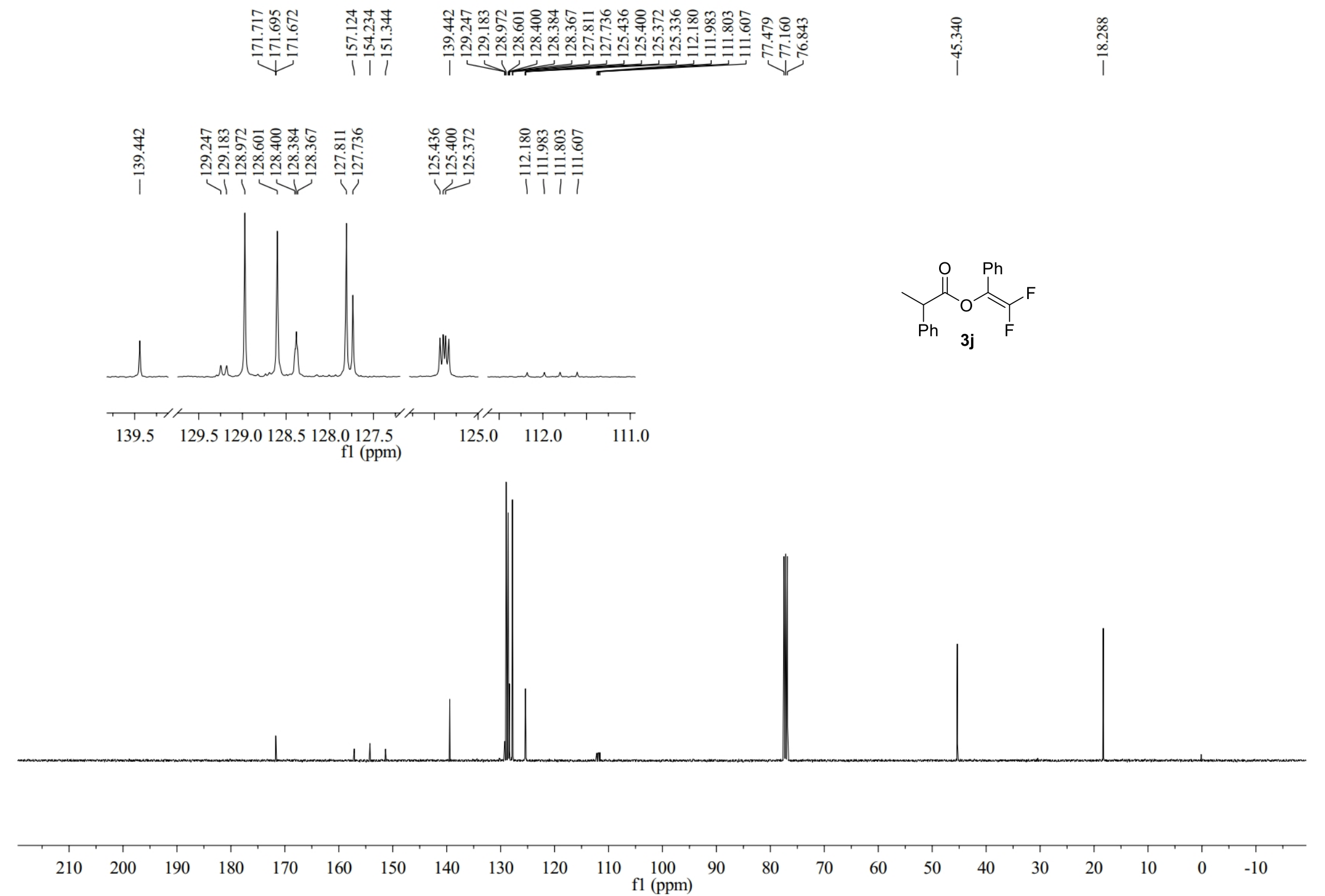

${ }^{13} \mathrm{C}$ NMR of Compound $\mathbf{3 j}$ (100 MHz, $\mathrm{CDCl}_{3}$ ) 


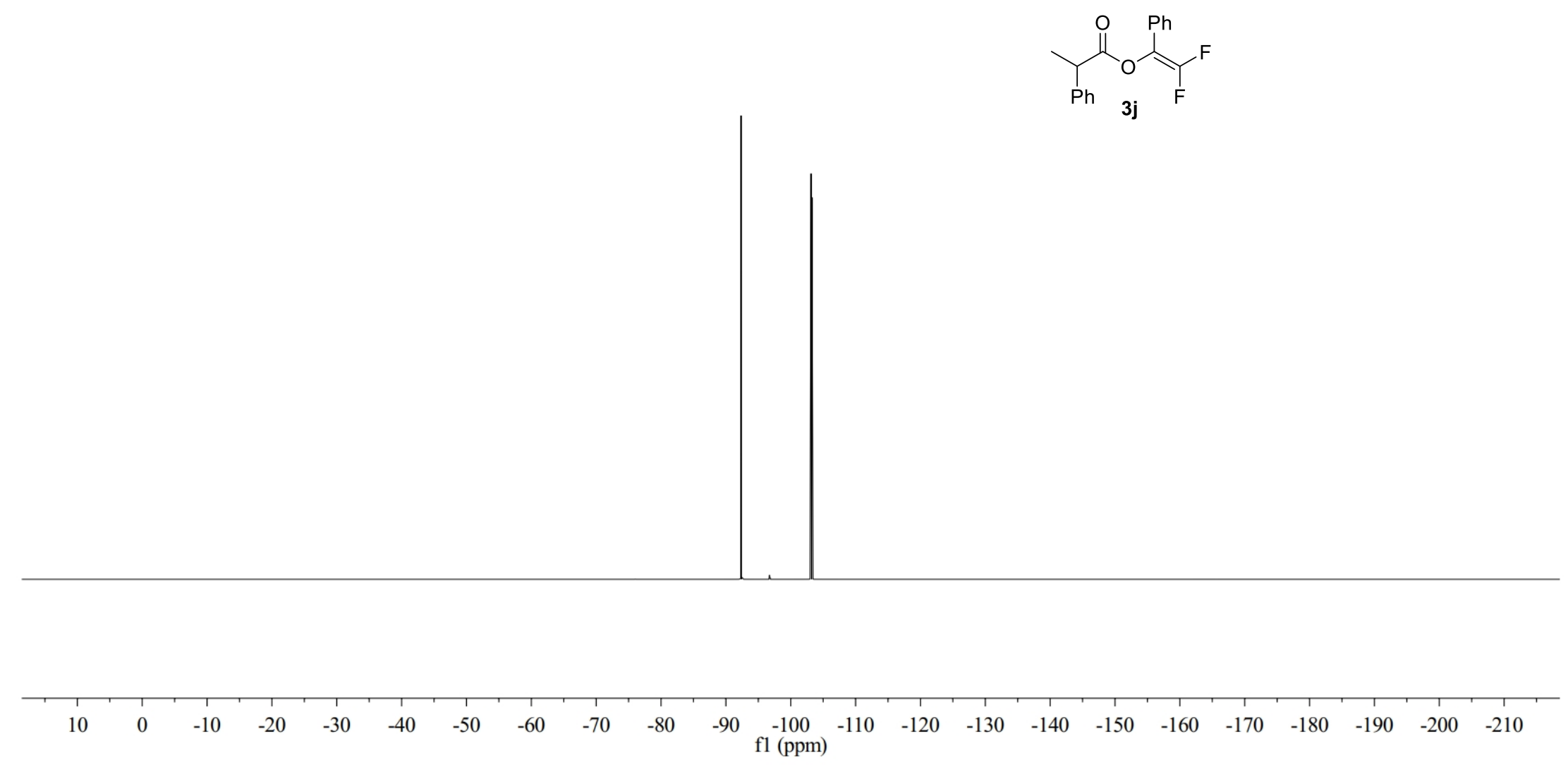

${ }^{19} \mathrm{~F}$ NMR of Compound $\mathbf{3 j}$ (376 MHz, $\left.\mathrm{CDCl}_{3}\right)$ 


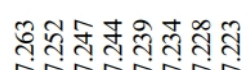

帝

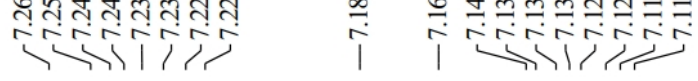
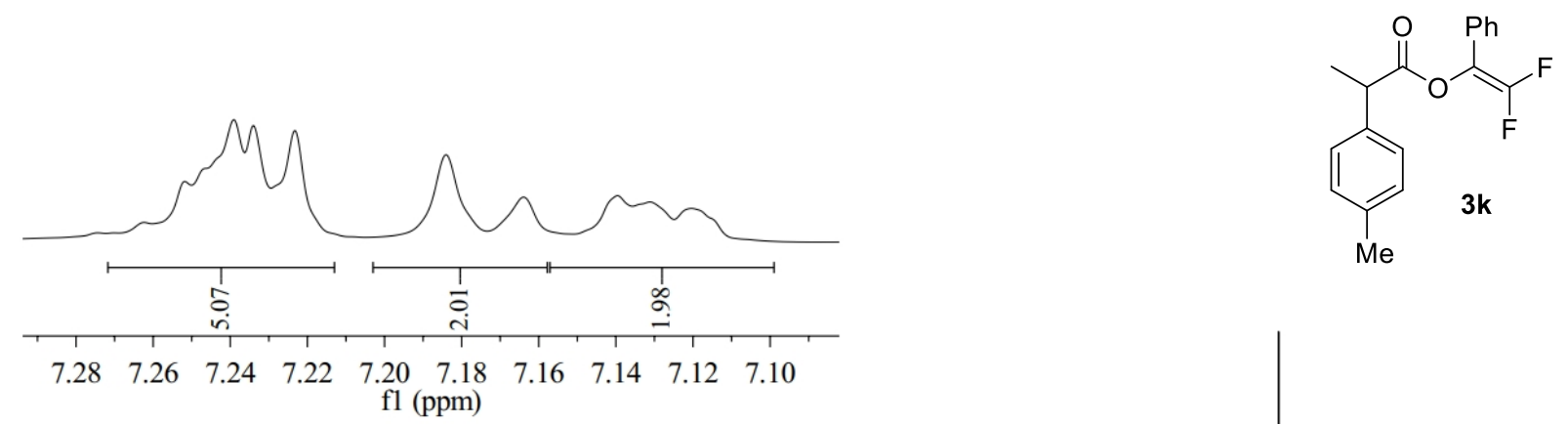

$\begin{array}{llllllllll}7.28 & 7.26 & 7.24 & 7.22 & \begin{array}{c}7.20 \\ \text { fl }\end{array} & \begin{array}{lllll}7.18 \\ (\mathrm{ppm})\end{array} & 7.16 & 7.14 & 7.12 & 7.10\end{array}$
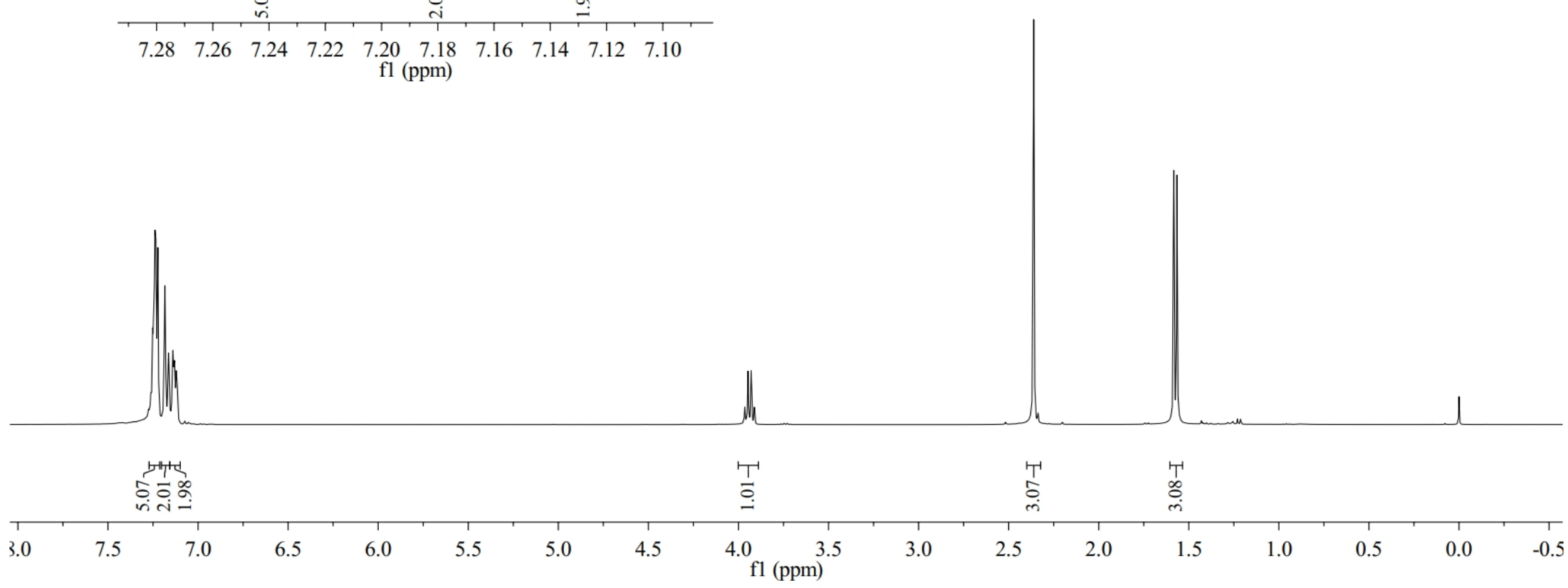

${ }^{1} \mathrm{H}$ NMR of Compound 3k (400 MHz, $\mathrm{CDCl}_{3}$ ) 


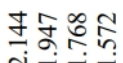

要主
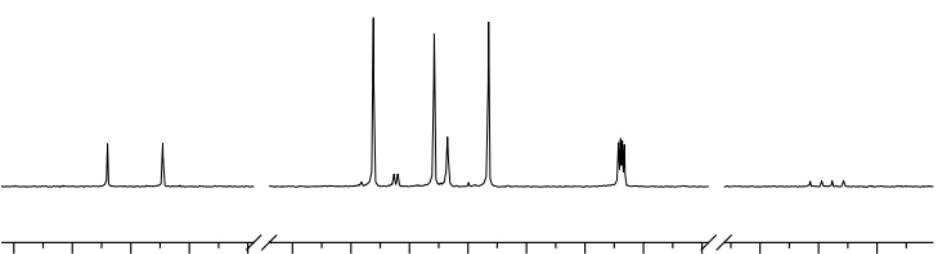

139

$\begin{array}{llllllllllll}137 & 135131 & 130 & 129 & 128 & 127 & 126 & 125 & 124 & 113 & 112 & 111\end{array}$ fl (ppm)
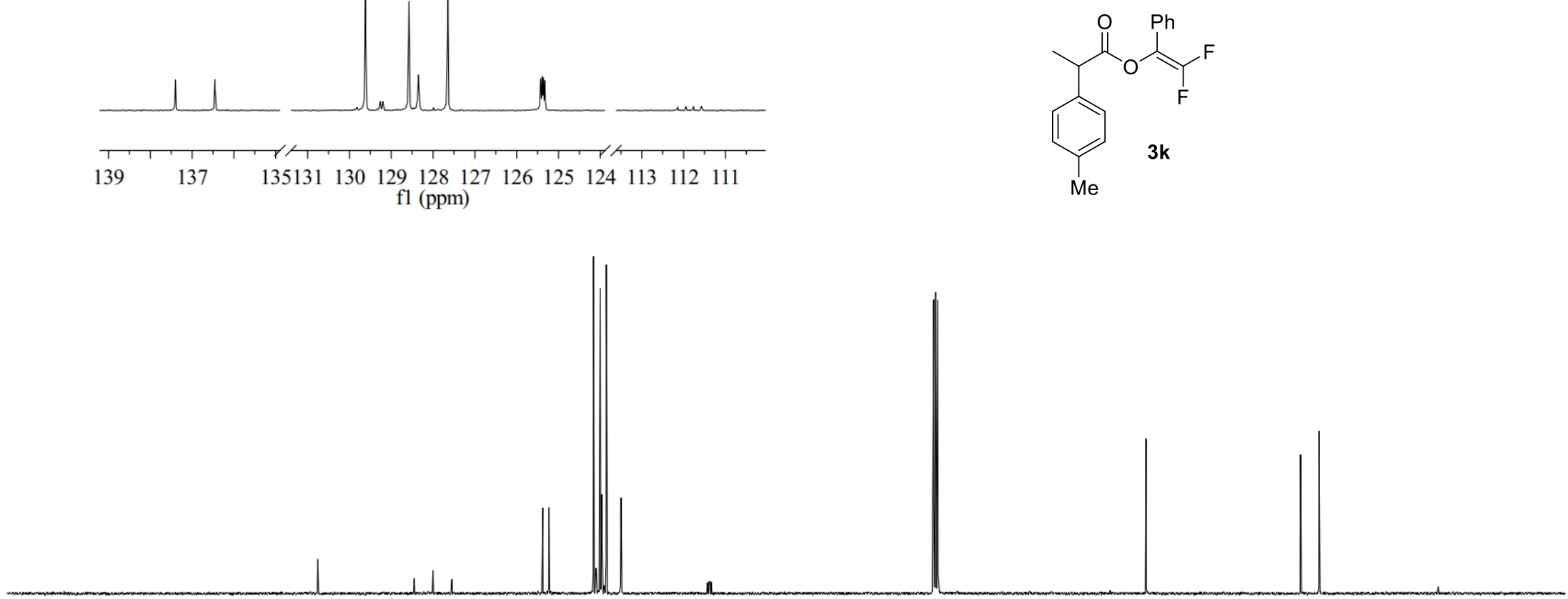

210

$200 \quad 190 \quad 180 \quad 170$

${ }^{13} \mathrm{C}$ NMR of Compound 3k (100 MHz, $\left.\mathrm{CDCl}_{3}\right)$ 


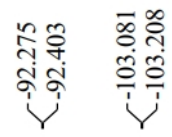
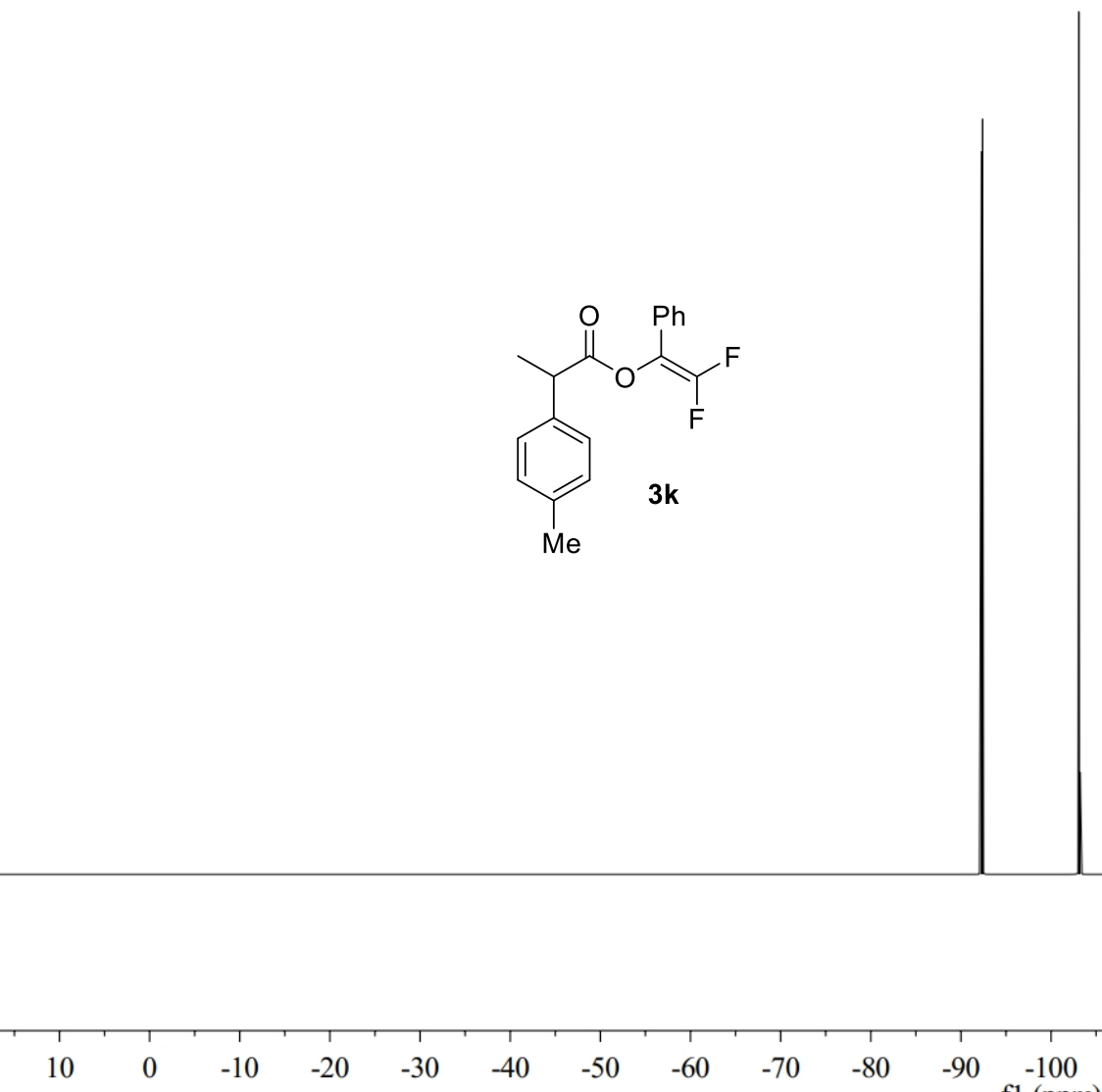

$\begin{array}{llll}-10 & -20 & -30 & -40\end{array}$

$-50$

$-60$

$-80$

$-90-100$

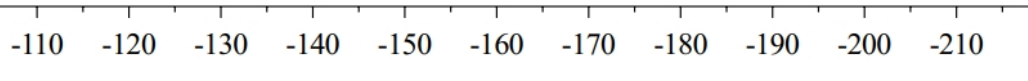

${ }^{19} \mathrm{~F}$ NMR of Compound 3k (376 MHz, $\mathrm{CDCl}_{3}$ ) 


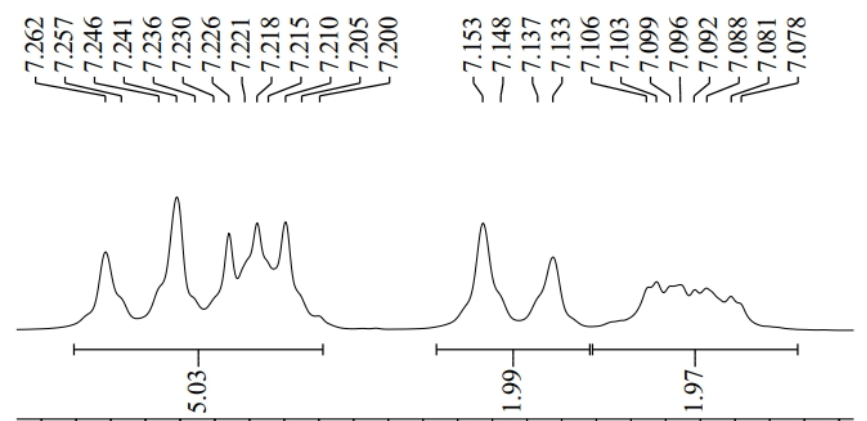

$\begin{array}{llllllllllllll}7.28 & 7.26 & 7.24 & 7.22 & 7.20 & 7.18 & 7.16 & 7.14 & 7.12 & 7.10 & 7.08 & 7.06\end{array}$
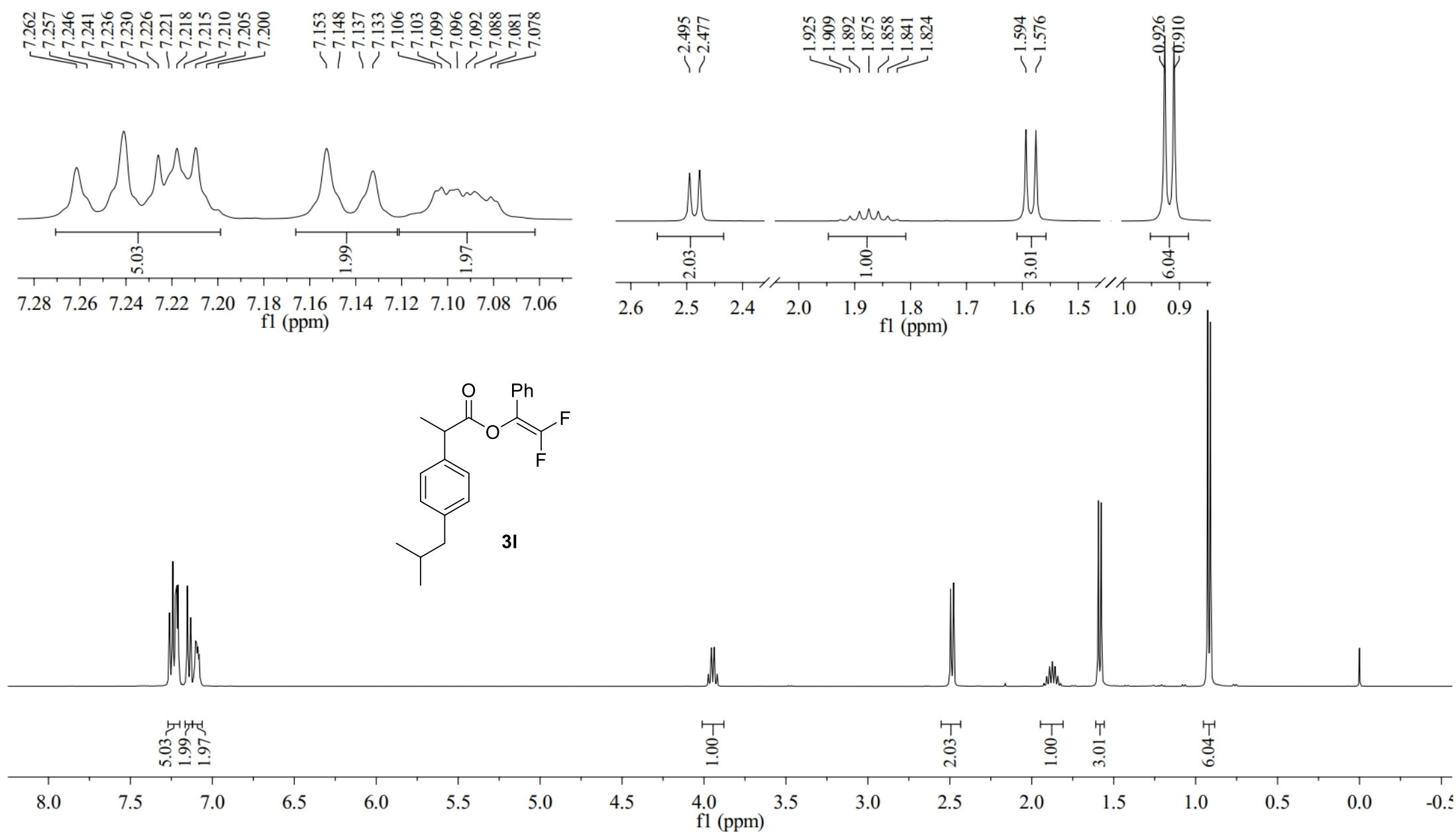

${ }^{1} \mathrm{H}$ NMR of Compound 31 (400 MHz, $\mathrm{CDCl}_{3}$ ) 

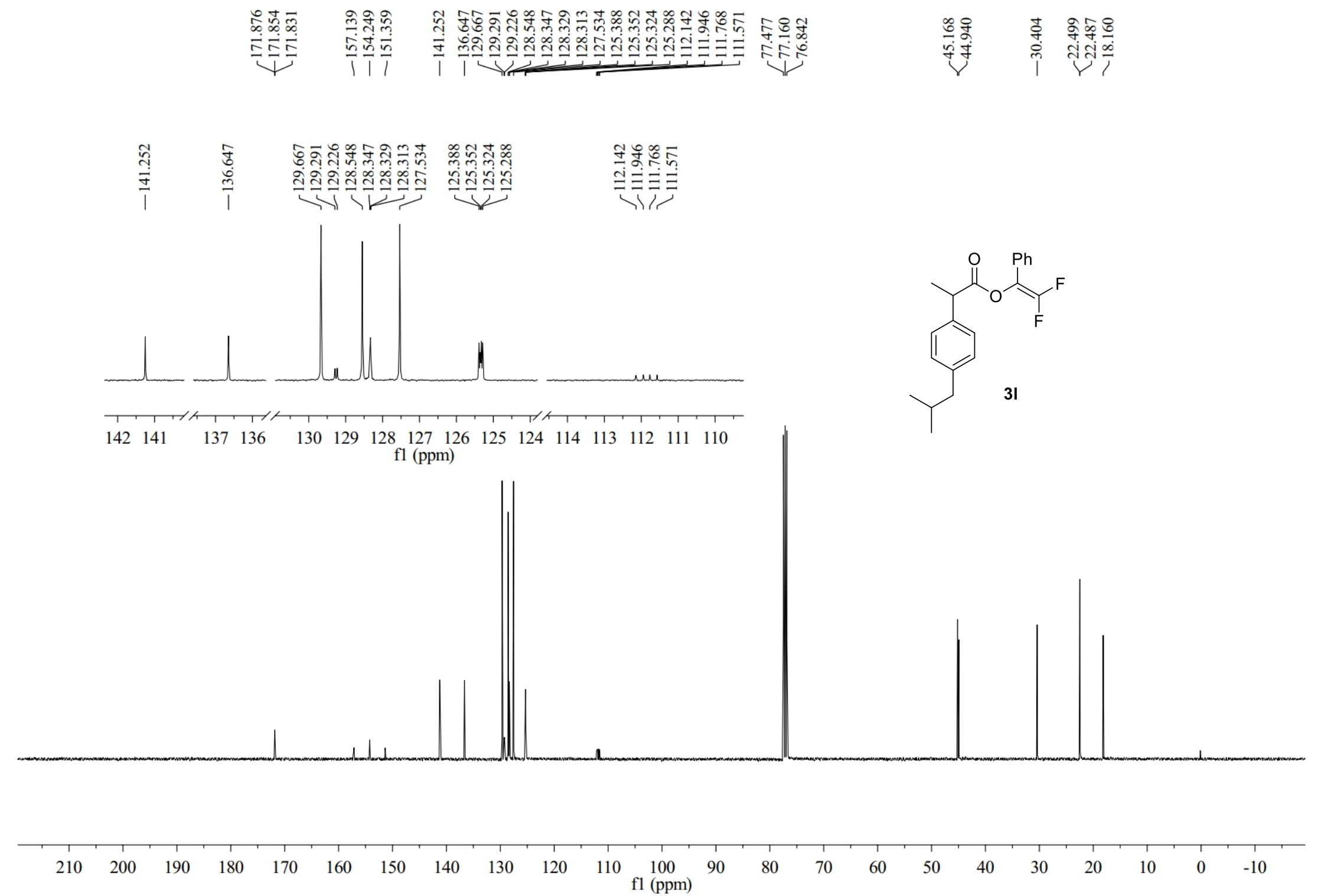

${ }^{13} \mathrm{C}$ NMR of Compound $3 \mathrm{l}\left(100 \mathrm{MHz}, \mathrm{CDCl}_{3}\right)$ 

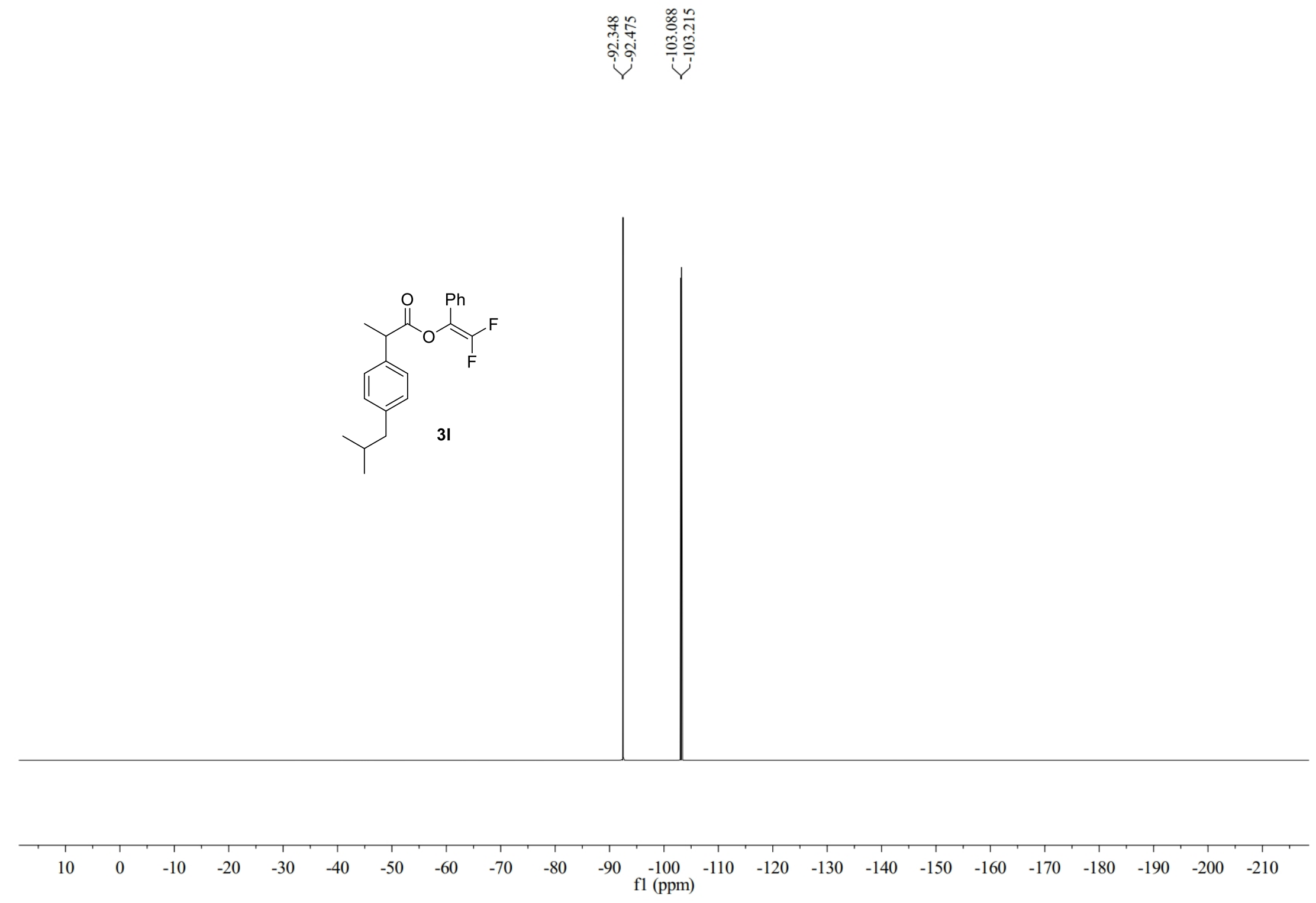

${ }^{19} \mathrm{~F}$ NMR of Compound 31 ( $\left.376 \mathrm{MHz}, \mathrm{CDCl}_{3}\right)$ 

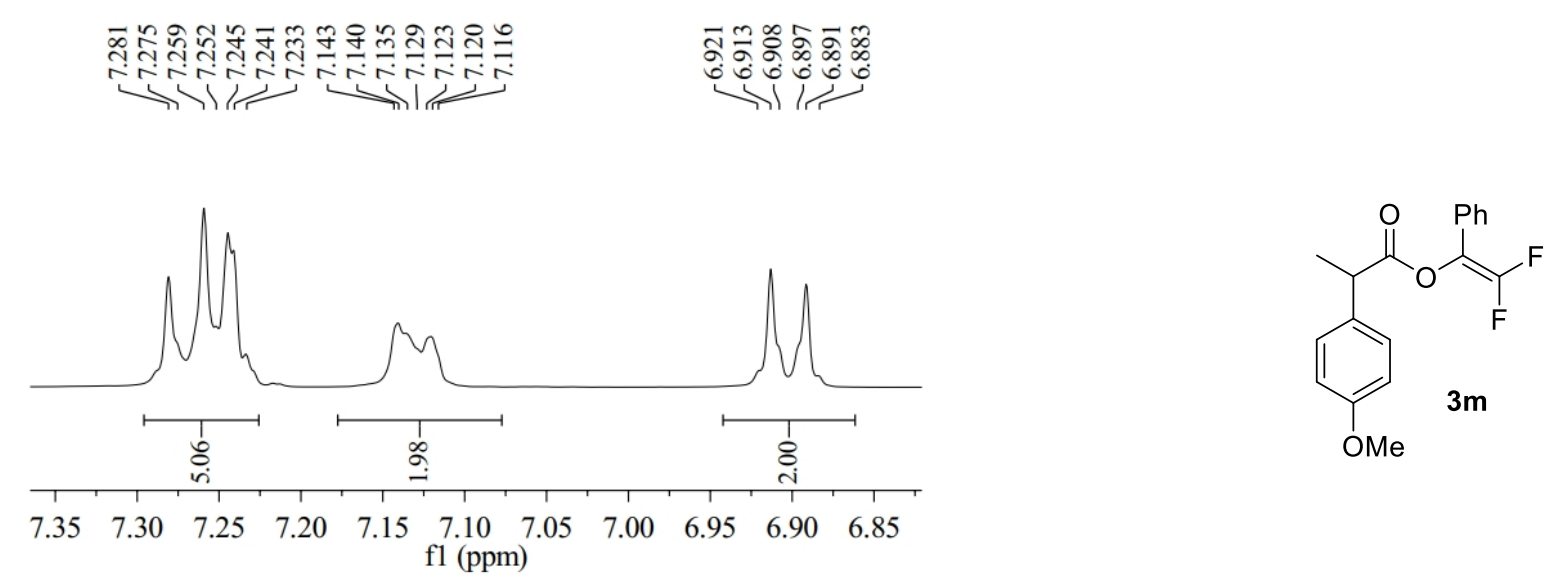

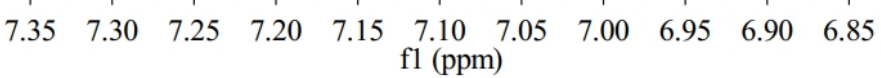

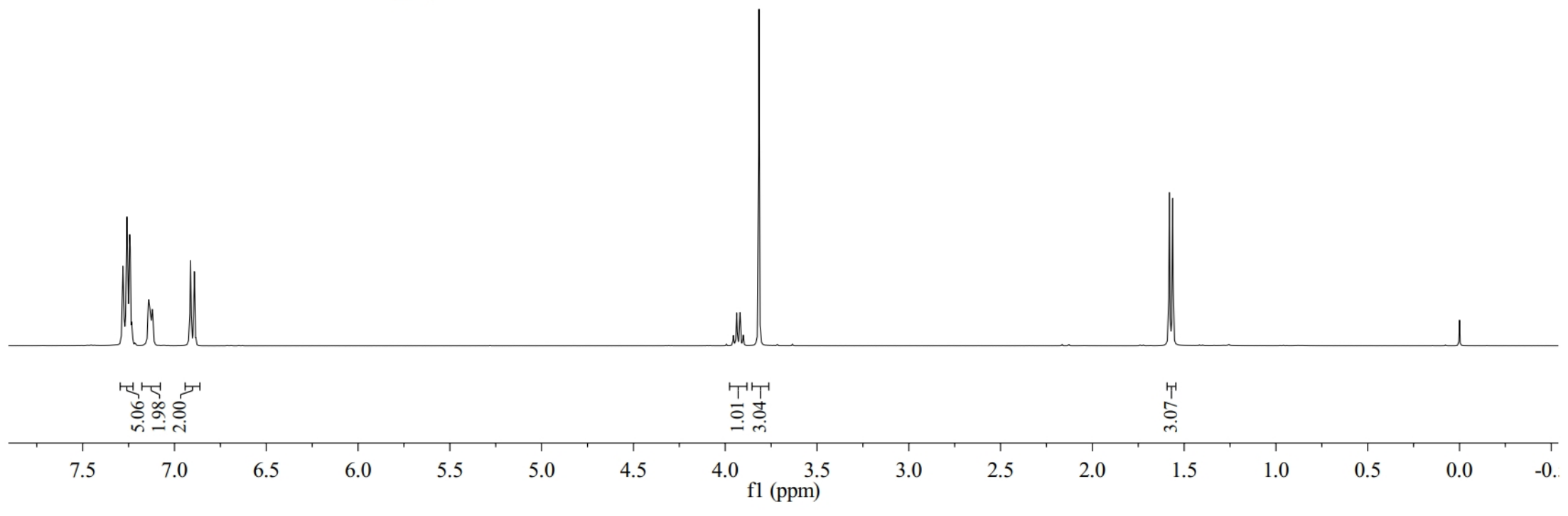

${ }^{1} \mathrm{H}$ NMR of Compound $\mathbf{3 m}\left(400 \mathrm{MHz}, \mathrm{CDCl}_{3}\right)$ 

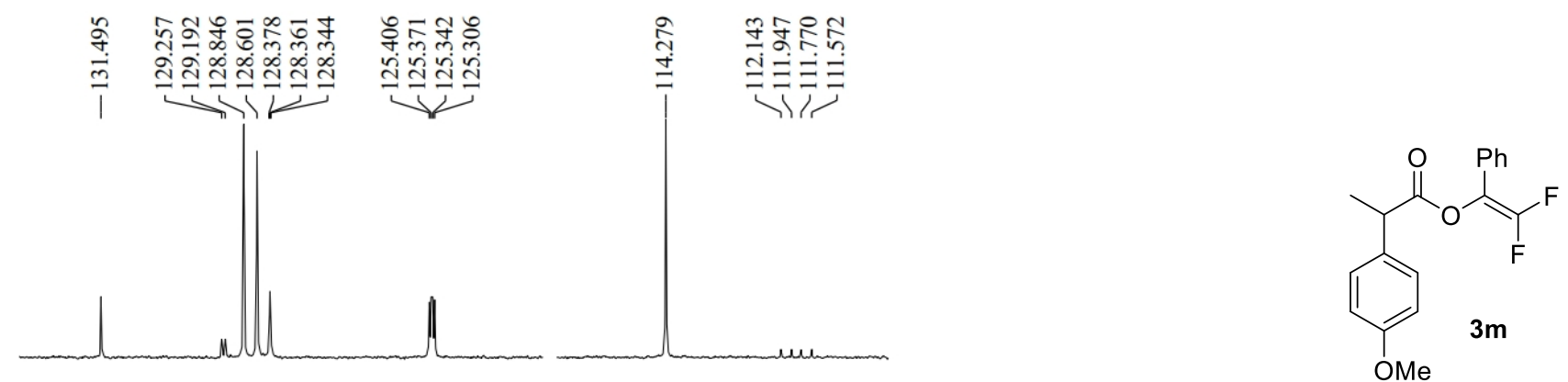
$\mathrm{fl}(\mathrm{ppm})$

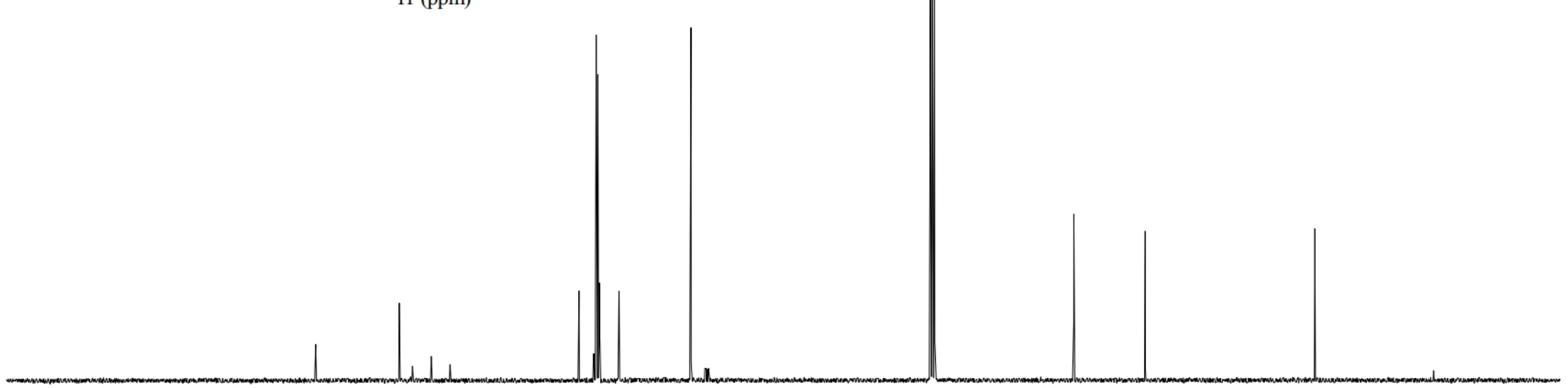

${ }^{13} \mathrm{C}$ NMR of Compound $3 \mathbf{m}\left(100 \mathrm{MHz}, \mathrm{CDCl}_{3}\right)$ 


$$
\text { نู }
$$

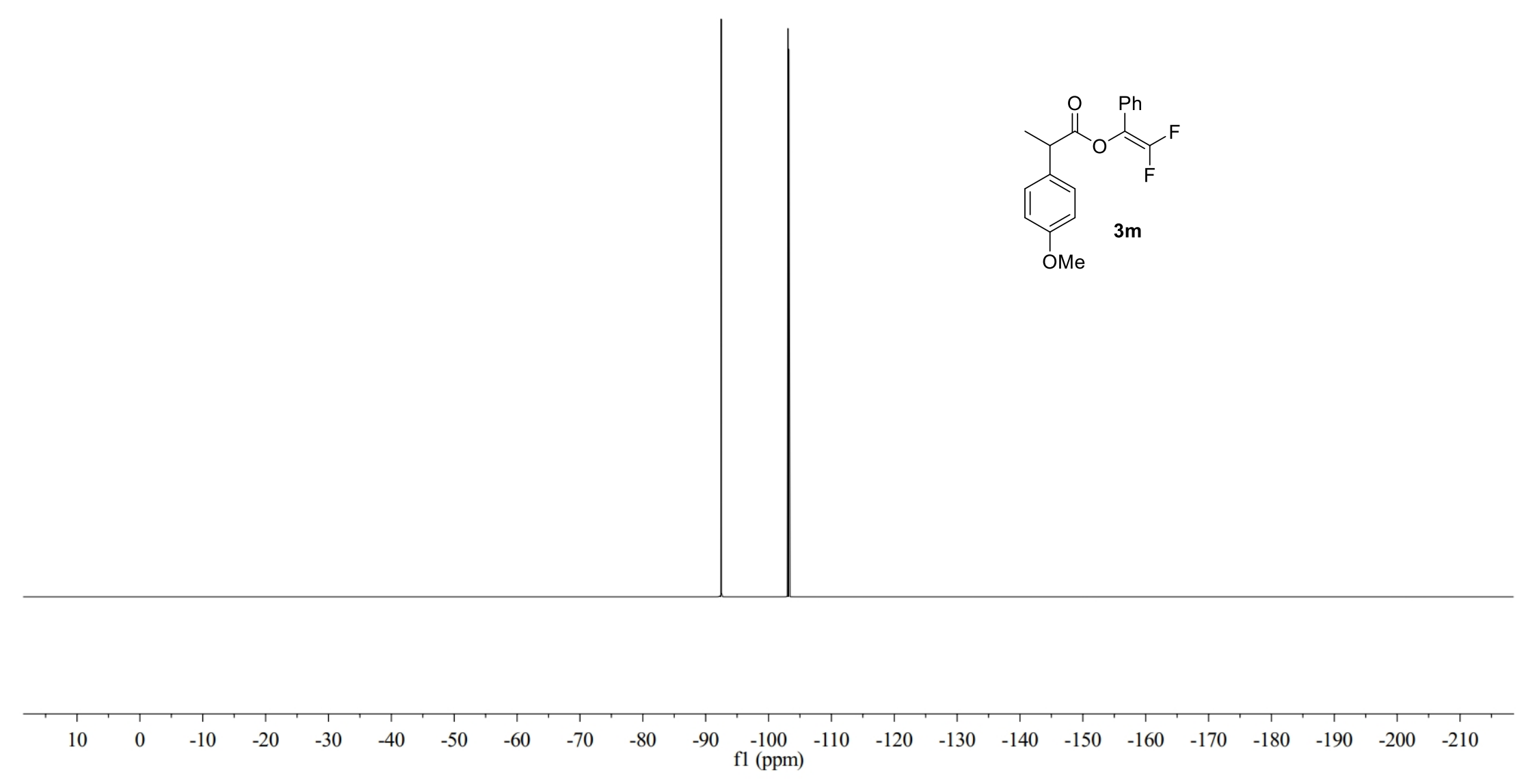

${ }^{19} \mathrm{~F}$ NMR of Compound 3m (376 MHz, $\left.\mathrm{CDCl}_{3}\right)$ 


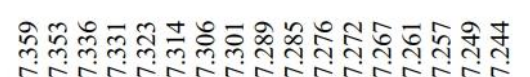

i)

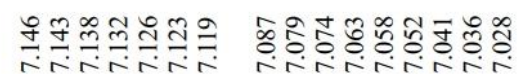

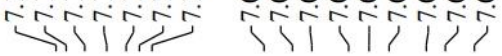

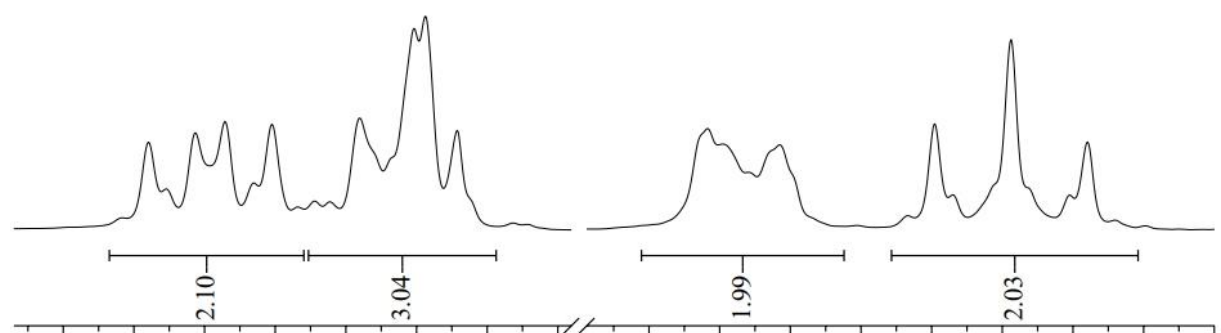

$\begin{array}{lllllllllllllllll}7.36 & 7.34 & 7.32 & 7.30 & 7.28 & 7.26 & 7.24 & 7.22 & 7.16 & 7.14 & 7.12 & 7.10 & 7.08 & 7.06 & 7.04 & 7.02 & 7.00\end{array}$ $\mathrm{f} 1$ (ppm)
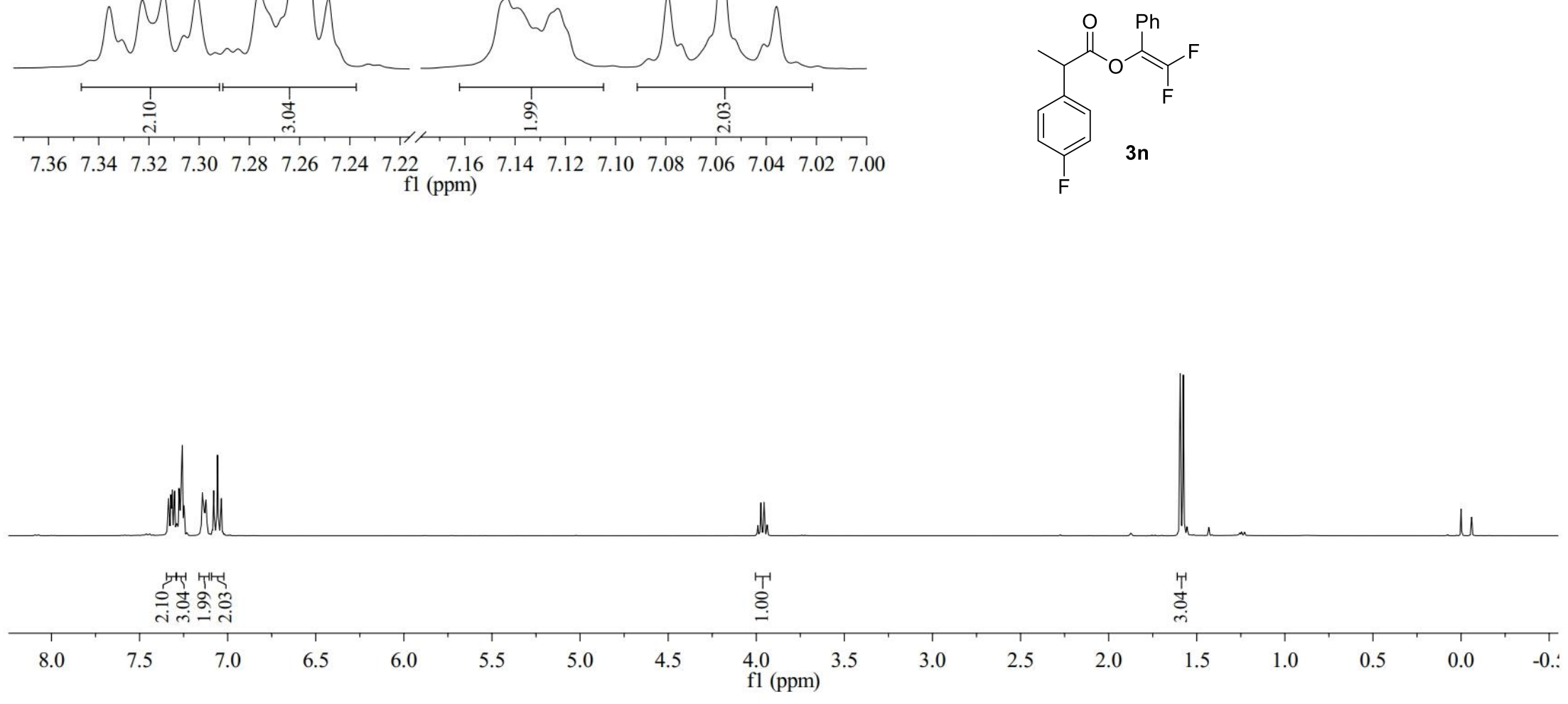

${ }^{1} \mathrm{H} \mathrm{NMR}$ of Compound 3n (400 MHz, $\left.\mathrm{CDCl}_{3}\right)$ 


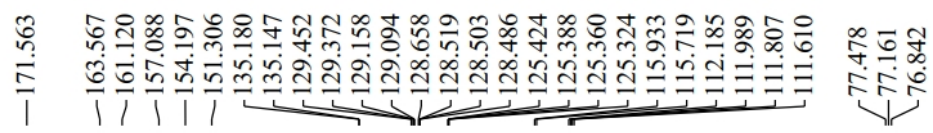

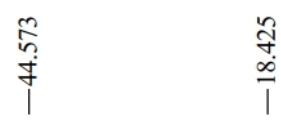
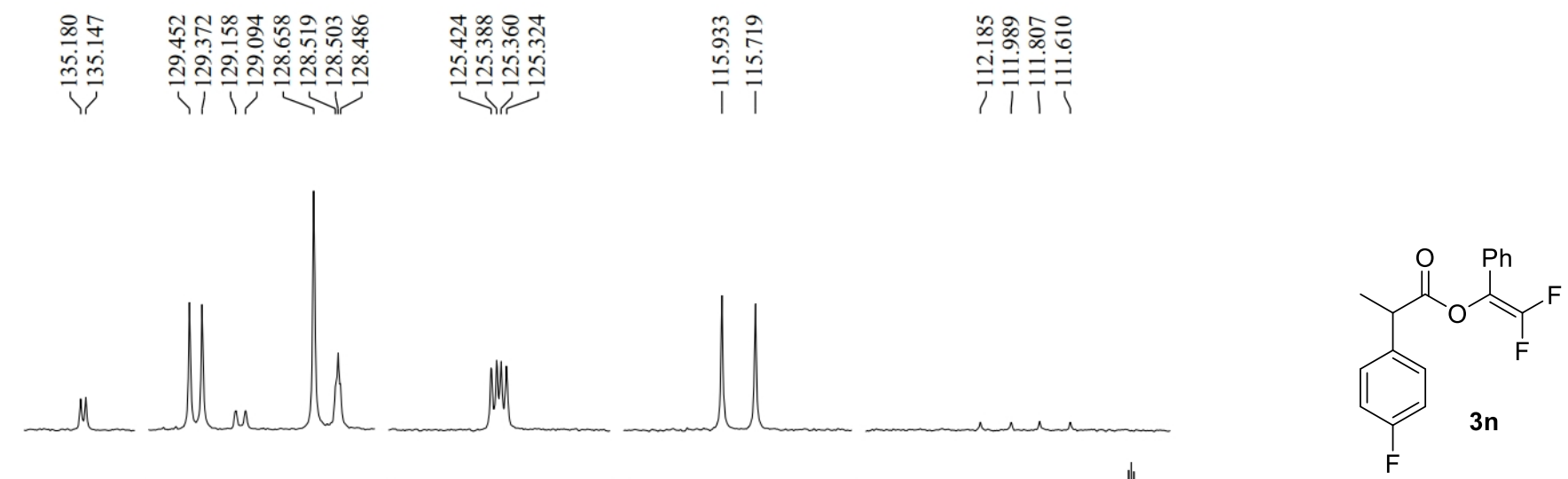

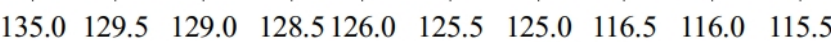
fi (ppm)

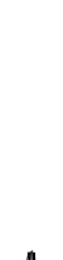

${ }^{13} \mathrm{C}$ NMR of Compound 3n (100 MHz, $\left.\mathrm{CDCl}_{3}\right)$ 


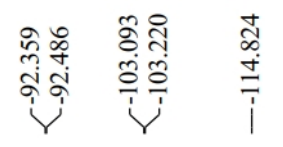

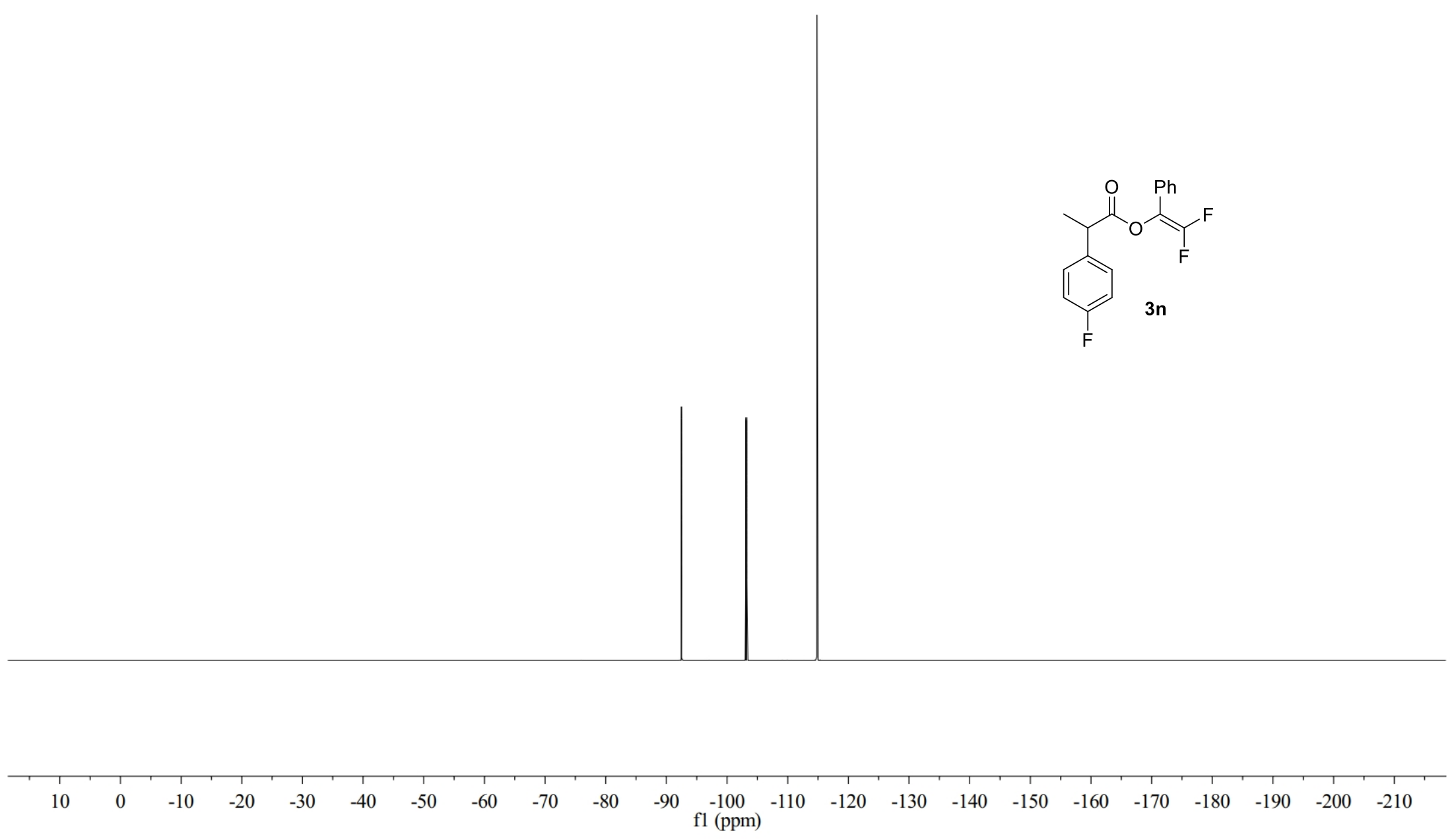

${ }^{19} \mathrm{~F}$ NMR of Compound 3n $\left(376 \mathrm{MHz}, \mathrm{CDCl}_{3}\right)$ 


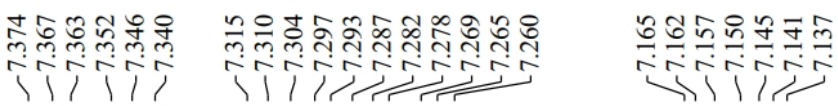

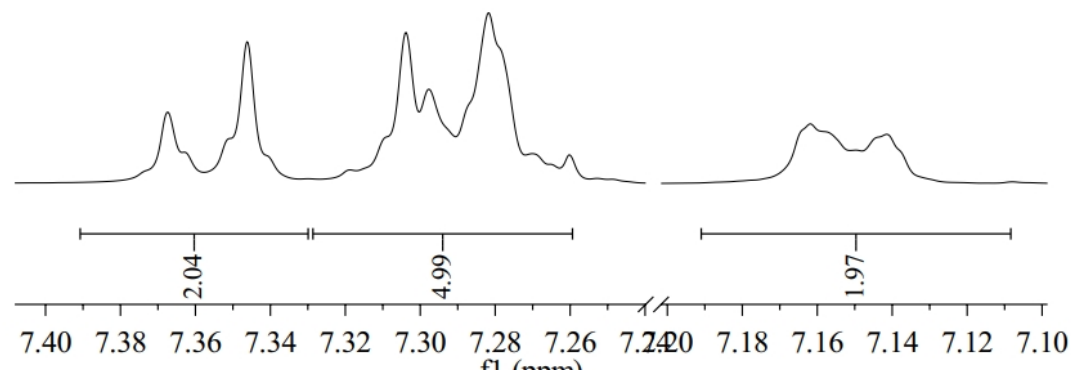

f1 (ppm)
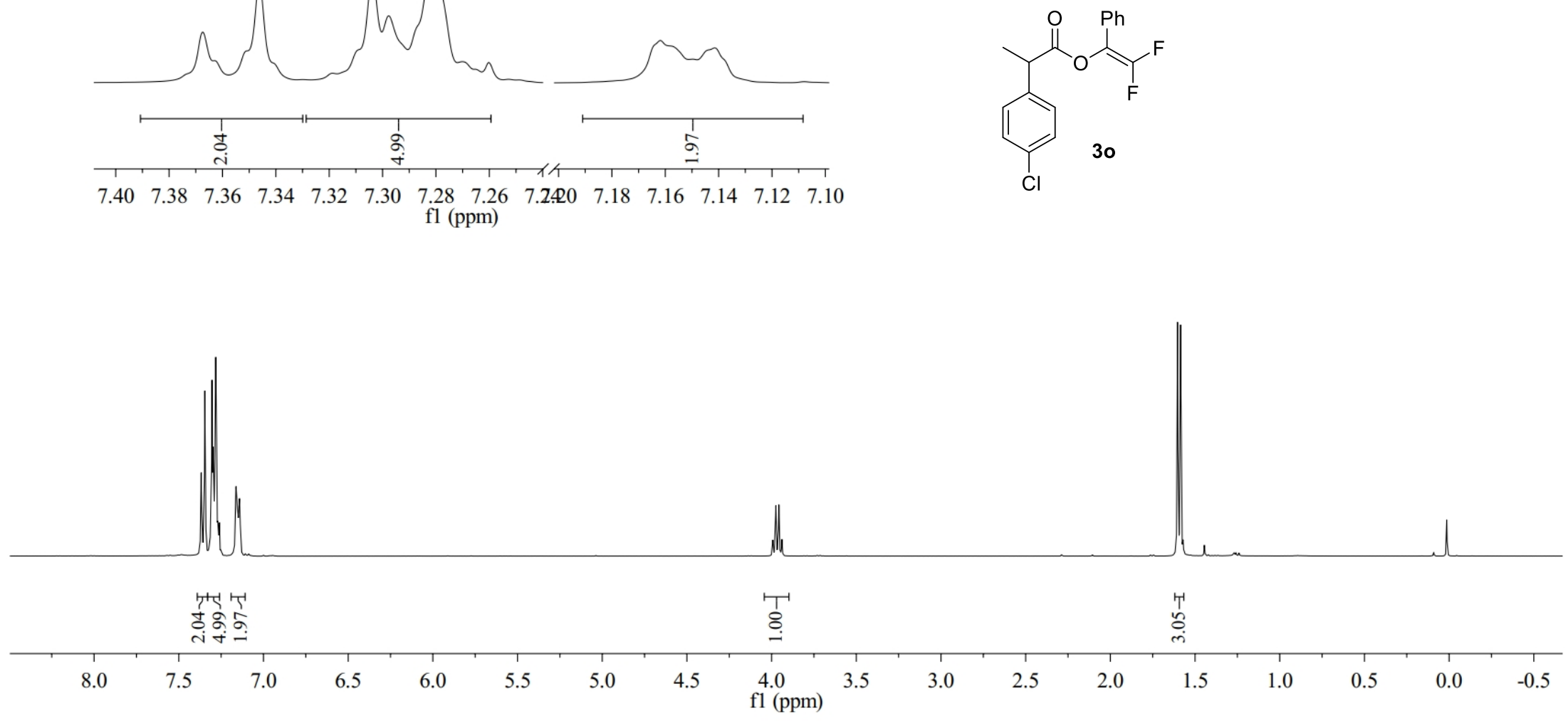

${ }^{1} \mathrm{H}$ NMR of Compound $30\left(400 \mathrm{MHz}, \mathrm{CDCl}_{3}\right)$ 

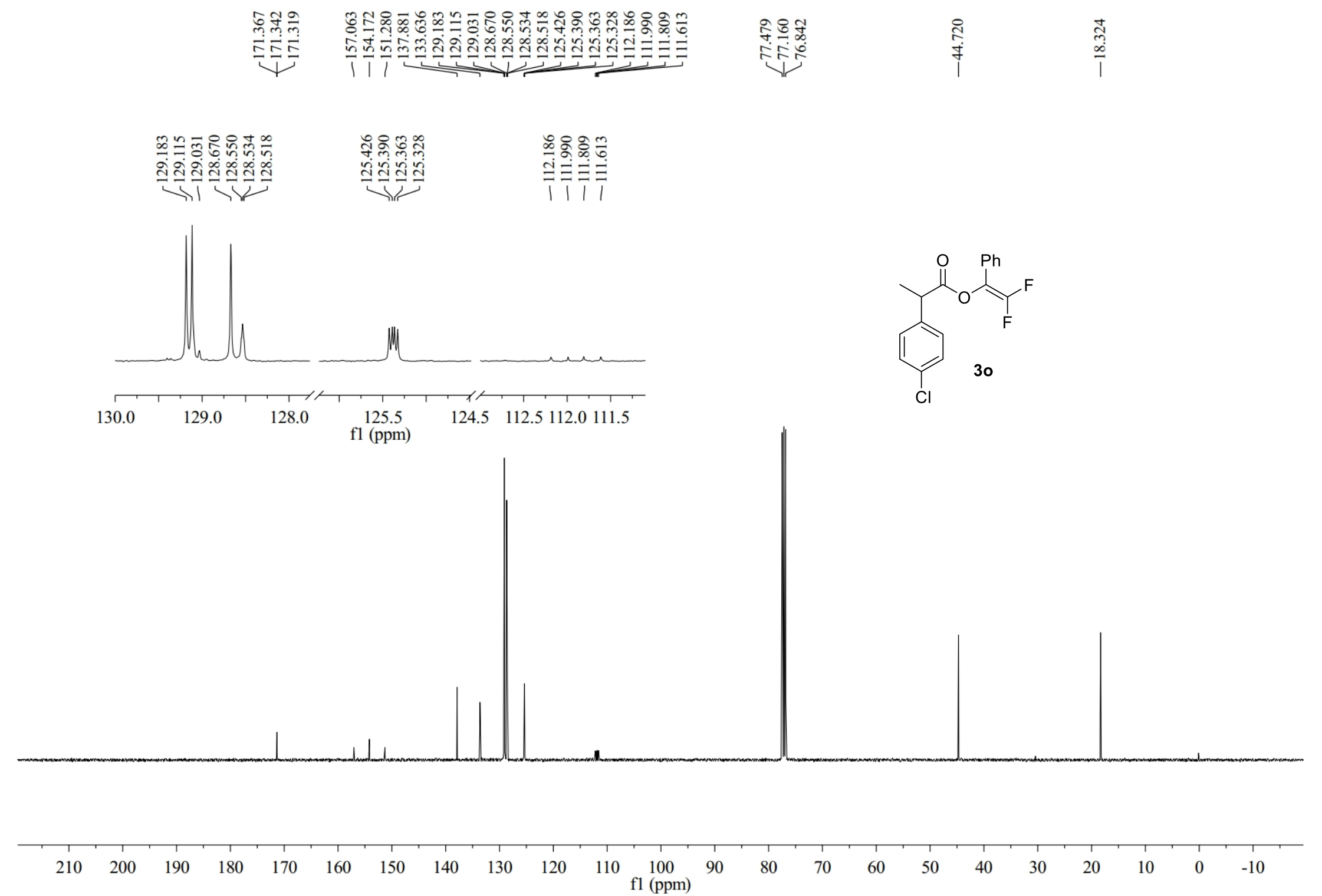

${ }^{13} \mathrm{C}$ NMR of Compound $3 \mathrm{o}\left(100 \mathrm{MHz}, \mathrm{CDCl}_{3}\right)$ 


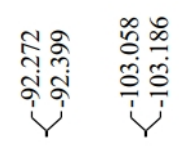
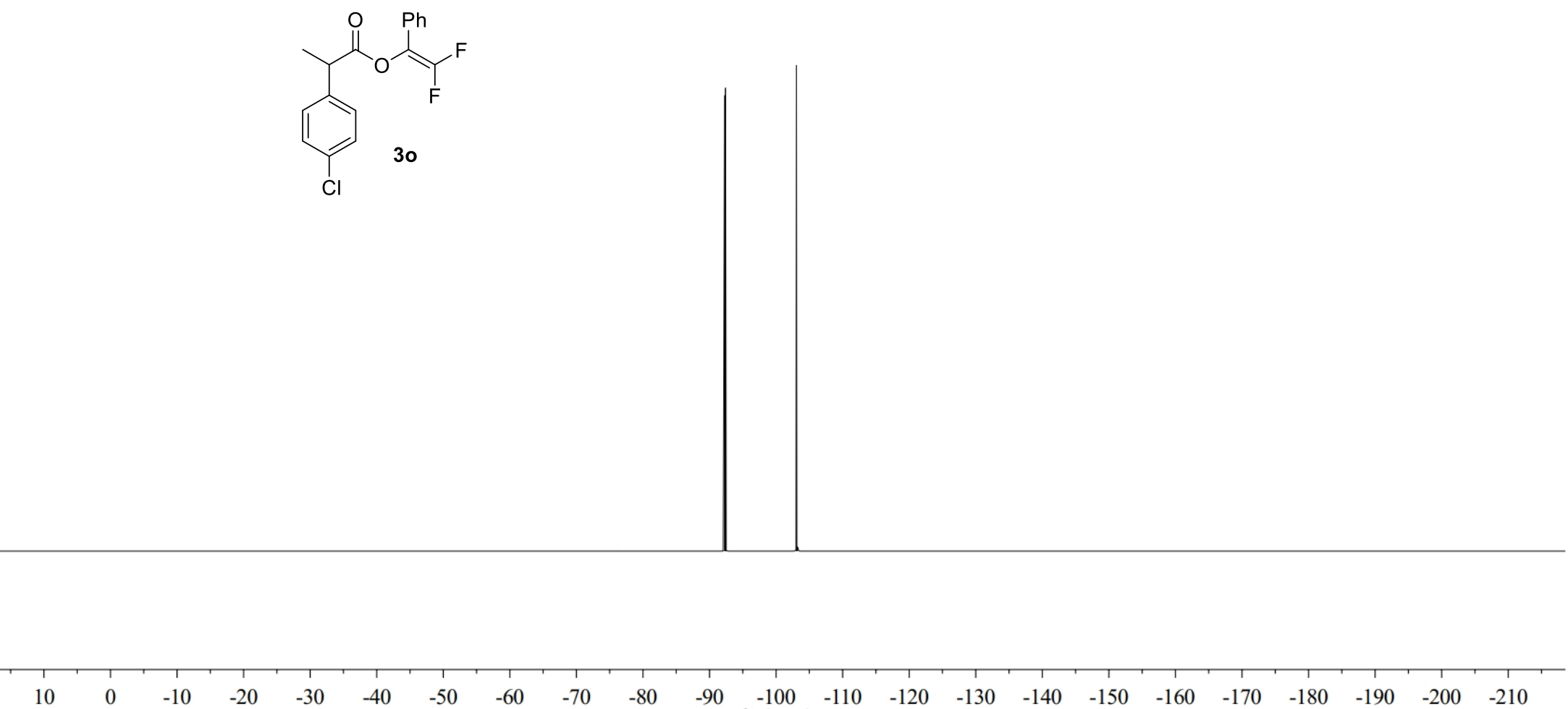

$-90-100$

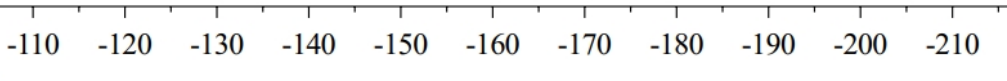

${ }^{19} \mathrm{~F}$ NMR of Compound $30\left(376 \mathrm{MHz}, \mathrm{CDCl}_{3}\right)$ 
ilit
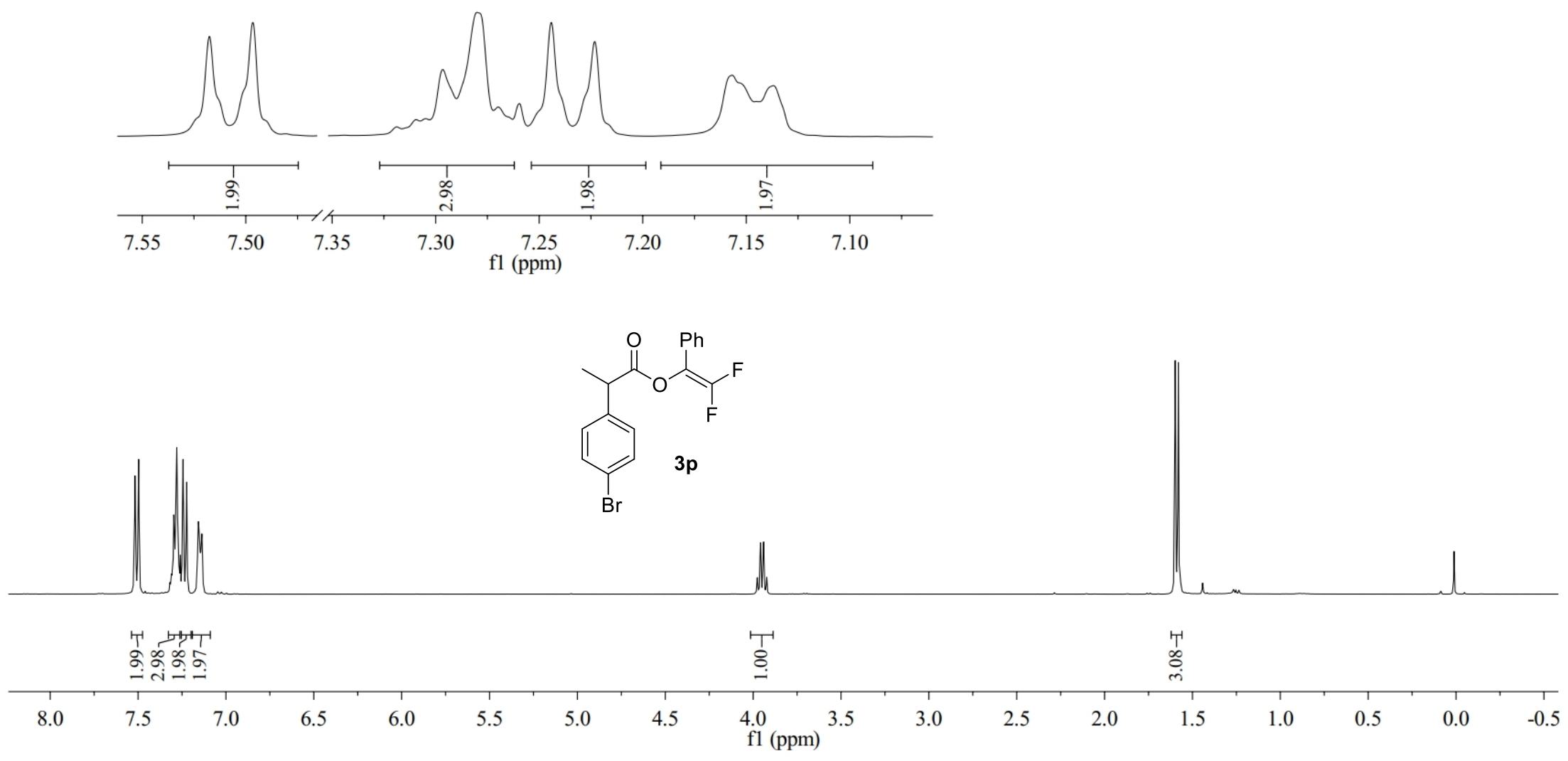

${ }^{1} \mathrm{H}$ NMR of Compound $\mathbf{3 p}\left(400 \mathrm{MHz}, \mathrm{CDCl}_{3}\right)$ 

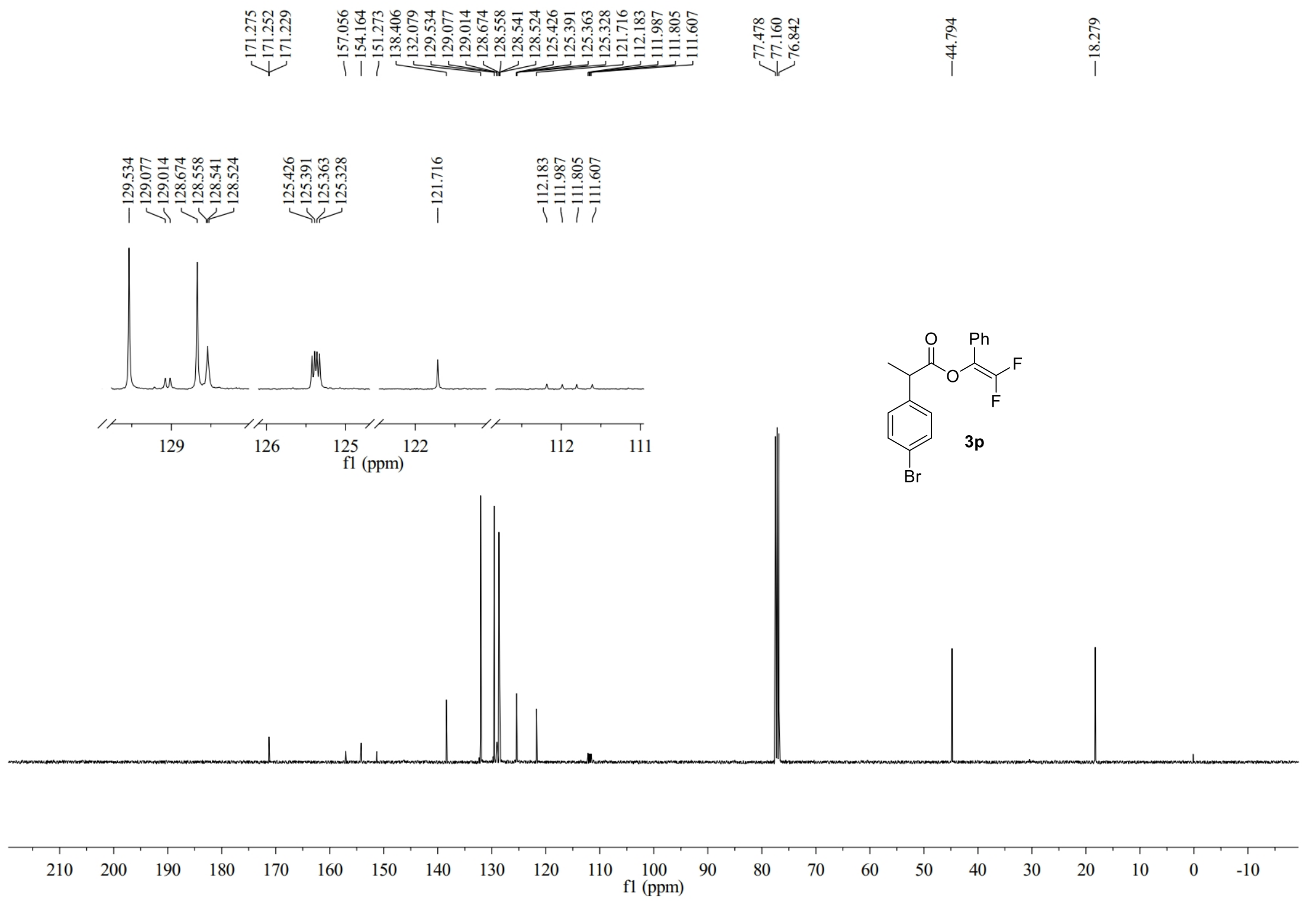

${ }^{13} \mathrm{C} \mathrm{NMR}$ of Compound 3p (100 MHz, $\left.\mathrm{CDCl}_{3}\right)$ 

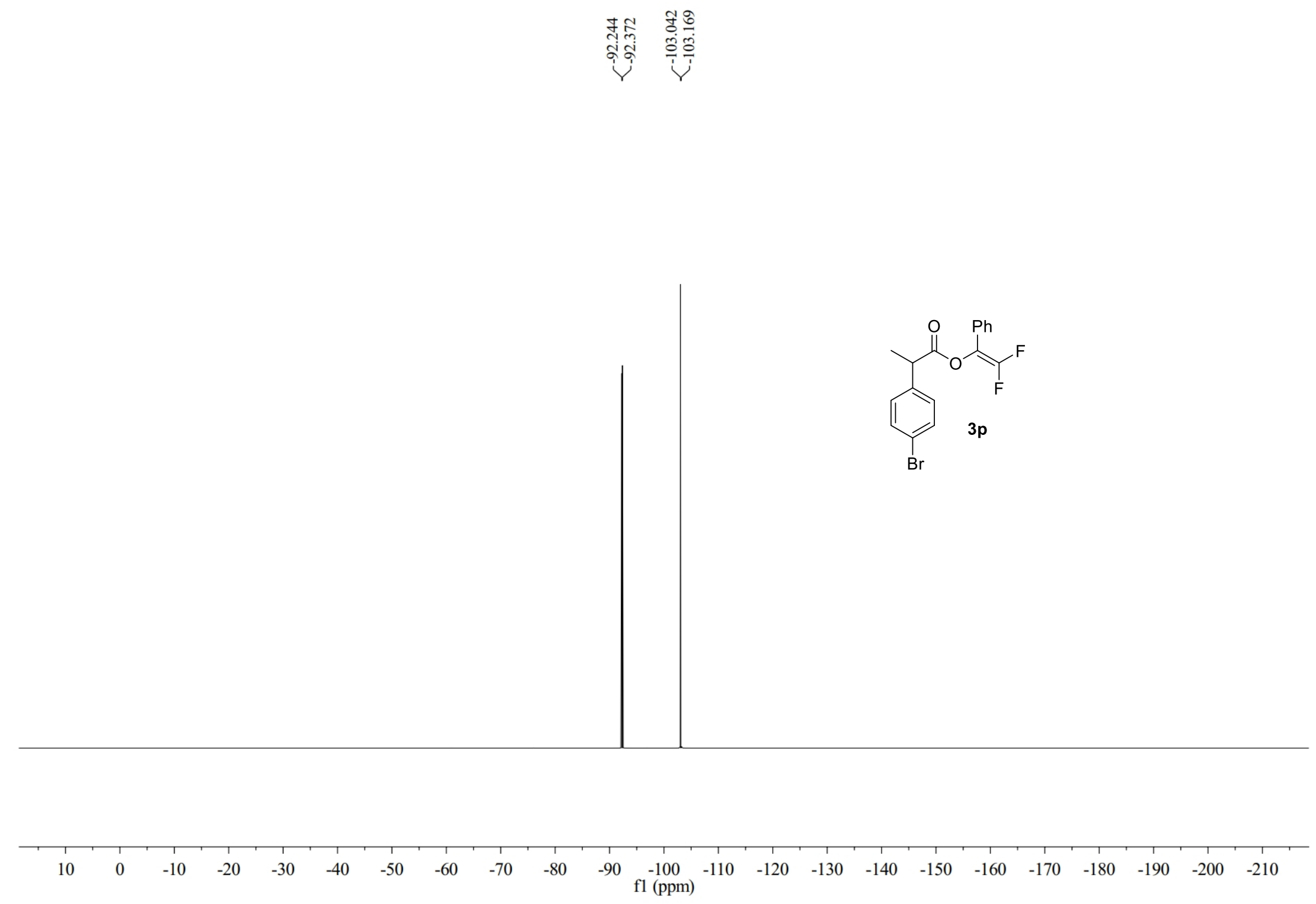

${ }^{19} \mathrm{~F}$ NMR of Compound 3p $\left(376 \mathrm{MHz}, \mathrm{CDCl}_{3}\right)$ 


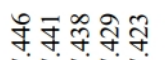

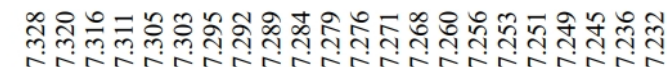

१री

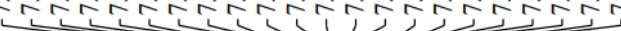
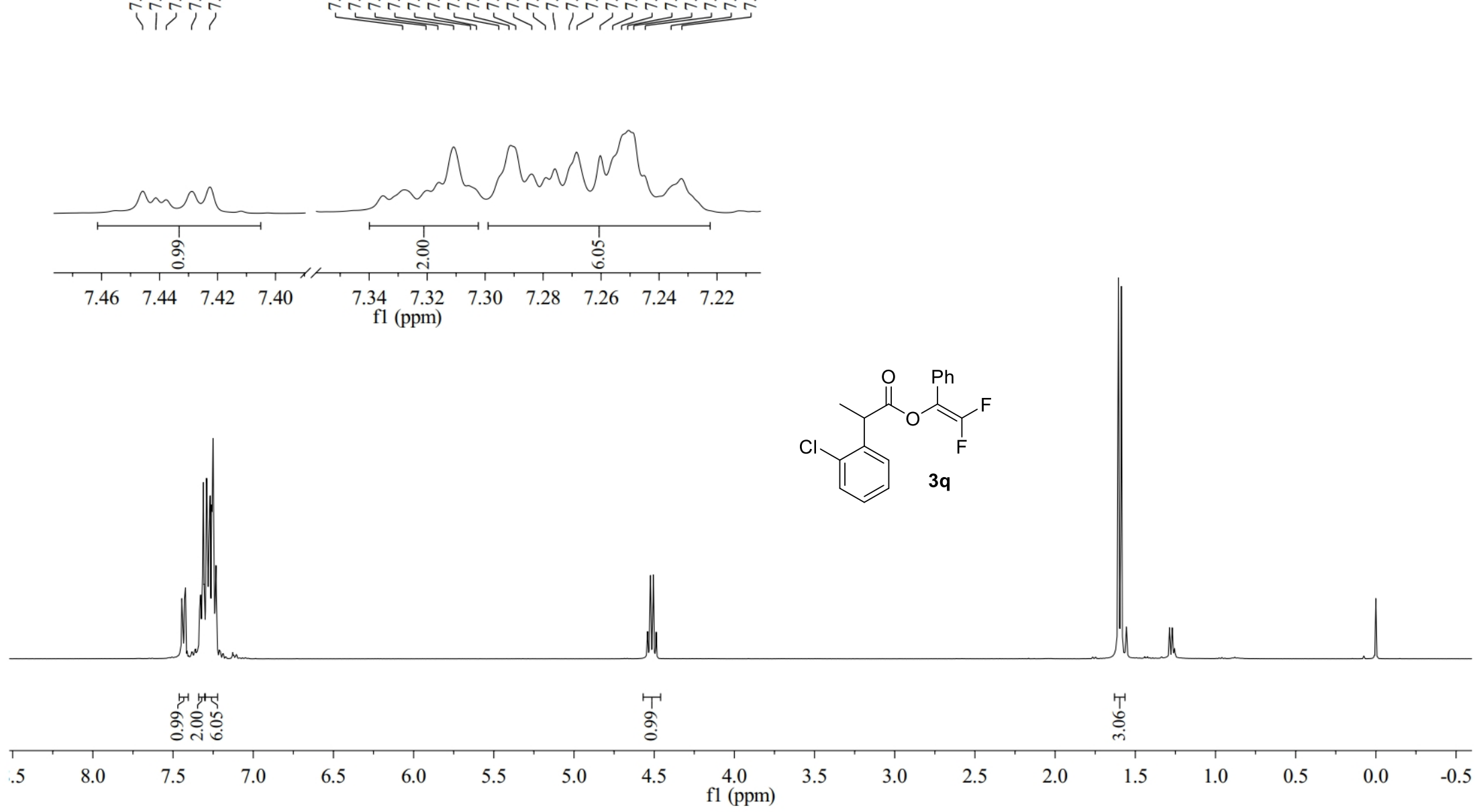

${ }^{1} \mathrm{H}$ NMR of Compound 3q (400 MHz, $\mathrm{CDCl}_{3}$ ) 


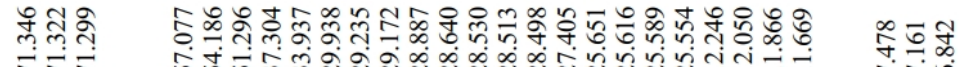 \\ 초}

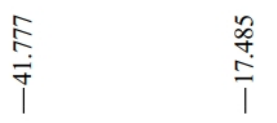

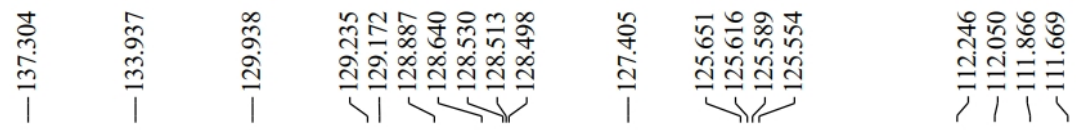
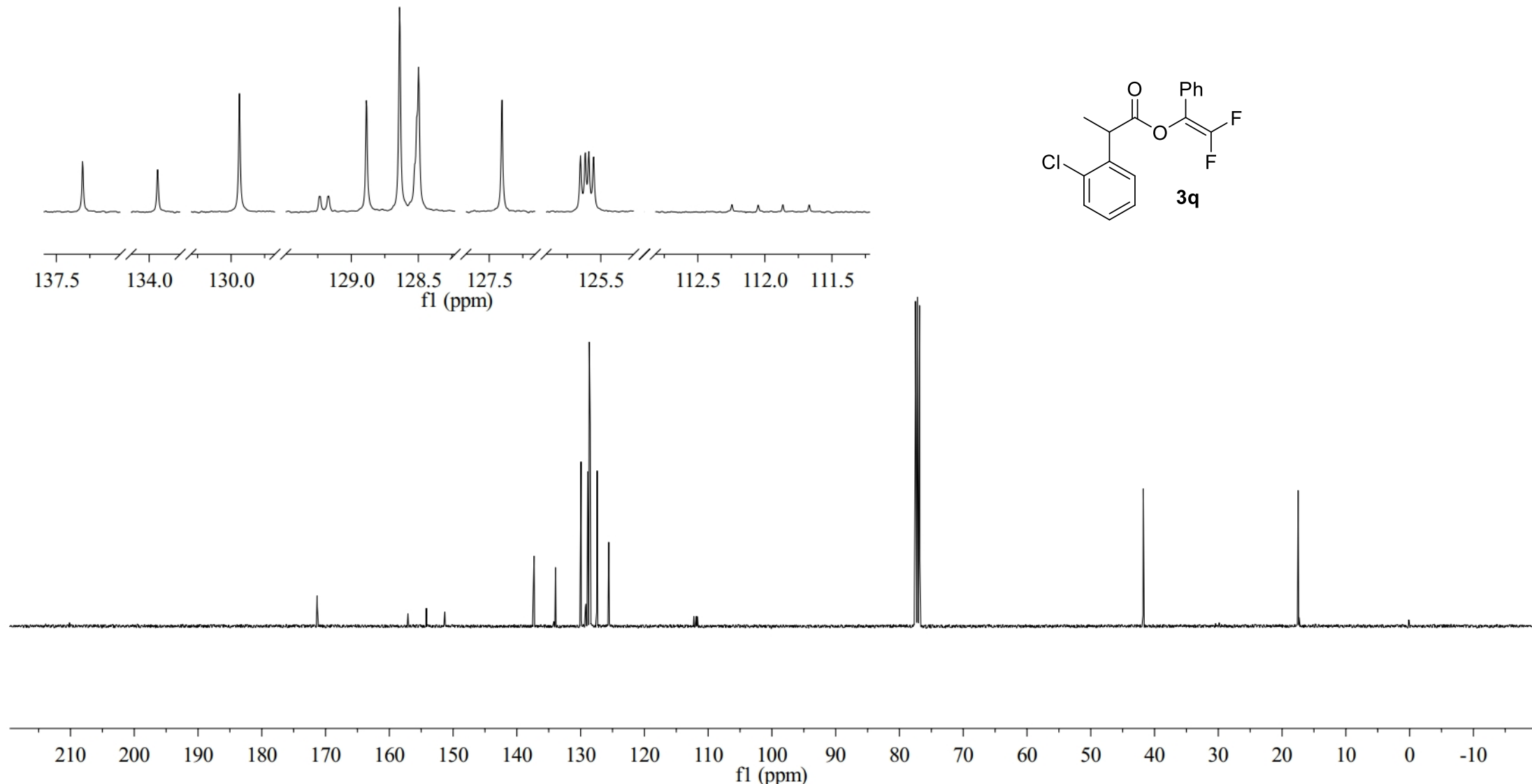

${ }^{13} \mathrm{C}$ NMR of Compound 3q $\left(100 \mathrm{MHz}, \mathrm{CDCl}_{3}\right)$ 


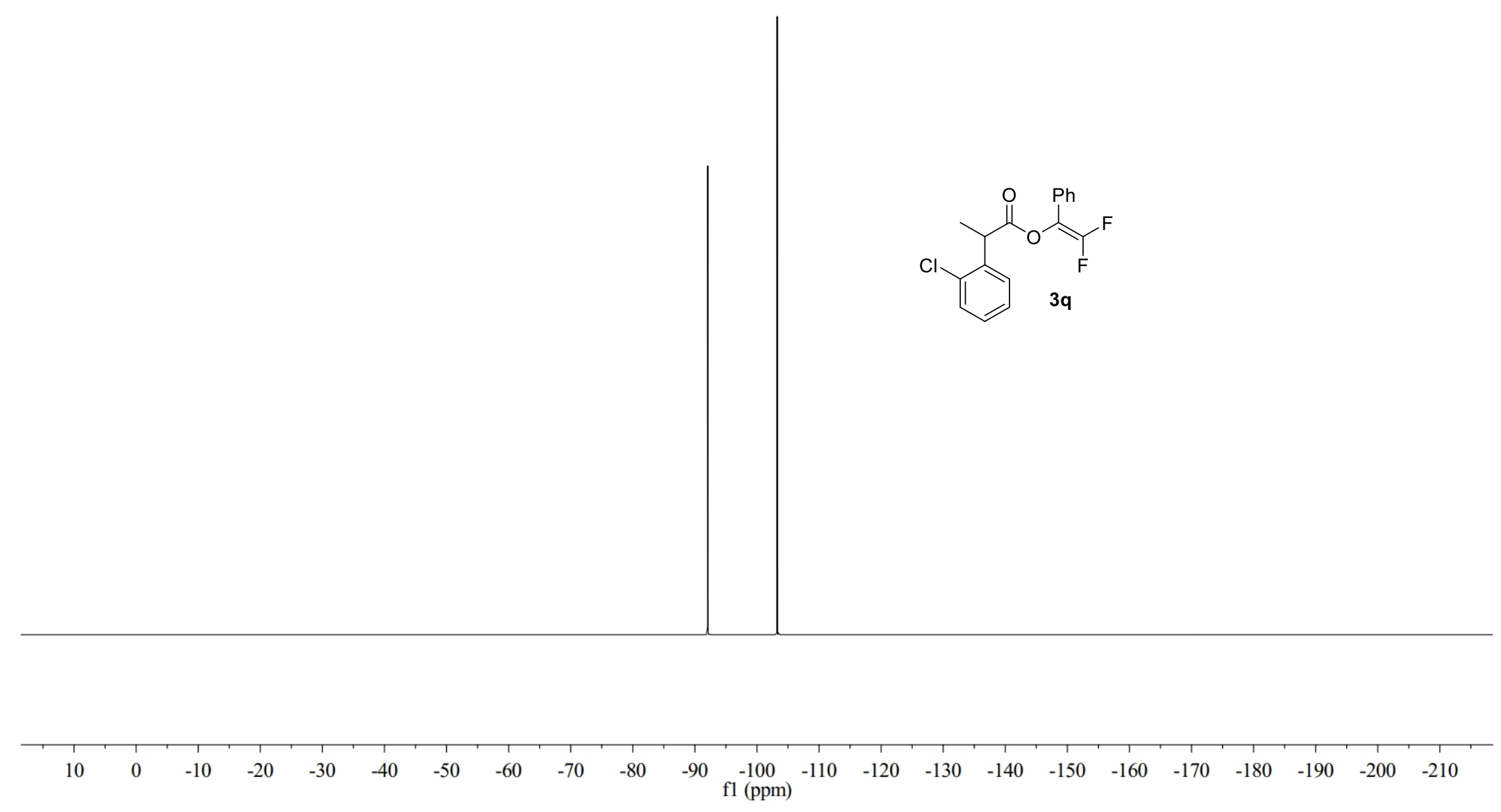

${ }^{19} \mathrm{~F}$ NMR of Compound 3q (376 MHz, $\left.\mathrm{CDCl}_{3}\right)$ 


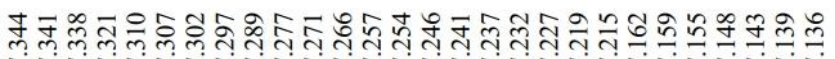

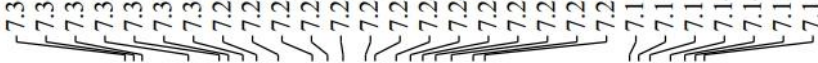
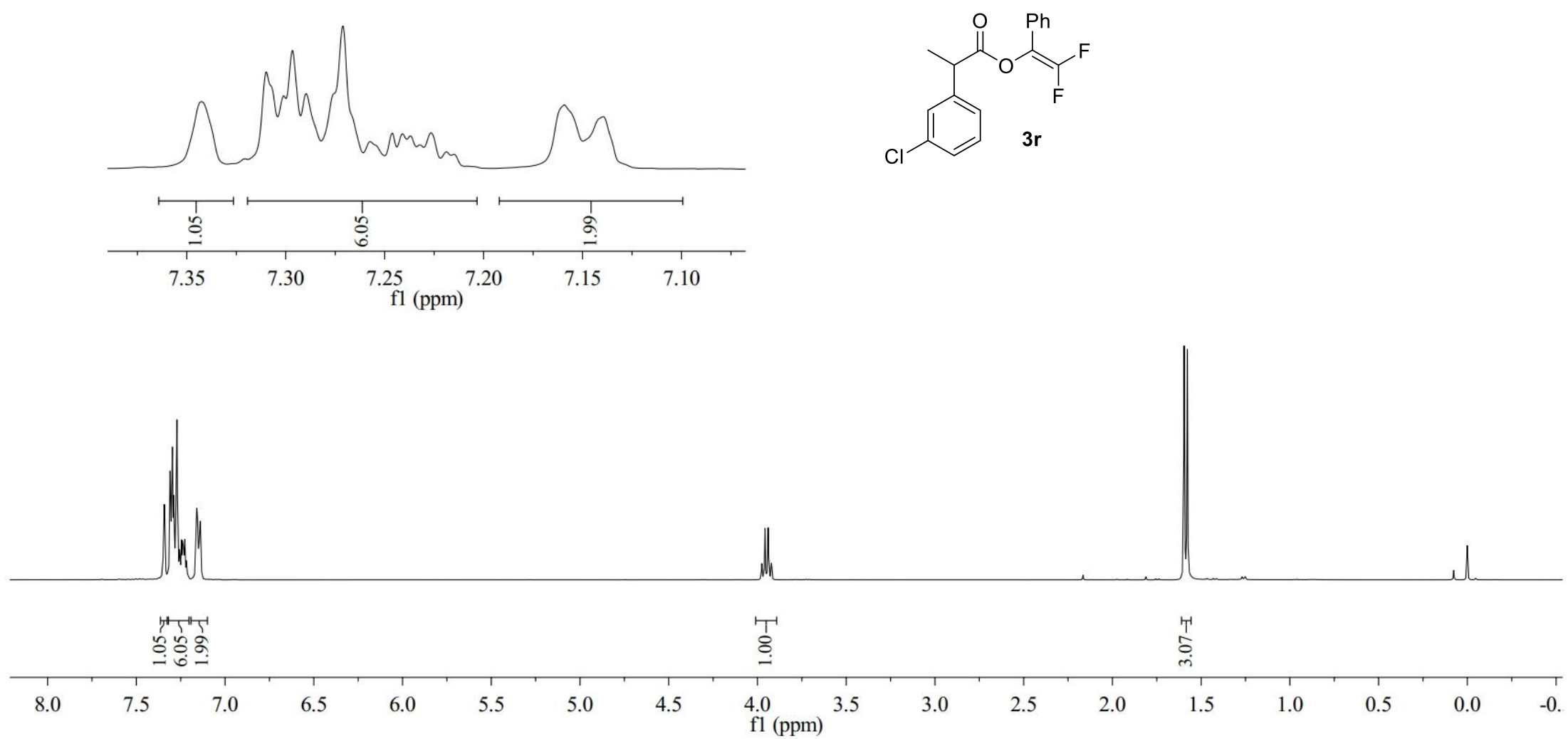

${ }^{1} \mathrm{H}$ NMR of Compound $\mathbf{3 r}\left(400 \mathrm{MHz}, \mathrm{CDCl}_{3}\right)$ 


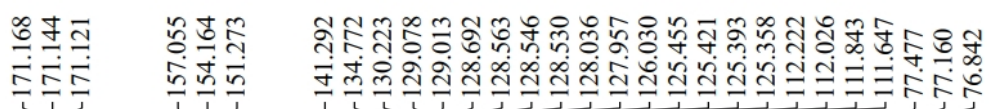

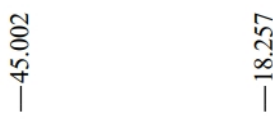

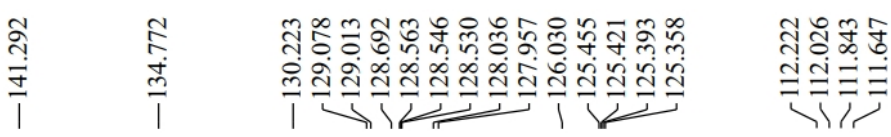
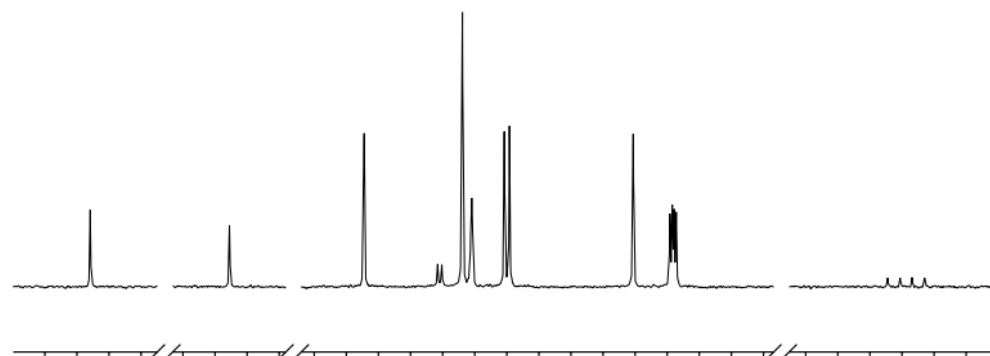

$\begin{array}{llllllllllllll}142 & 141 & 135 & 13431 & 130 & 129 & 128 & 127 & 126 & 125 & 124 & 113 & 112 & 111\end{array}$
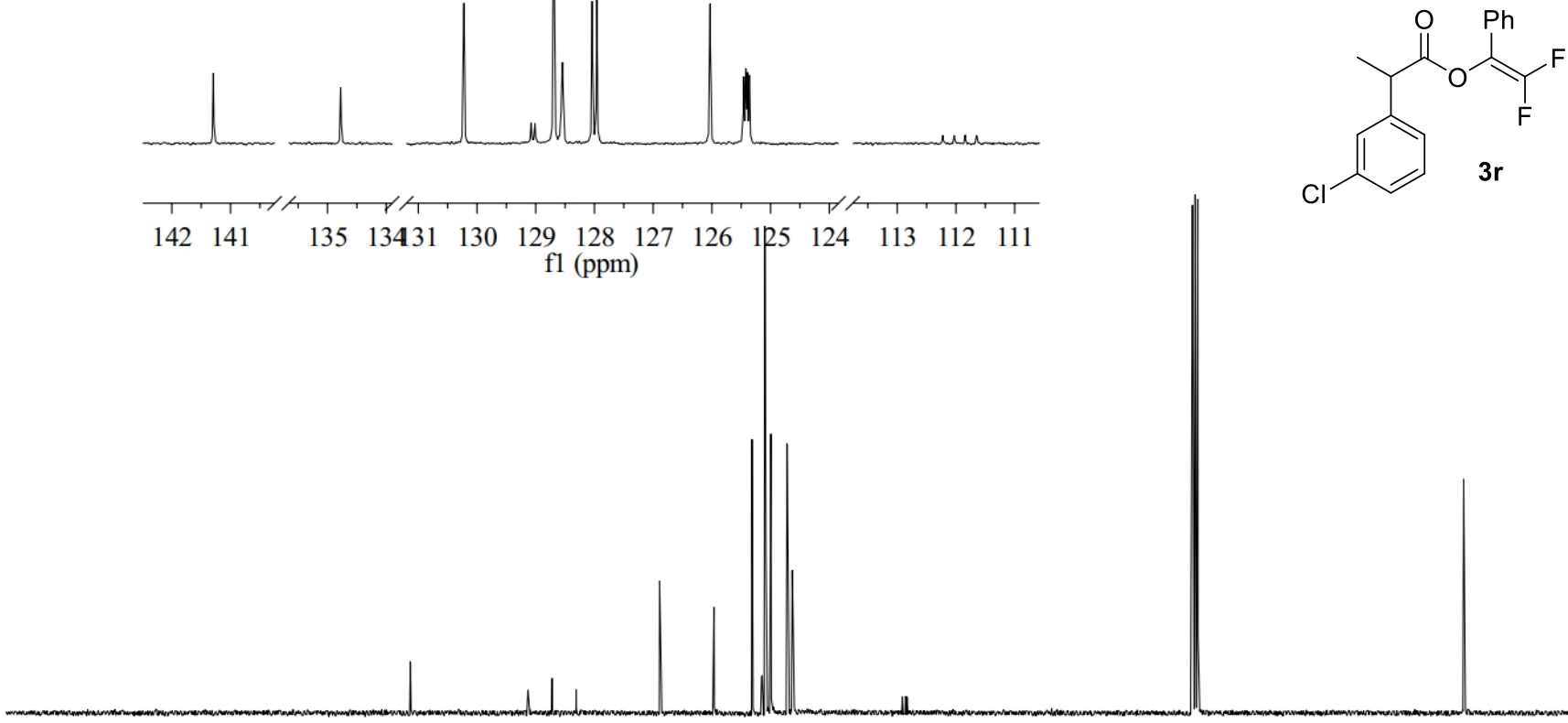

$\mathrm{Cl}$

$3 r$

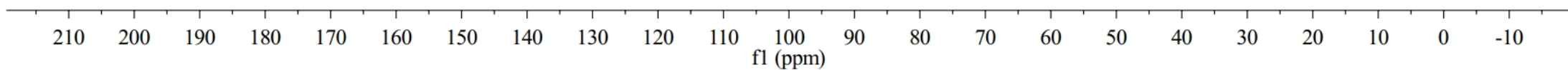

${ }^{13} \mathrm{C}$ NMR of Compound $\mathbf{3 r}\left(100 \mathrm{MHz}, \mathrm{CDCl}_{3}\right)$ 


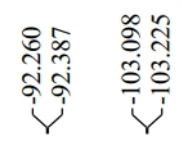

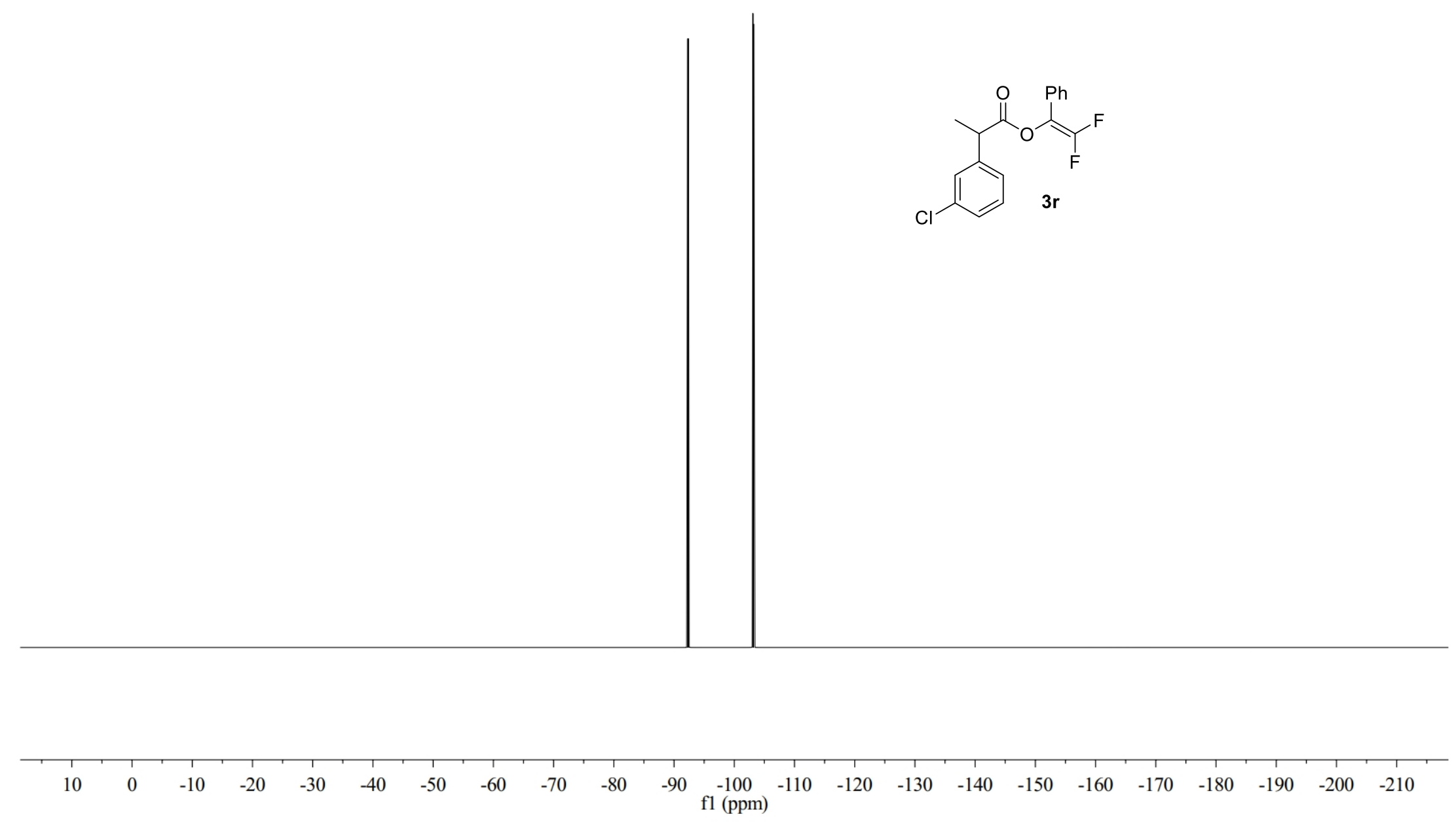

${ }^{19} \mathrm{~F}$ NMR of Compound $\mathbf{3 r}\left(376 \mathrm{MHz}, \mathrm{CDCl}_{3}\right)$ 


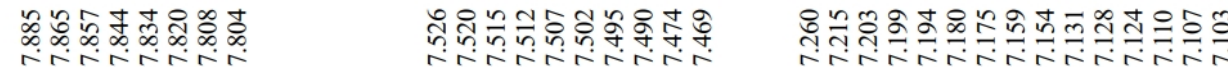

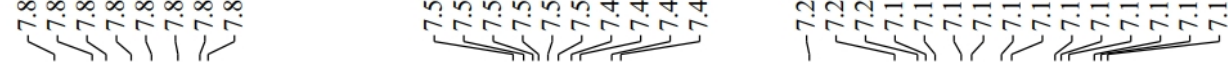
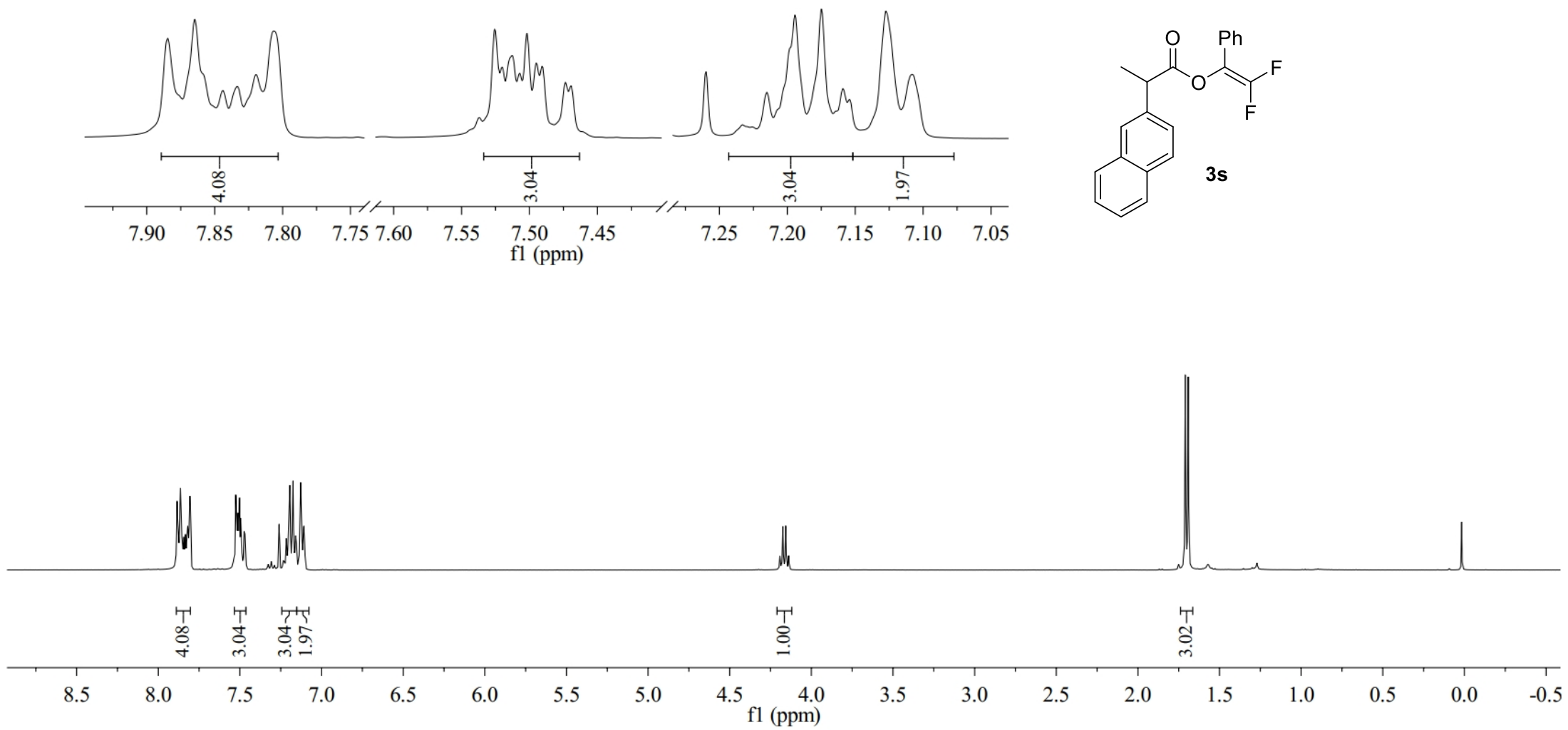

${ }^{1} \mathrm{H}$ NMR of Compound 3s (400 MHz, $\left.\mathrm{CDCl}_{3}\right)$ 


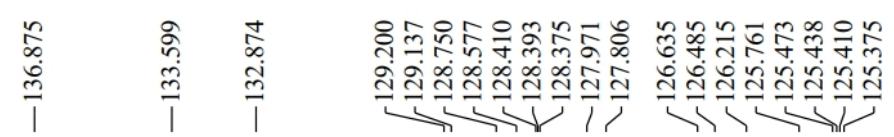
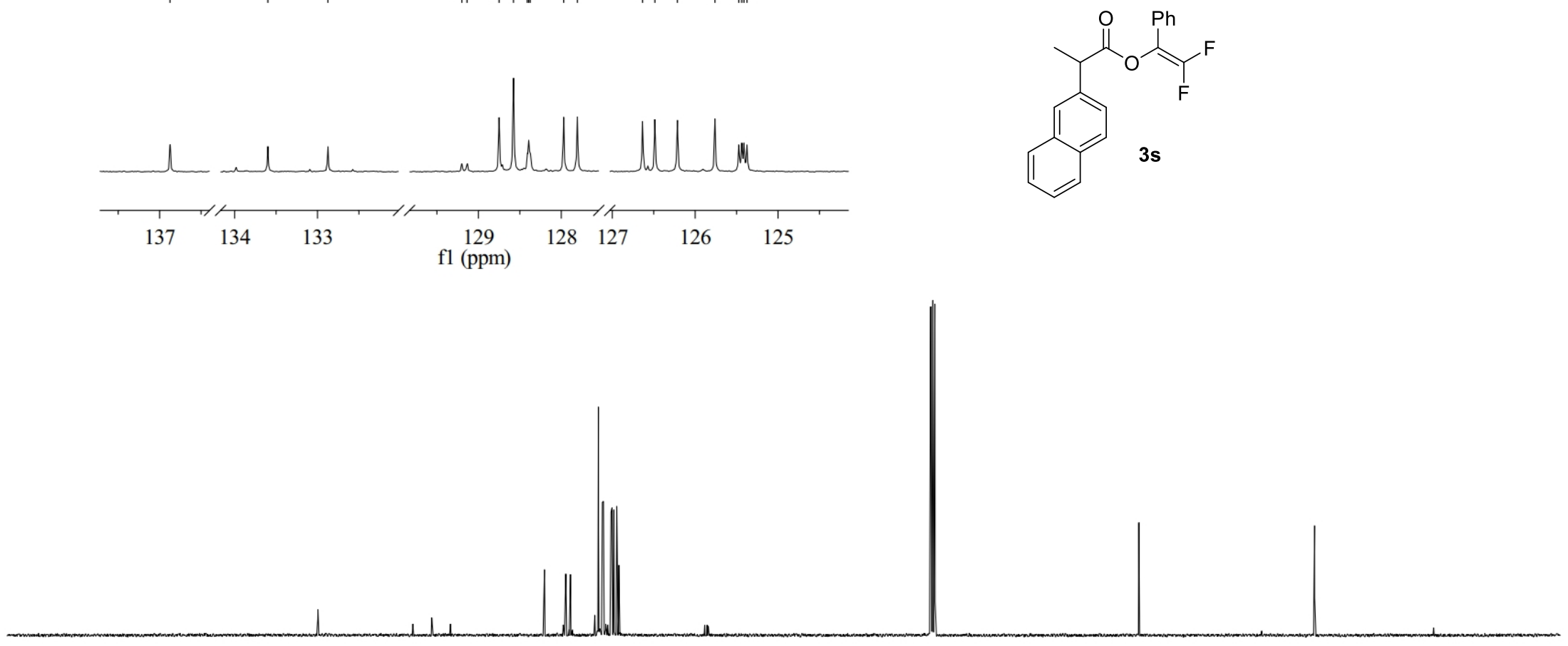

210

$200 \quad 190$

$190 \quad 180$

${ }^{13} \mathrm{C}$ NMR of Compound $3 \mathbf{s}\left(100 \mathrm{MHz}, \mathrm{CDCl}_{3}\right)$ 

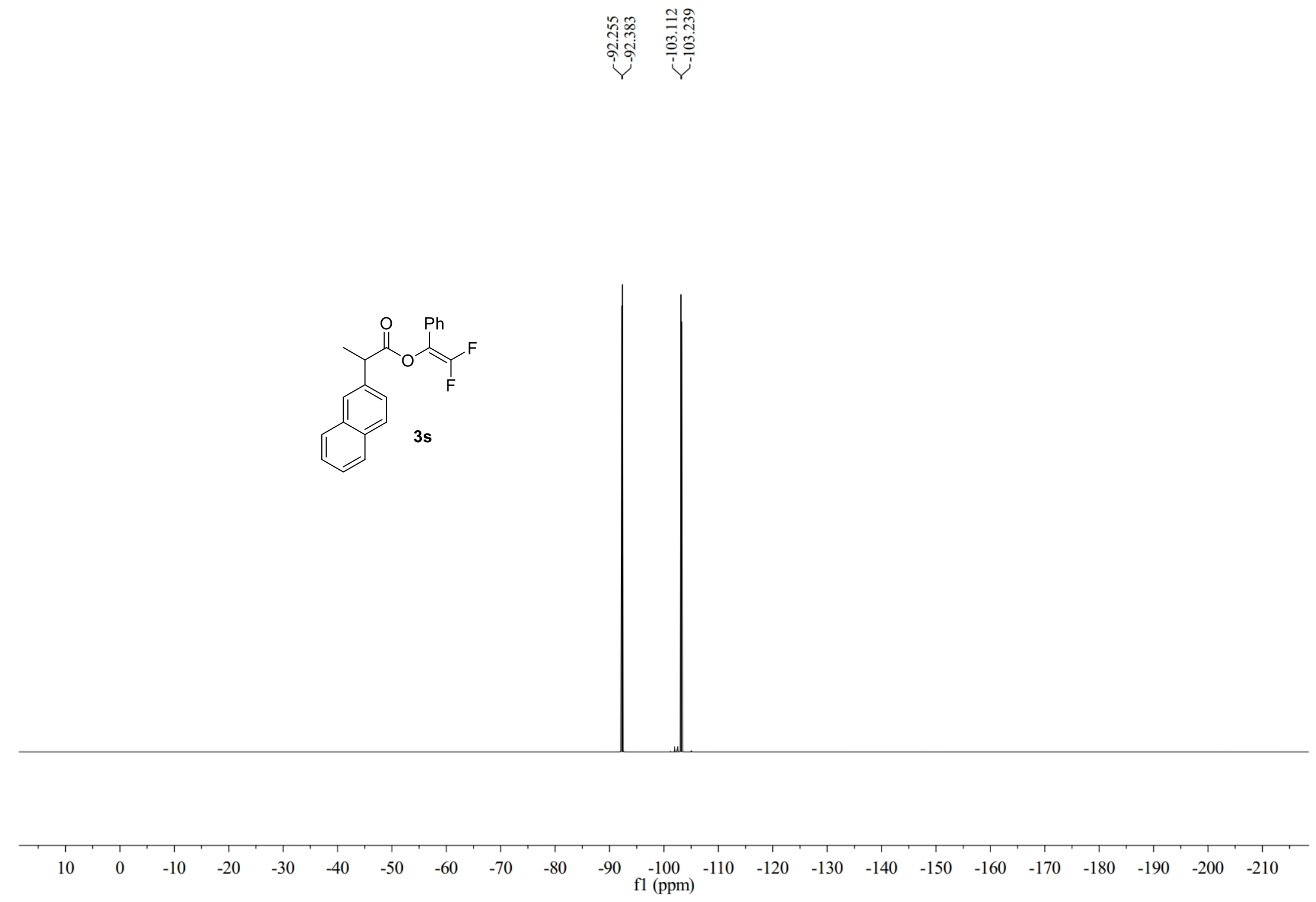

${ }^{19} \mathrm{~F}$ NMR of Compound $3 s\left(376 \mathrm{MHz}, \mathrm{CDCl}_{3}\right)$ 

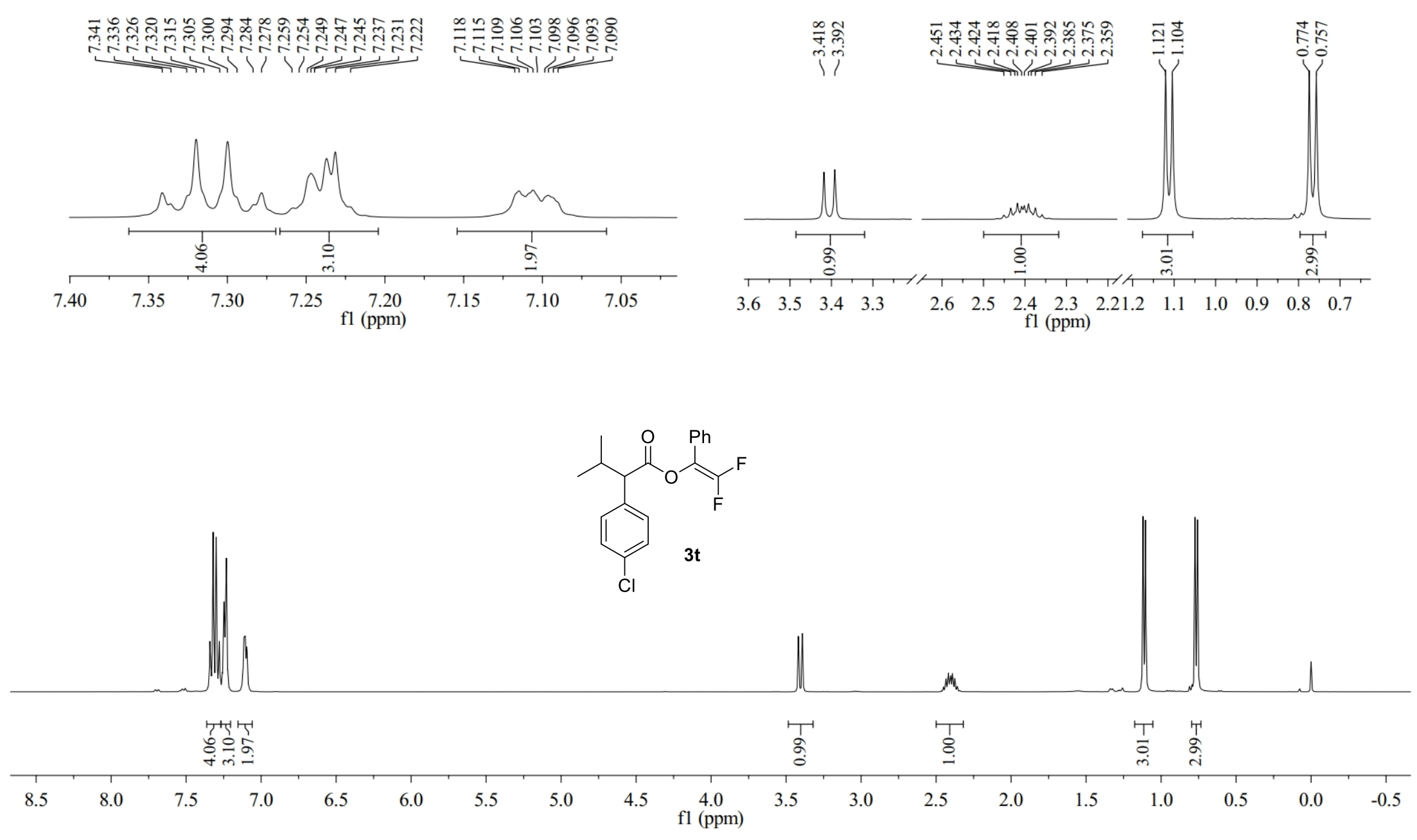

${ }^{1} \mathrm{H}$ NMR of Compound $\mathbf{3 t}\left(400 \mathrm{MHz}, \mathrm{CDCl}_{3}\right)$ 


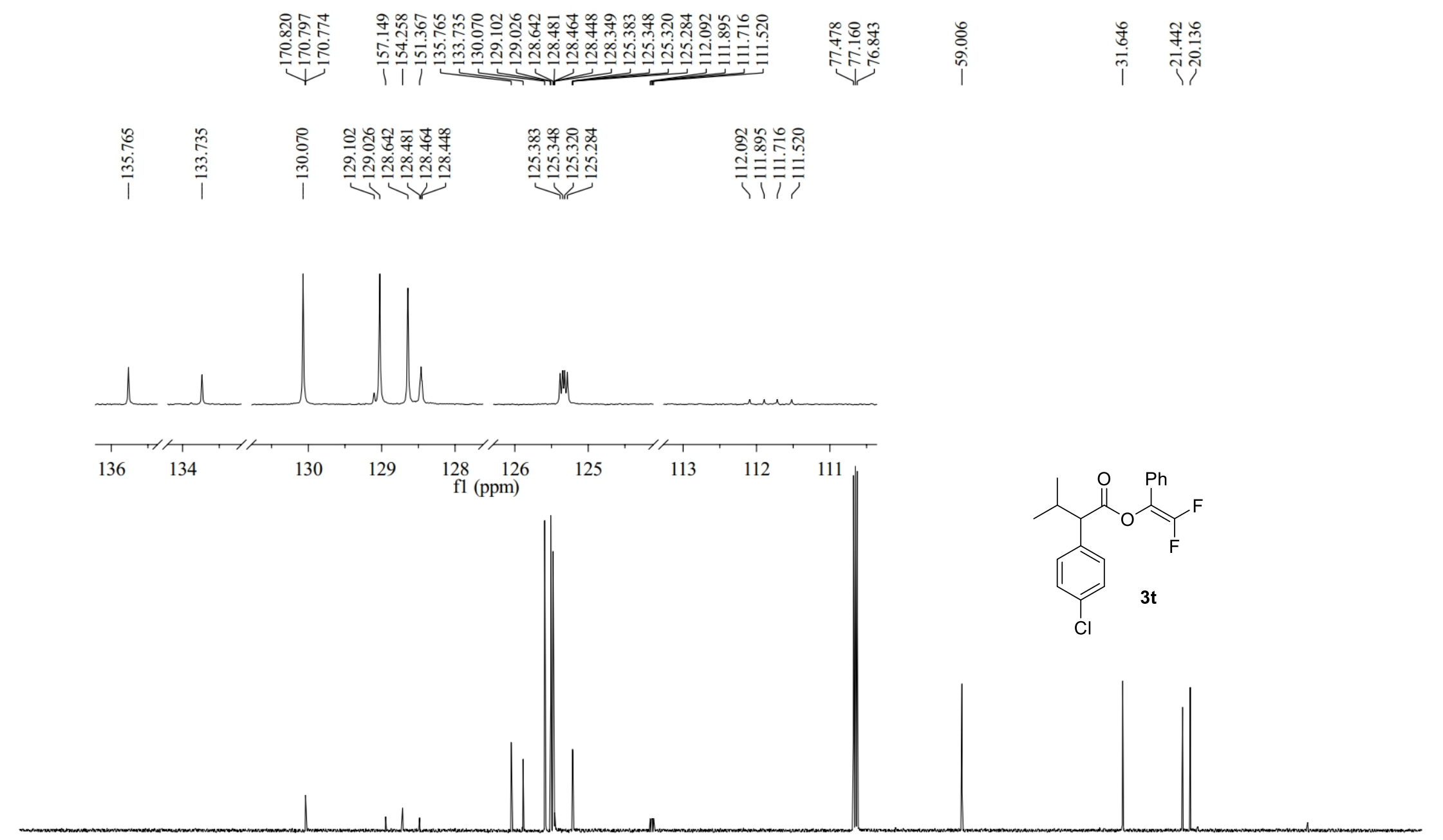

$\begin{array}{lllllllllllllllllllllllllllll}210 & 200 & 190 & 180 & 170 & 160 & 150 & 140 & 130 & 120 & 110 & \begin{array}{c}100 \\ \mathrm{fl}(\mathrm{ppm})\end{array} & 90 & 80 & 70 & 60 & 50 & 40 & 30 & 20 & 10 & 0 & -10 & \end{array}$

${ }^{13} \mathrm{C}$ NMR of Compound $3 t\left(100 \mathrm{MHz}, \mathrm{CDCl}_{3}\right)$ 


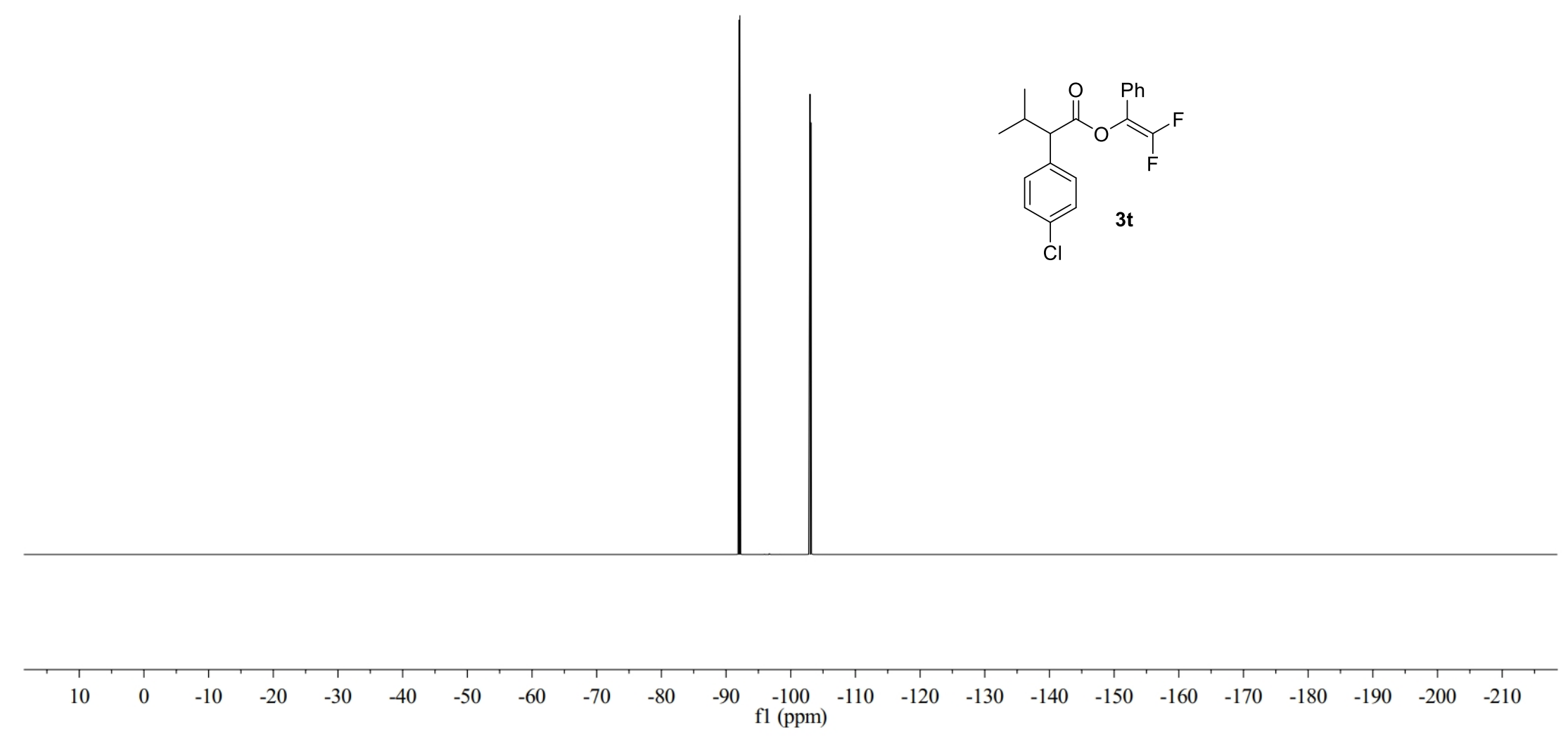

${ }^{19} \mathrm{~F}$ NMR of Compound $3 \mathrm{t}\left(376 \mathrm{MHz}, \mathrm{CDCl}_{3}\right)$ 


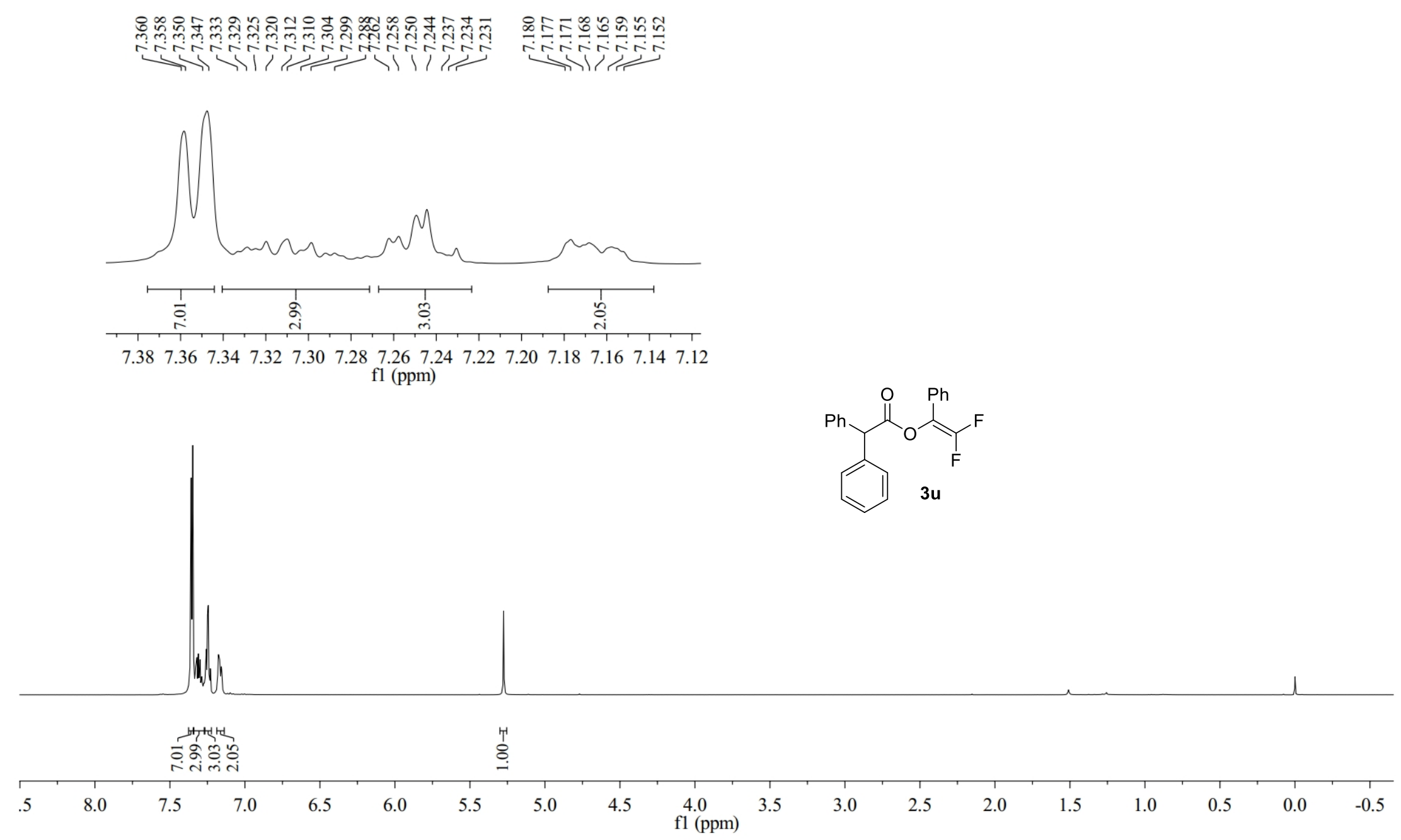

${ }^{1} \mathrm{H}$ NMR of Compound $\mathbf{3 u}\left(400 \mathrm{MHz}, \mathrm{CDCl}_{3}\right)$ 

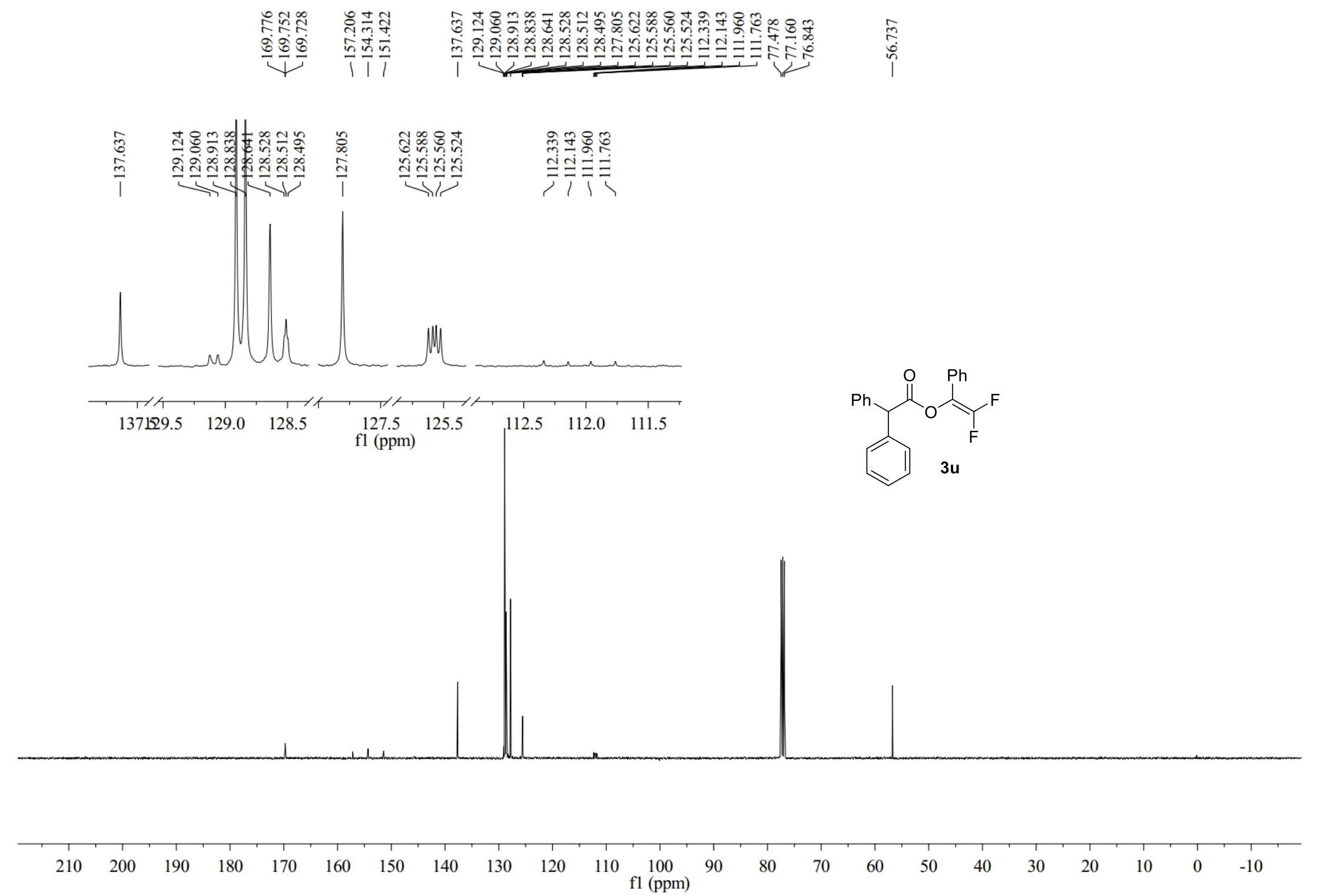

${ }^{13} \mathrm{C}$ NMR of Compound 3u $\left(100 \mathrm{MHz}, \mathrm{CDCl}_{3}\right)$ 


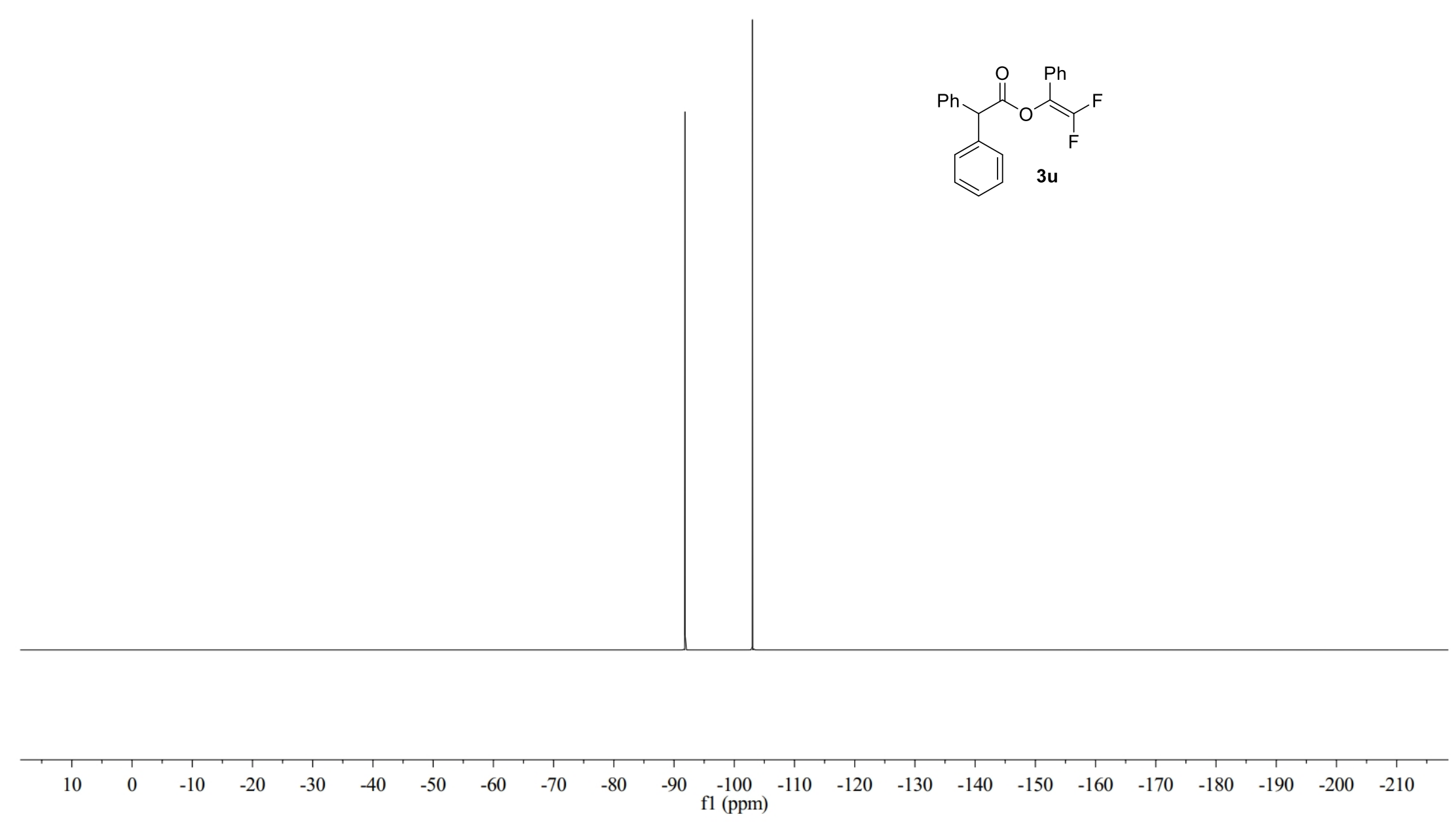

${ }^{19} \mathrm{~F}$ NMR of Compound $3 \mathbf{u}\left(376 \mathrm{MHz}, \mathrm{CDCl}_{3}\right)$ 


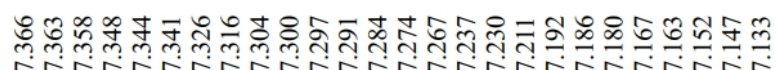

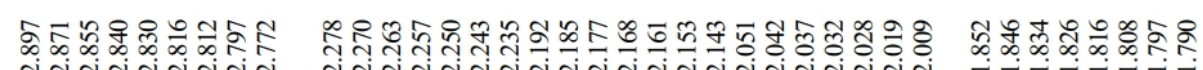

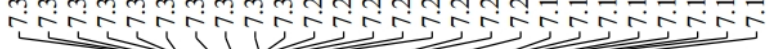
ن
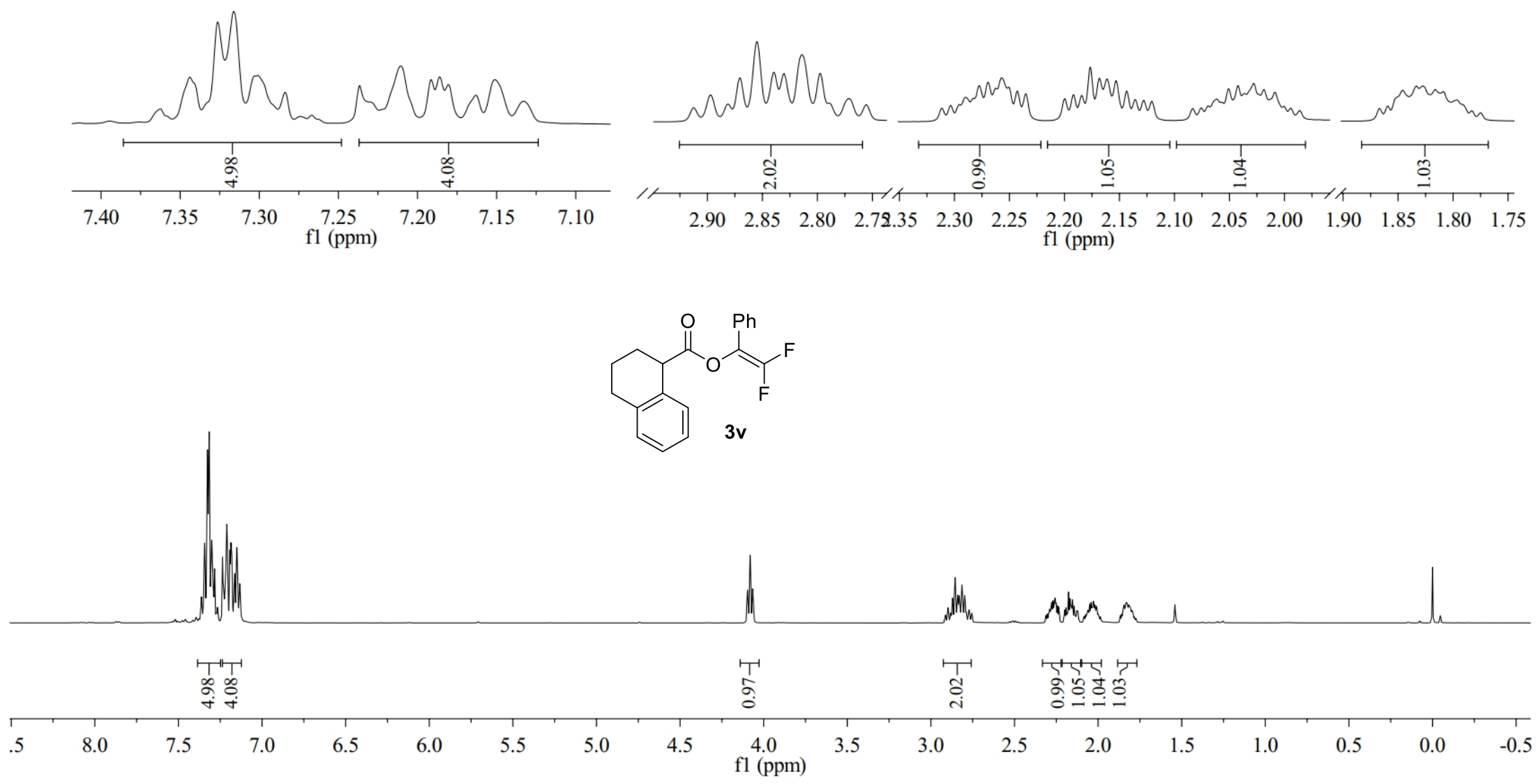

${ }^{1} \mathrm{H}$ NMR of Compound $\mathbf{3 v}\left(400 \mathrm{MHz}, \mathrm{CDCl}_{3}\right)$ 

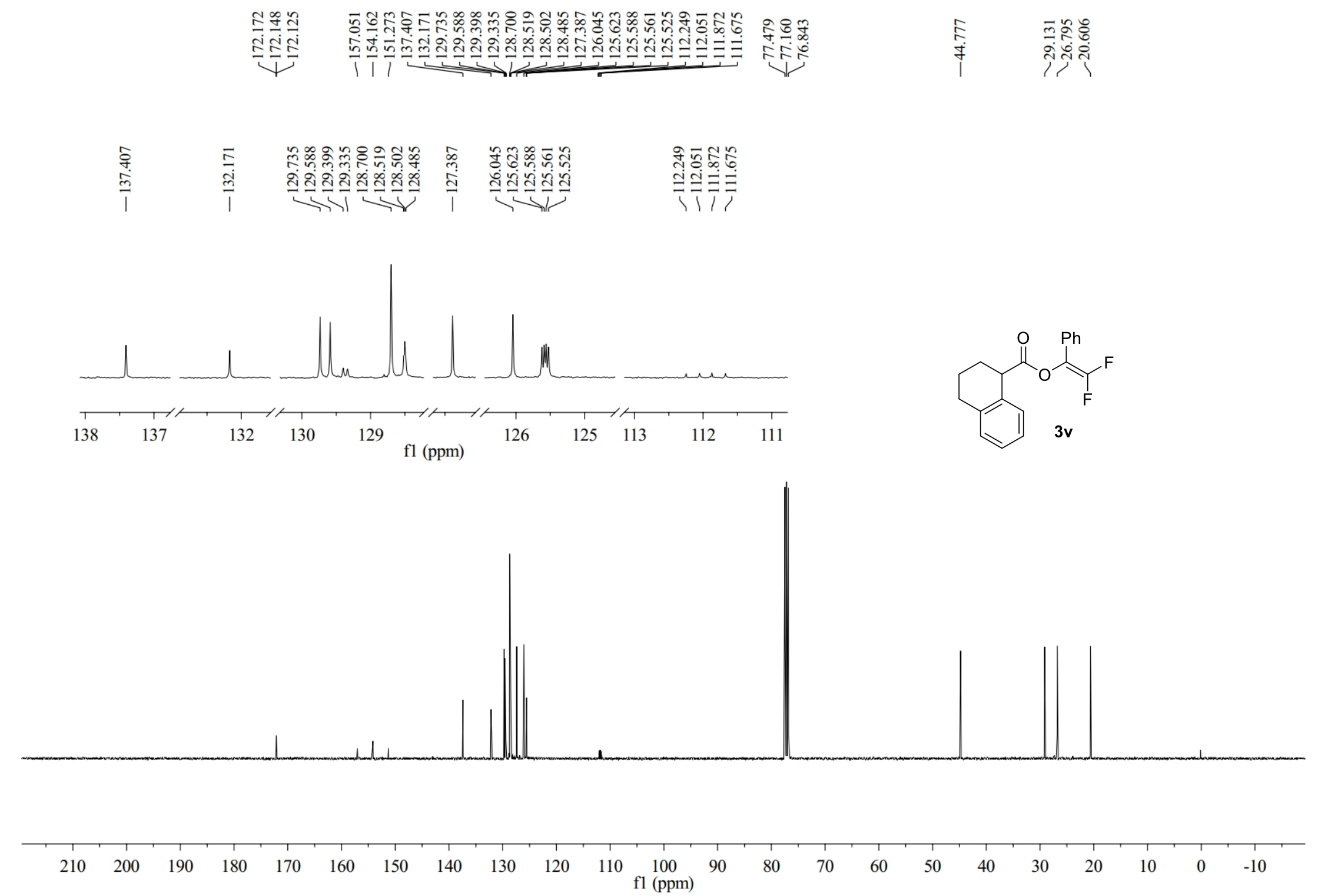

${ }^{13} \mathrm{C}$ NMR of Compound $3 \mathbf{v}\left(100 \mathrm{MHz}, \mathrm{CDCl}_{3}\right)$ 
نे

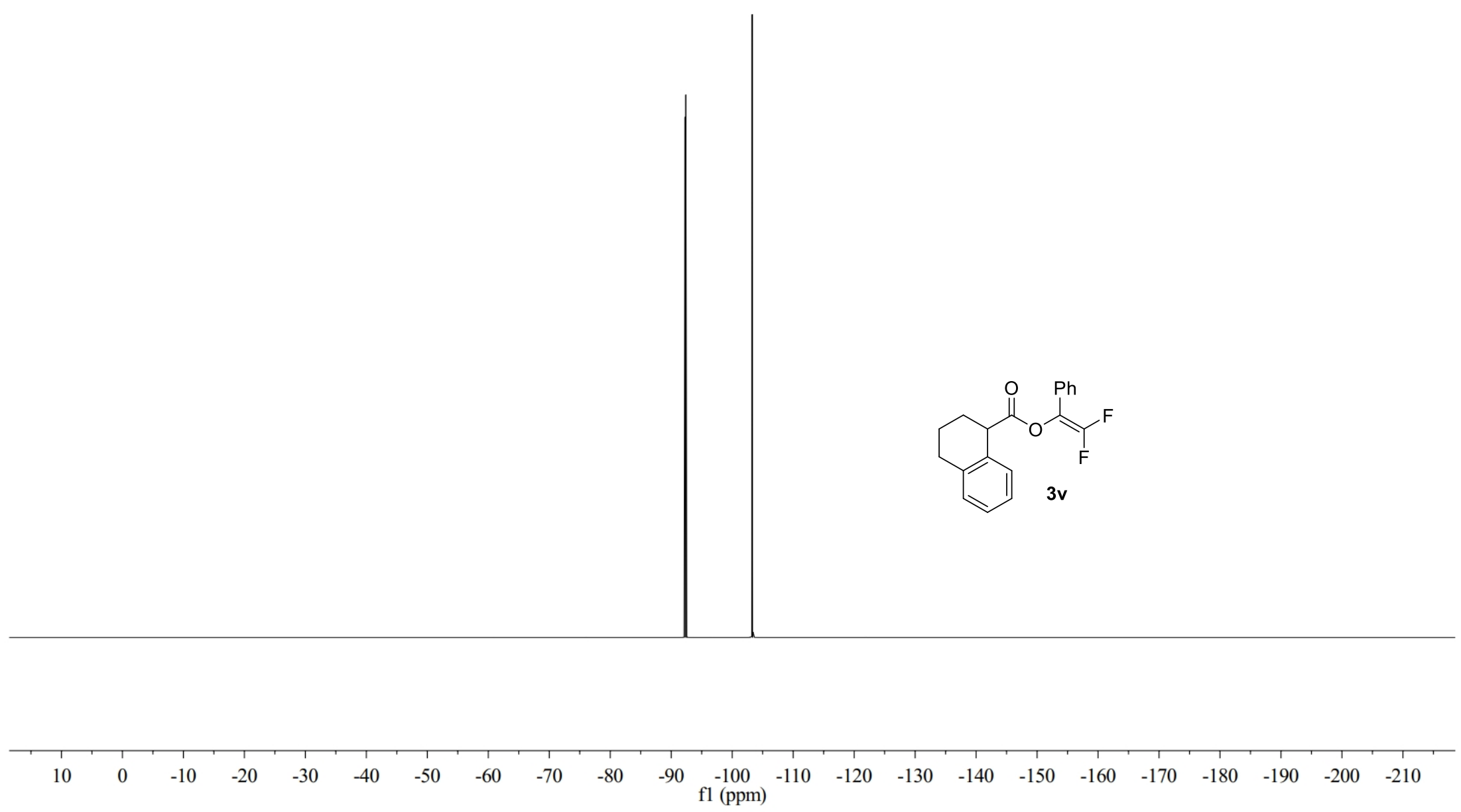

${ }^{19} \mathrm{~F}$ NMR of Compound $3 \mathbf{v}\left(376 \mathrm{MHz}, \mathrm{CDCl}_{3}\right)$ 


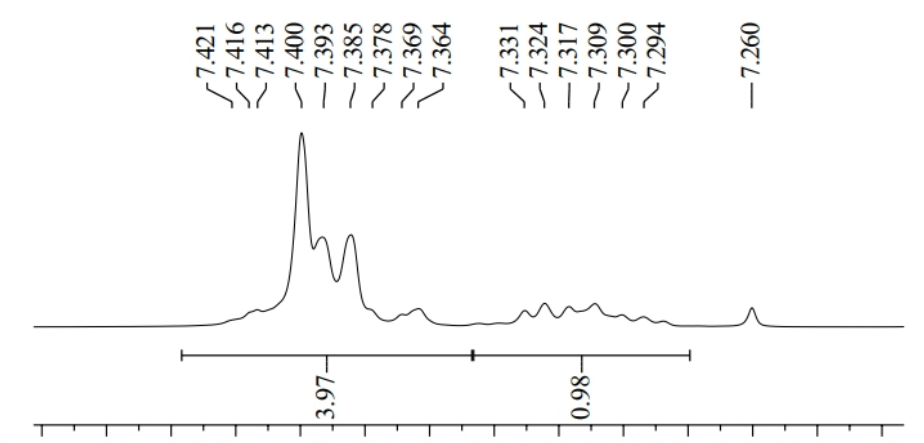

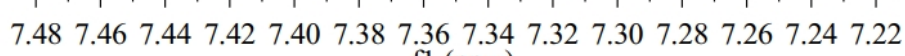
f1 (ppm)
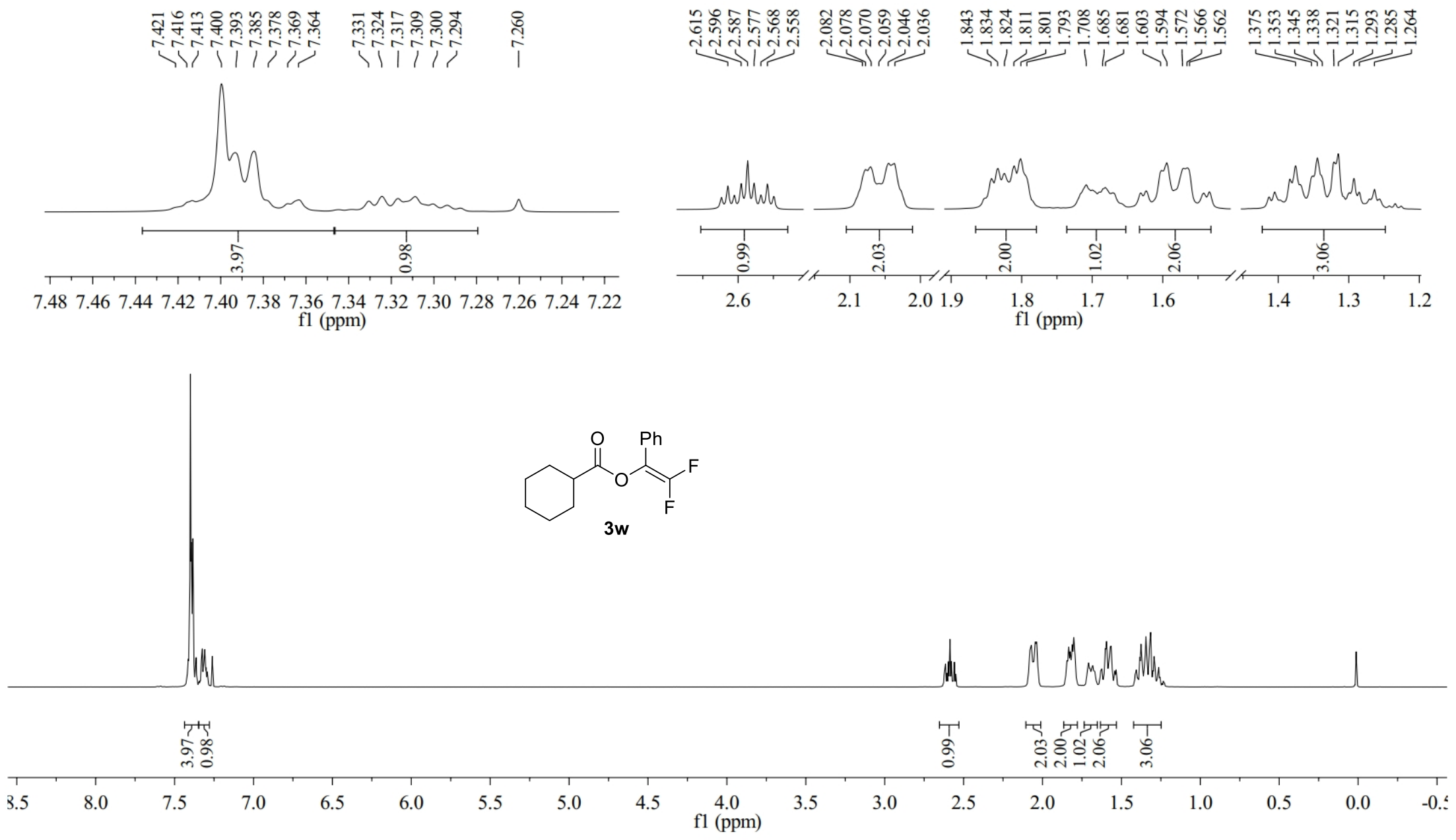

${ }^{1} \mathrm{H}$ NMR of Compound $\mathbf{3 w}\left(400 \mathrm{MHz}, \mathrm{CDCl}_{3}\right)$ 


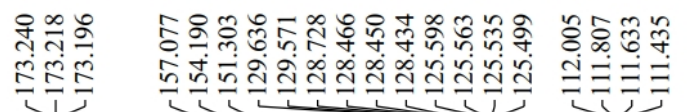

13

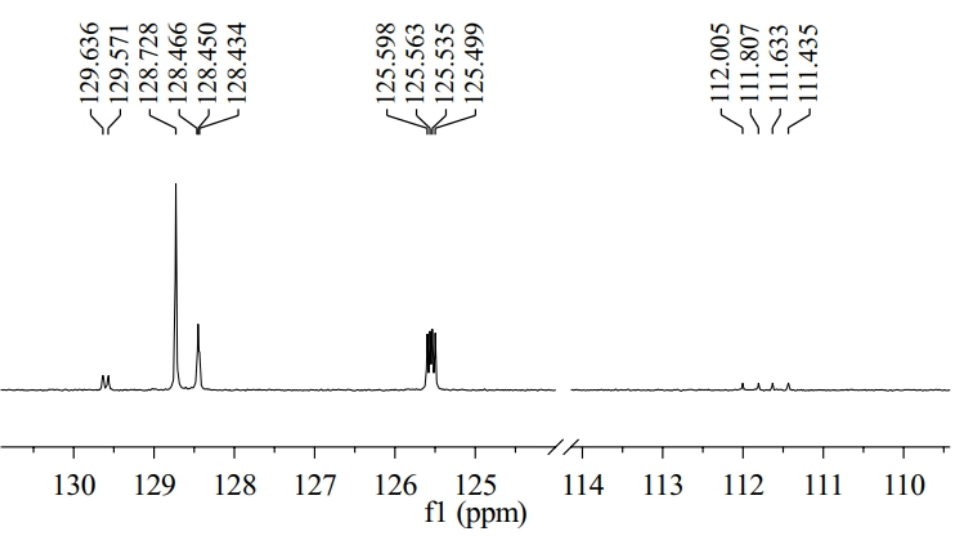

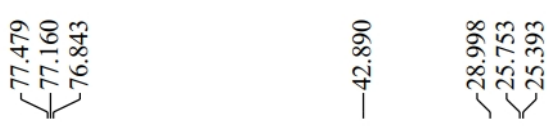

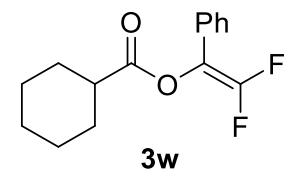

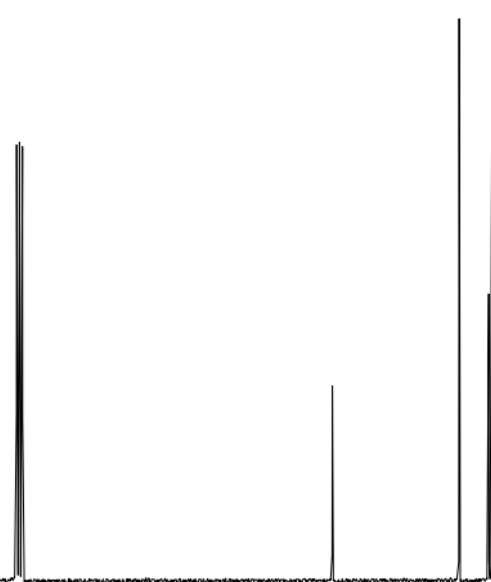

$\begin{array}{lllllllllllllllllllllllllllll}210 & 200 & 190 & 180 & 170 & 160 & 150 & 140 & 130 & 120 & 110 & 100 & 90 & 80 & 70 & 60 & 50 & 40 & 30 & 20 & 10 & 0 & -10 & \end{array}$

${ }^{13} \mathrm{C}$ NMR of Compound $3 \mathbf{w}\left(100 \mathrm{MHz}, \mathrm{CDCl}_{3}\right)$ 


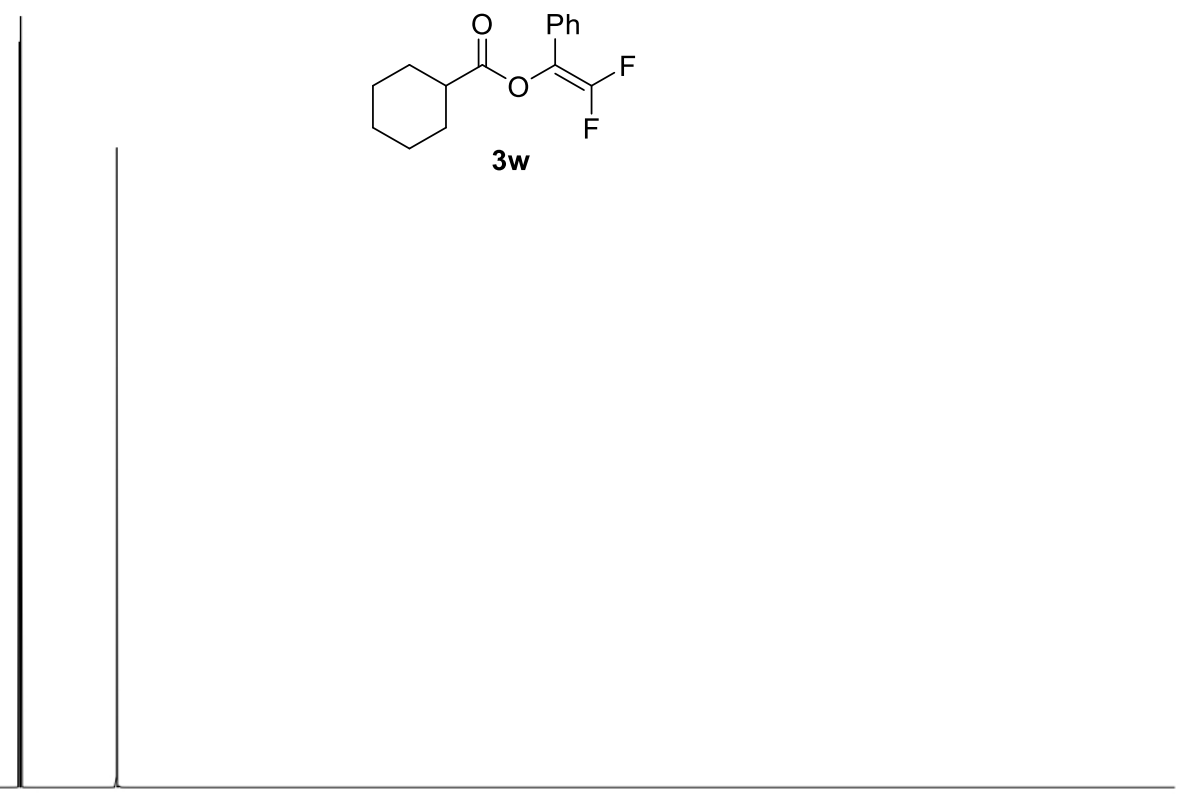

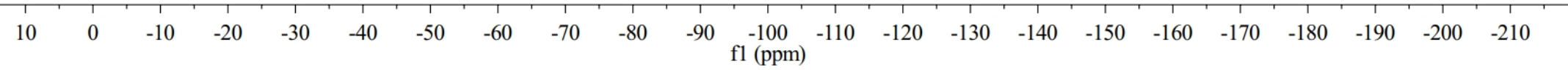

${ }^{19} \mathrm{~F}$ NMR of Compound 3w $\left(376 \mathrm{MHz}, \mathrm{CDCl}_{3}\right)$ 

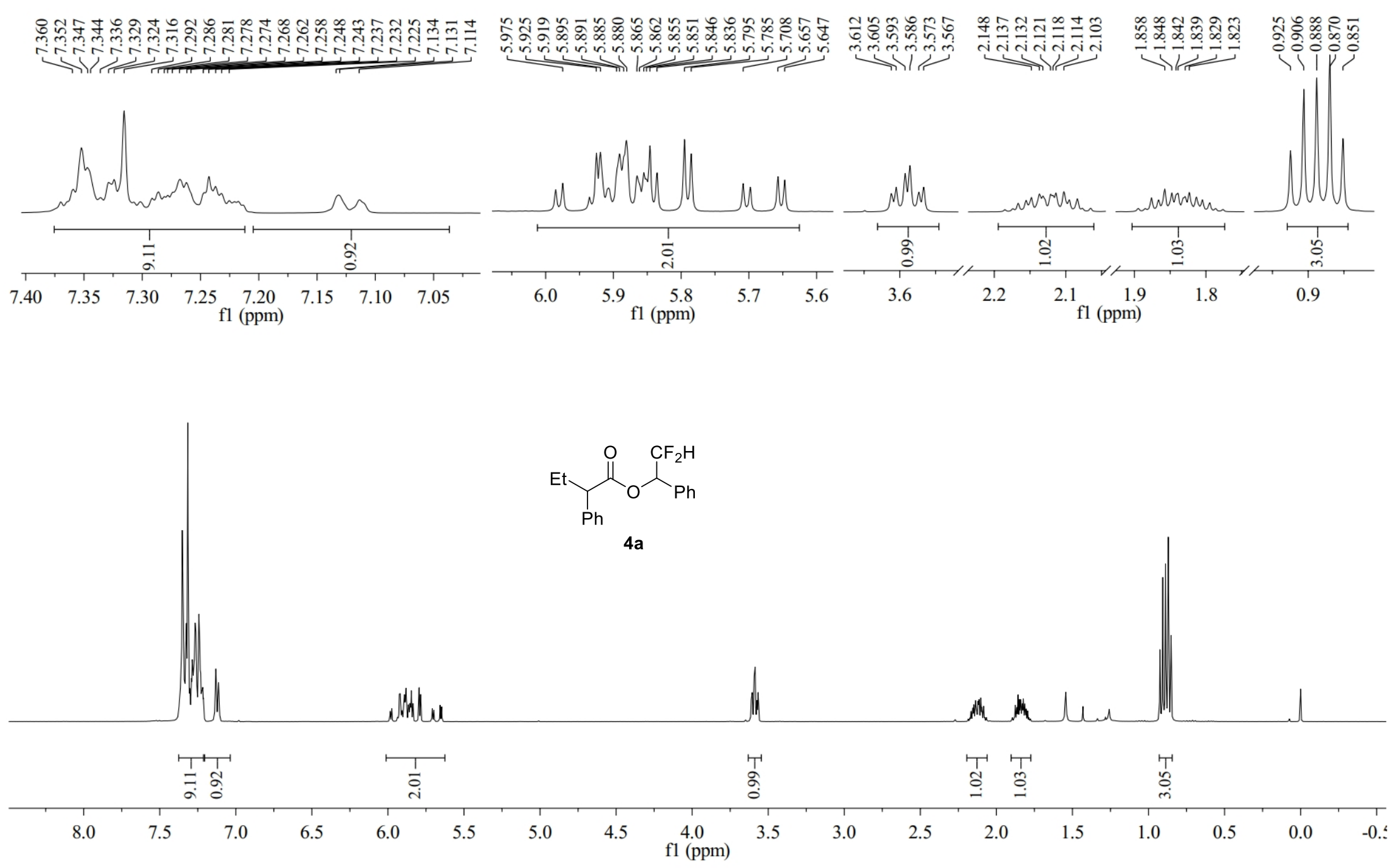

${ }^{1} \mathrm{H}$ NMR of Compound $\mathbf{4 a}\left(400 \mathrm{MHz}, \mathrm{CDCl}_{3}\right)$ 


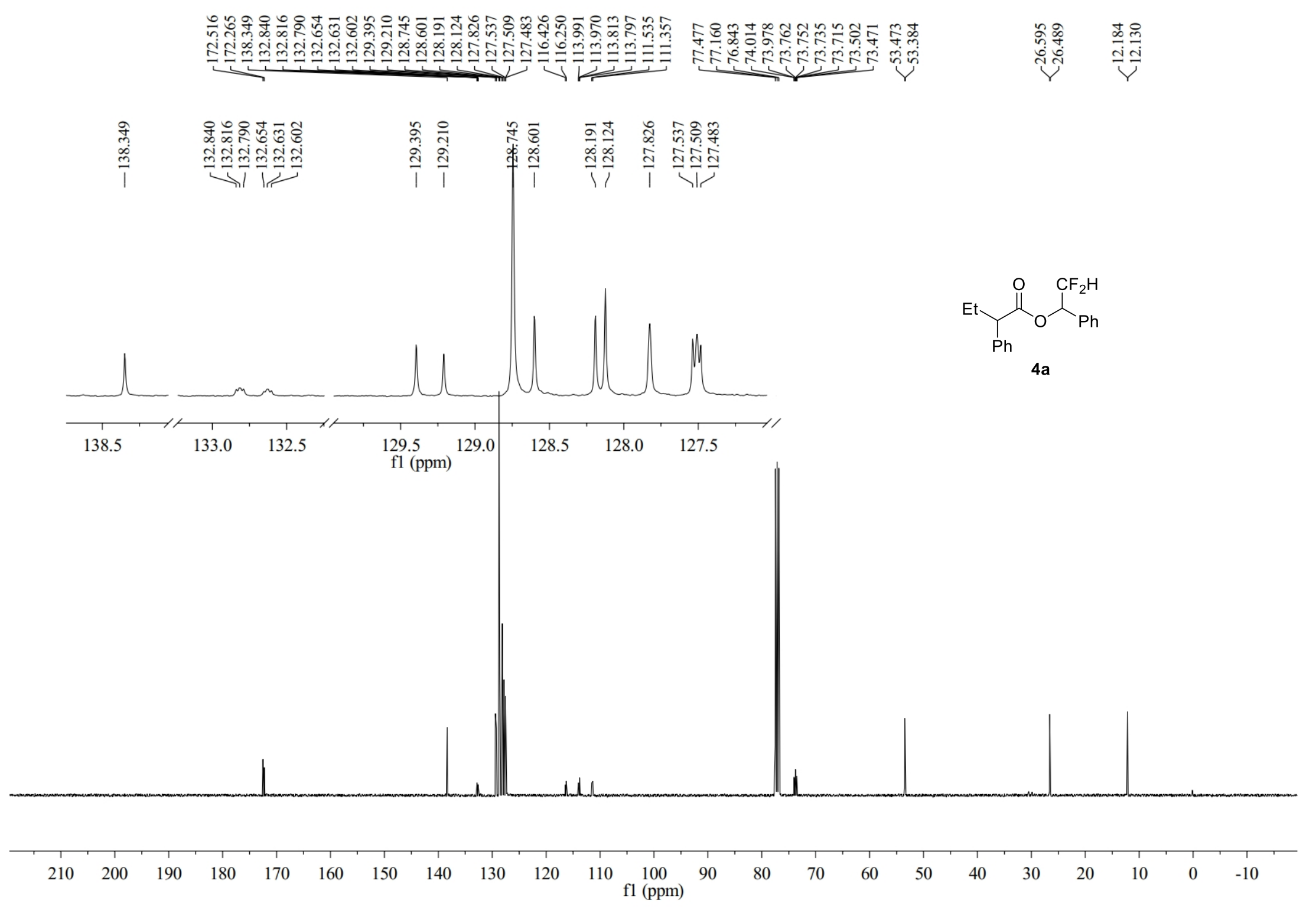

${ }^{13} \mathrm{C}$ NMR of Compound $\mathbf{4 a}\left(100 \mathrm{MHz}, \mathrm{CDCl}_{3}\right)$ 


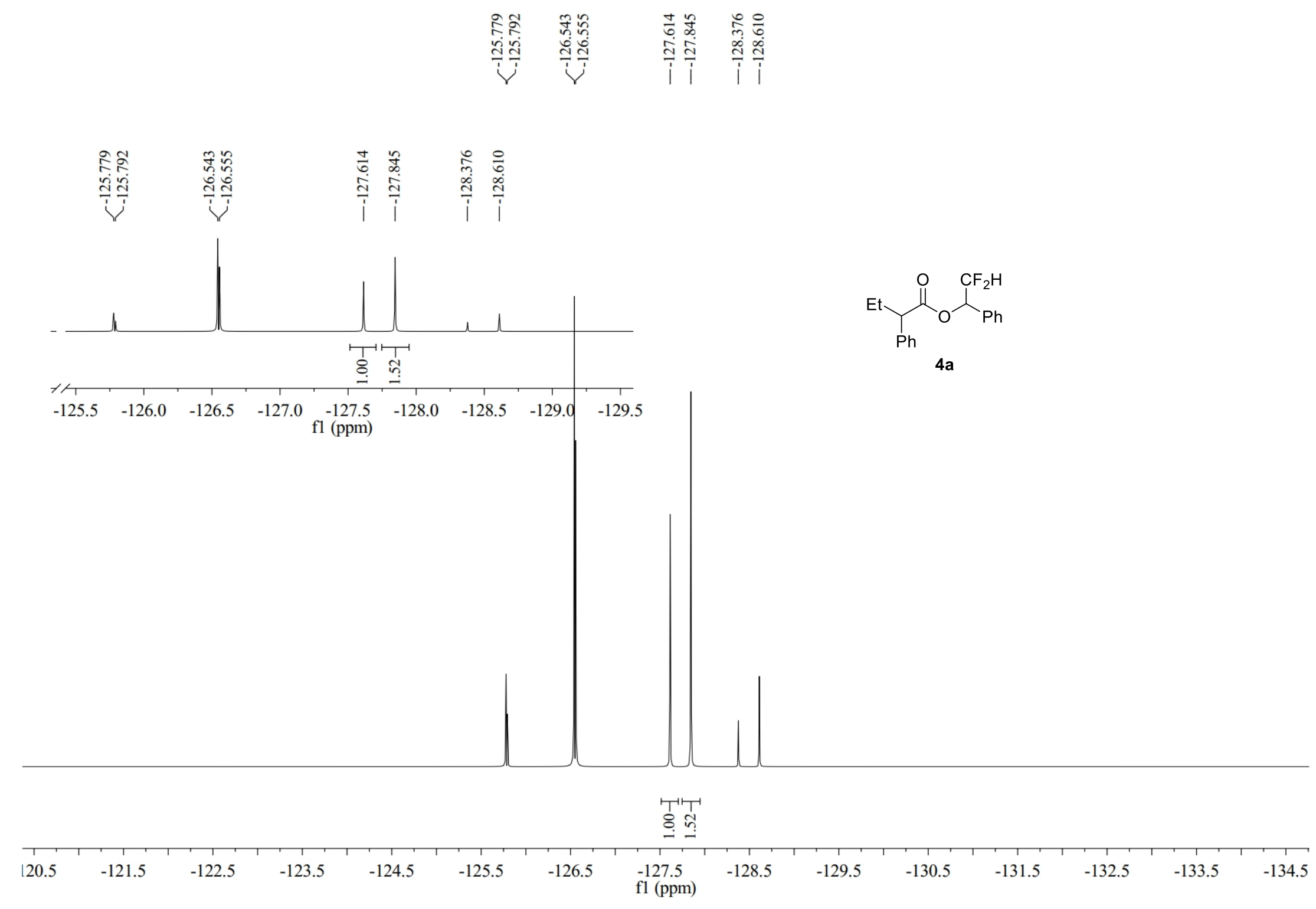

${ }^{19} \mathrm{~F}$ NMR of Compound $\mathbf{4 a}\left(376 \mathrm{MHz}, \mathrm{CDCl}_{3}\right)$ 


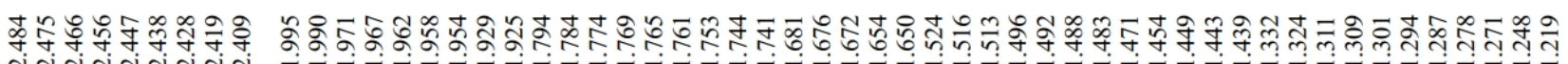

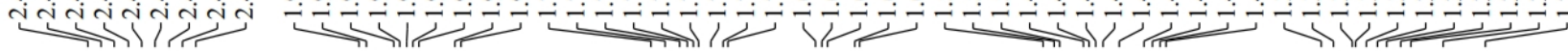

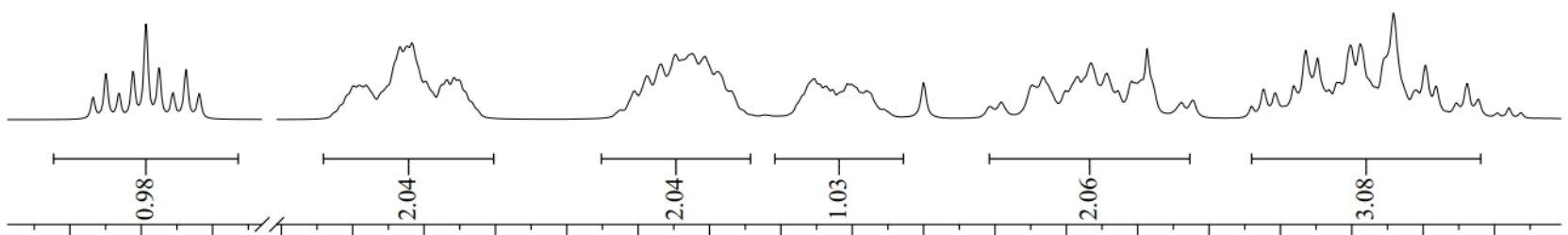

$\begin{array}{lllllllllllllllllllll}2.50 & 2.45 & 2.40 & 2.05 & 2.00 & 1.95 & 1.90 & 1.85 & 1.80 & 1.75 & 1.70 & 1.65 & 1.60 & 1.55 & 1.50 & 1.45 & 1.40 & 1.35 & 1.30 & 1.25 & 1.20\end{array}$

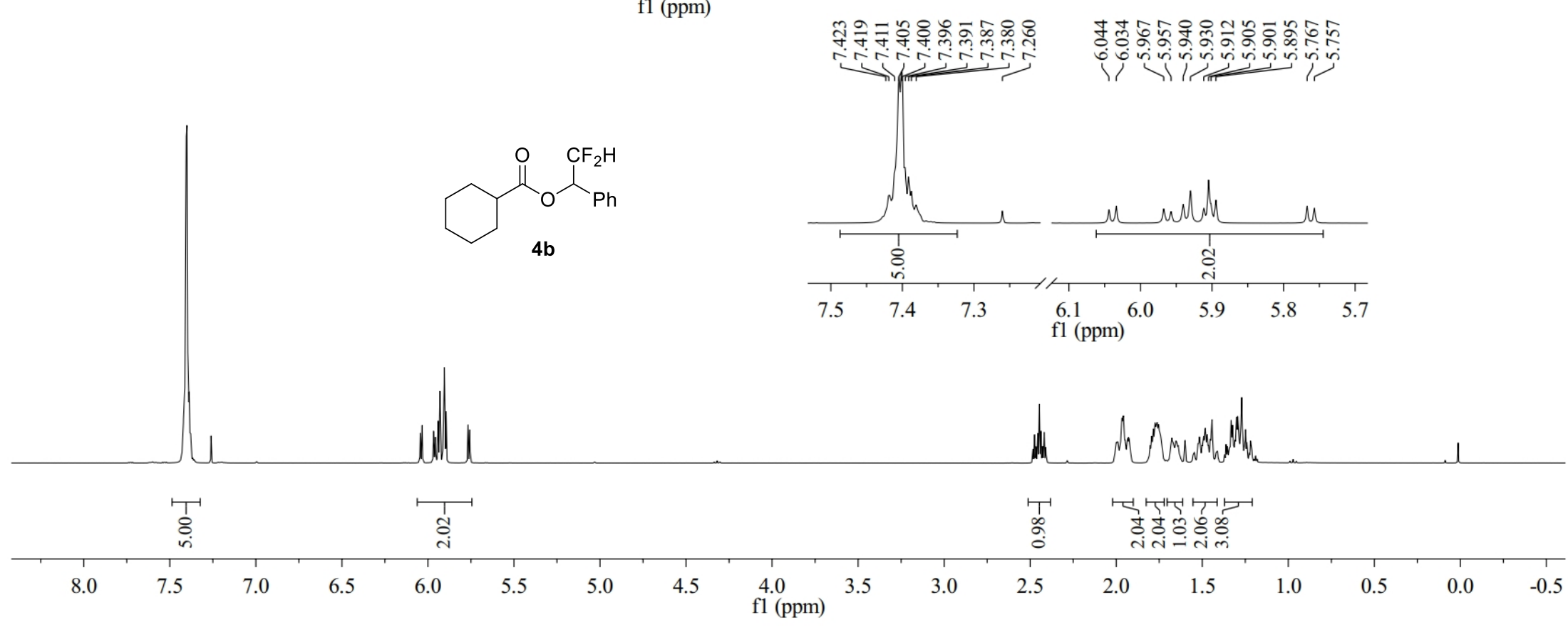

${ }^{1} \mathrm{H}$ NMR of Compound $\mathbf{4 b}\left(400 \mathrm{MHz}, \mathrm{CDCl}_{3}\right)$ 

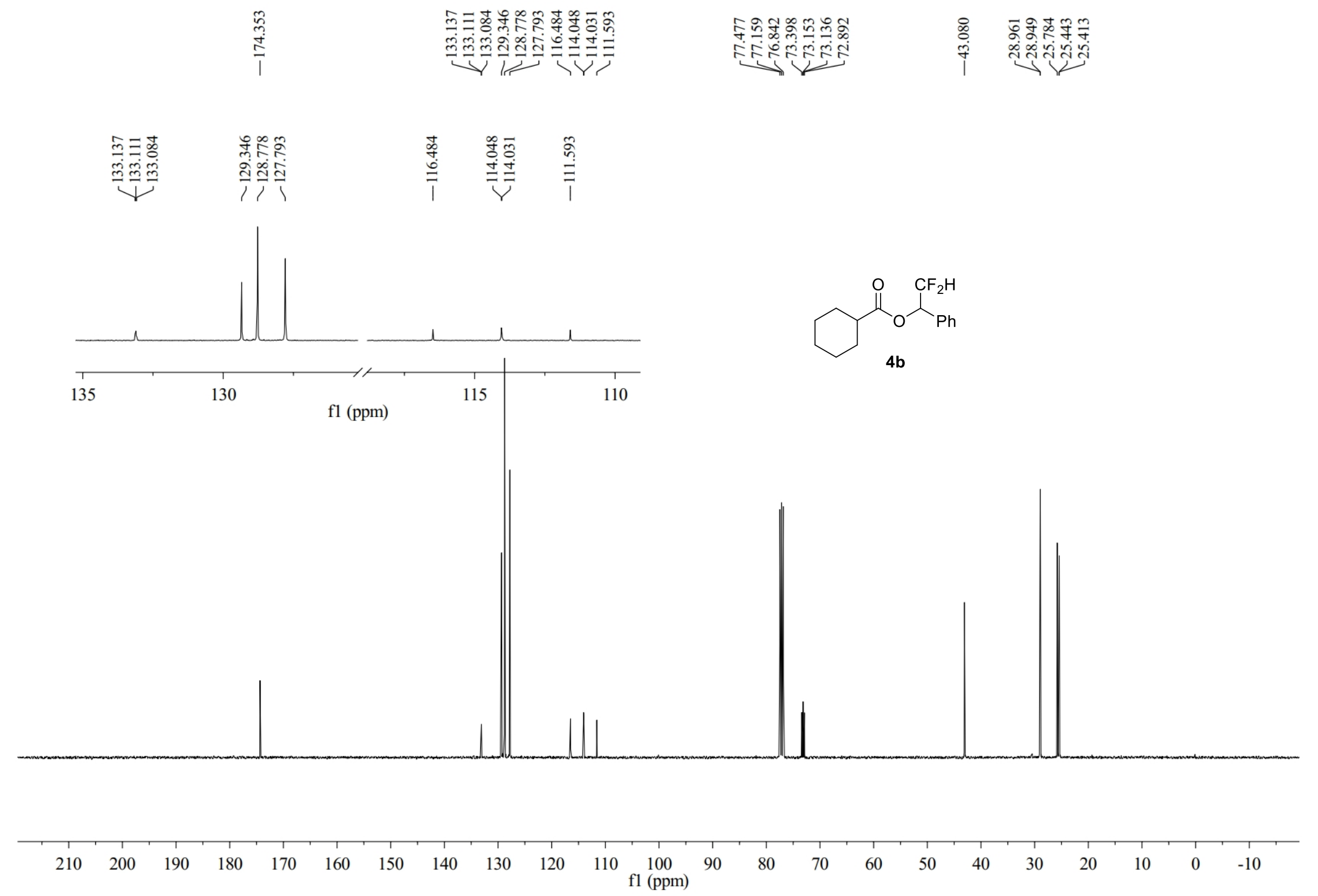

${ }^{13} \mathrm{C}$ NMR of Compound $\mathbf{4 b}\left(100 \mathrm{MHz}, \mathrm{CDCl}_{3}\right)$ 


$$
\text { 둥주ㅇㅝㅜ }
$$
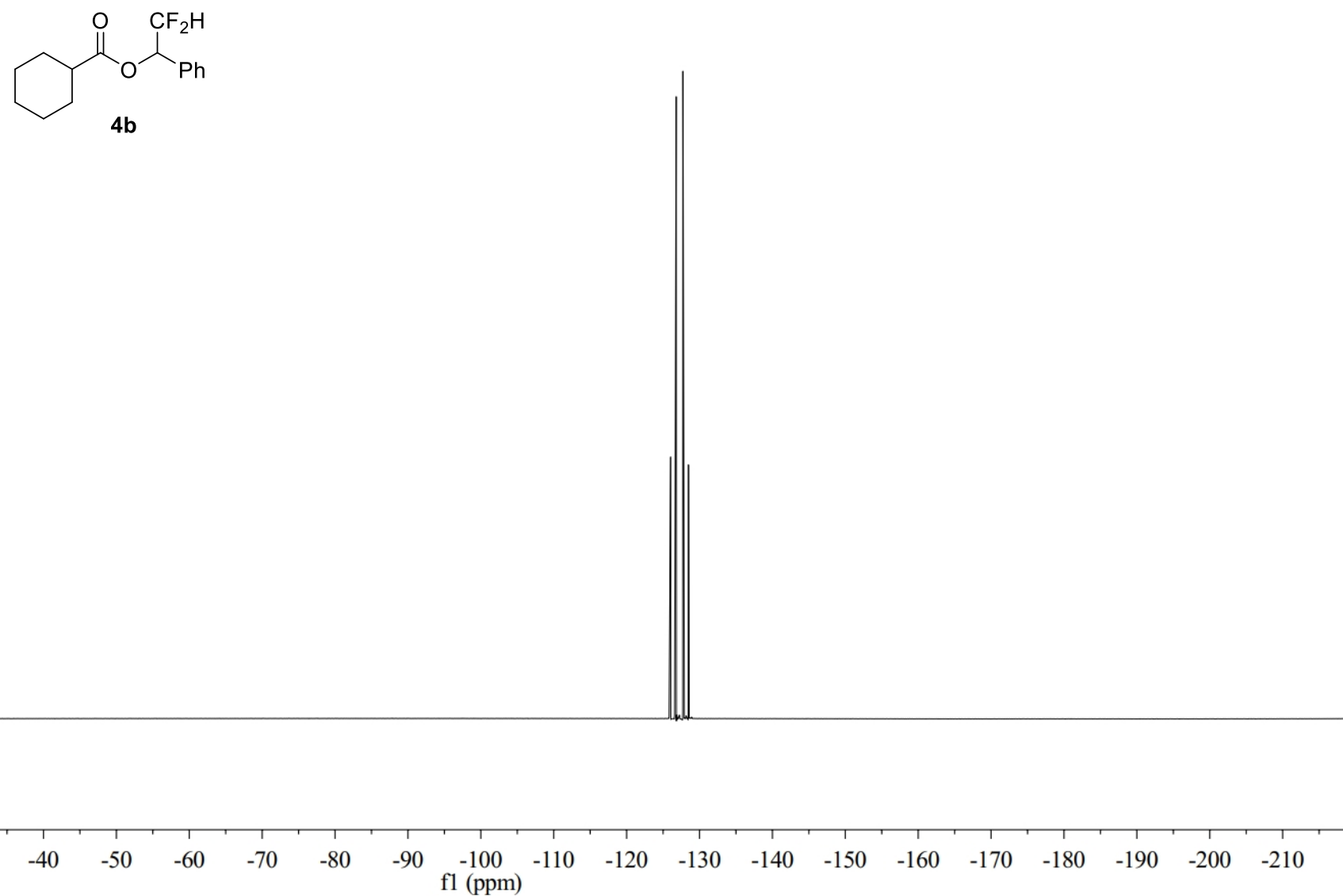

${ }^{19} \mathrm{~F}$ NMR of Compound $\mathbf{4 b}\left(376 \mathrm{MHz}, \mathrm{CDCl}_{3}\right)$ 

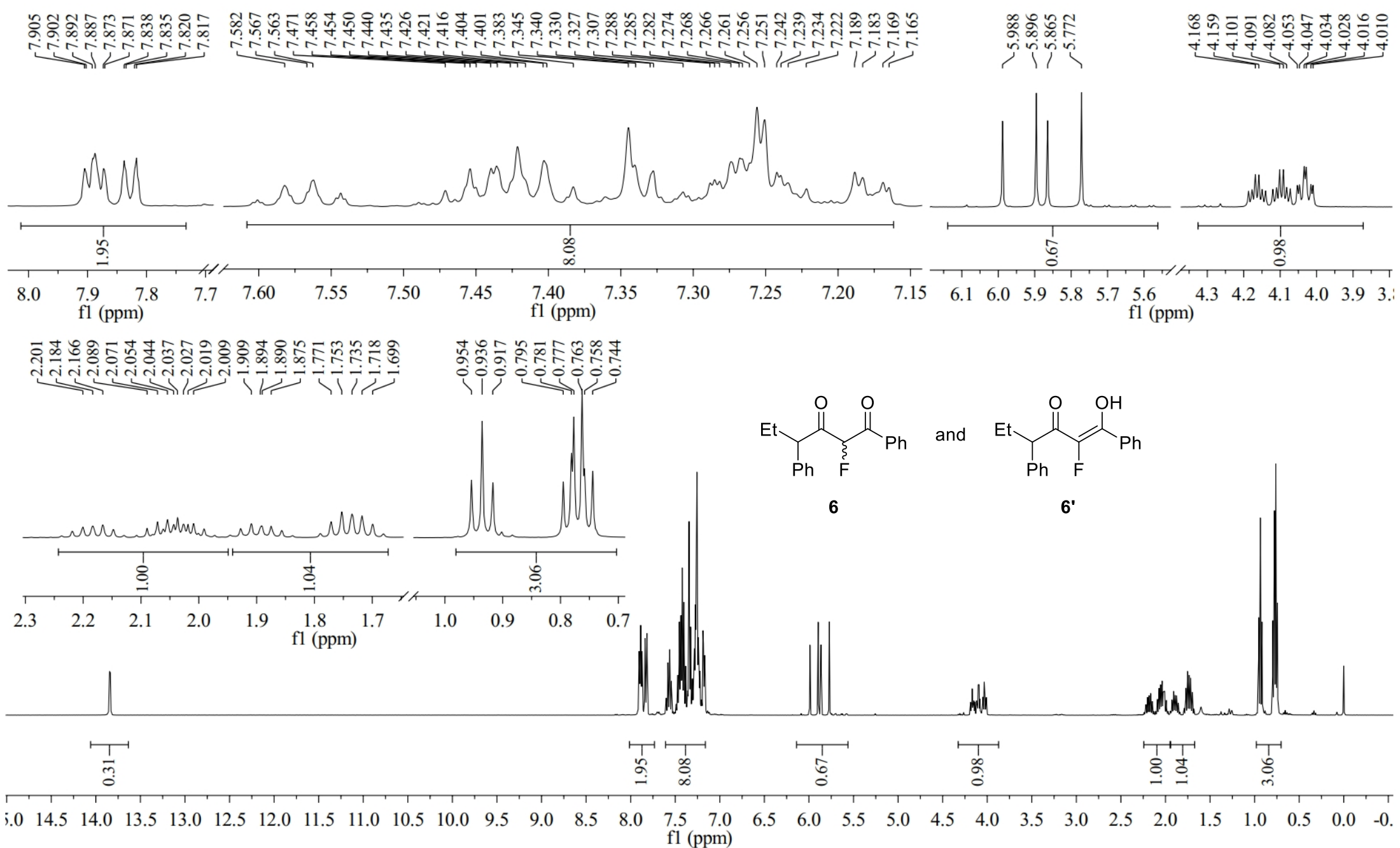

${ }^{1} \mathrm{H}$ NMR of Compound 6 and its enol derivative 6' $\left(400 \mathrm{MHz}, \mathrm{CDCl}_{3}\right)$ 


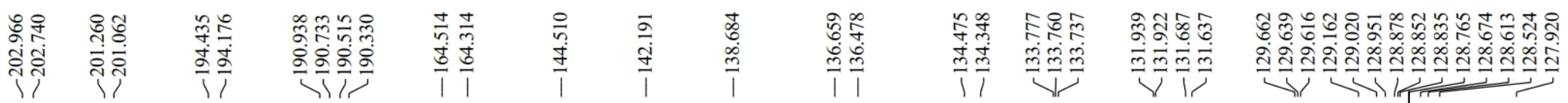

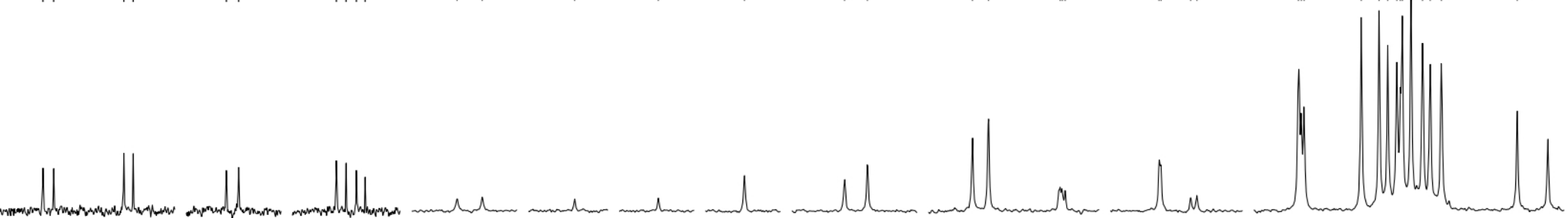

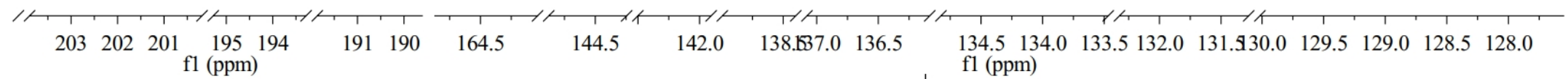

$\underbrace{H}_{P h}$ and

6

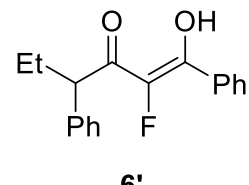

$\mathbf{6}^{\prime}$

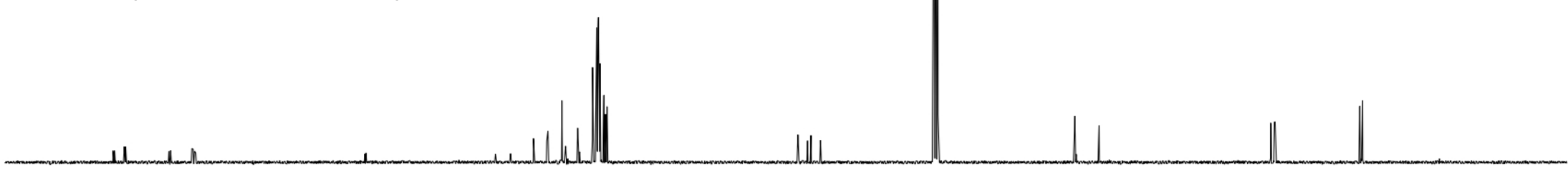

$\begin{array}{lllll}210 & 200 & 190 & 180 & 170\end{array}$

$160 \quad 150$

$140 \quad 130$

120

$110 \begin{aligned} & 100 \\ & \mathrm{fl}(\mathrm{ppm})\end{aligned}$

80

7060

50

40

$30 \quad 20$

10

$0 \quad-10$

${ }^{13} \mathrm{C}$ NMR of Compound 6 and its enol derivative 6' (100 $\mathrm{MHz}, \mathrm{CDCl}_{3}$ ) 

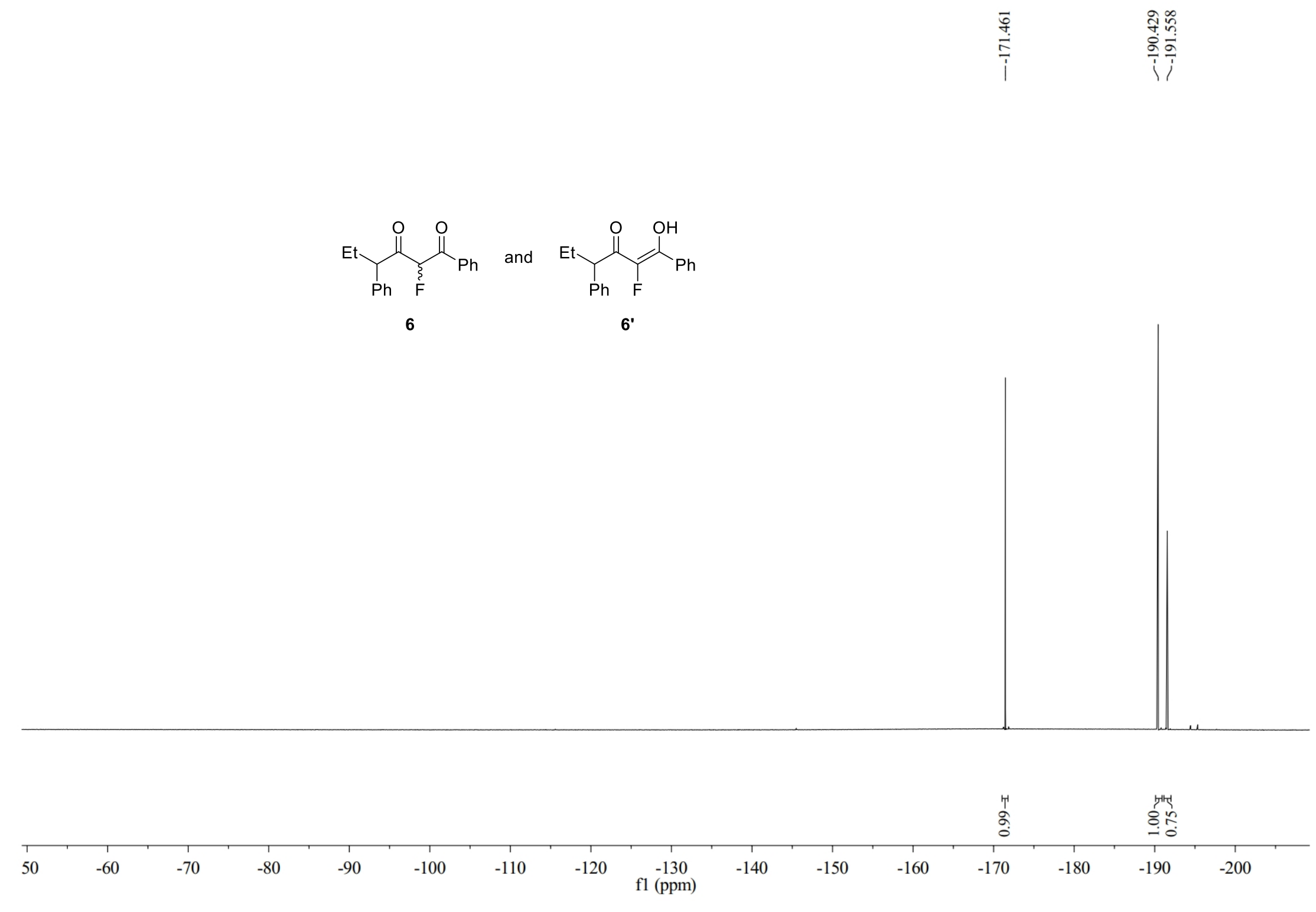

${ }^{19} \mathrm{~F}$ NMR of Compound 6 and its enol derivative 6' (376 MHz, $\left.\mathrm{CDCl}_{3}\right)$ 